\title{
Differentiable Stacks and Gerbes
}

\author{
Kai Behrend \\ University of British Columbia \\ 1984 Mathematics Road \\ Vancouver, B.C., Canada V6T $1 Z 2$ \\ behrend@math.ubc.ca \\ Ping $\mathrm{Xu}^{*}$ \\ Department of Mathematics \\ Pennsylvania State University \\ University Park, PA 16802 \\ ping@math.psu.edu
}

\begin{abstract}
We introduce differentiable stacks and explain the relationship with Lie groupoids. Then we study $S^{1}$-bundles and $S^{1}$-gerbes over differentiable stacks. In particular, we establish the relationship between $S^{1}$-gerbes and groupoid $S^{1}$-central extensions. We define connections and curvings for groupoid $S^{1}$-central extensions extending the corresponding notions of Brylinski, Hitchin and Murray for $S^{1}$-gerbes over manifolds. We develop a Chern-Weil theory of characteristic classes in this general setting by presenting a construction of Chern classes and Dixmier-Douady classes in terms of analogues of connections and curvatures. We also describe a prequantization result for both $S^{1}$-bundles and $S^{1}$-gerbes extending the well-known result of Weil and Kostant. In particular, we give an explicit construction of $S^{1}$-central extensions with prescribed curvature-like data.
\end{abstract}

\section{Contents}

1 Introduction 2

2 Differentiable Stacks 4

2.1 Groupoid fibrations . . . . . . . . . . . . . . . . . . . . 5

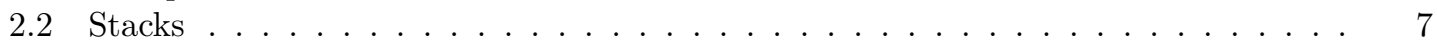

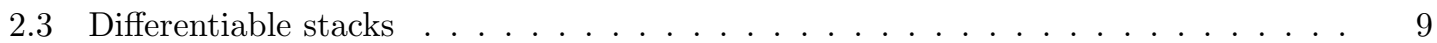

2.4 Torsors for Lie groupoids . . . . . . . . . . . . . . . . . . . . . 11

2.5 Morita equivalence . . . . . . . . . . . . . . . . . . . . . 14

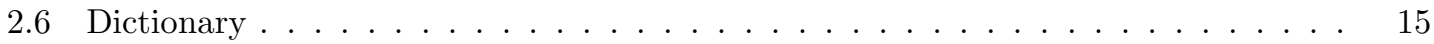

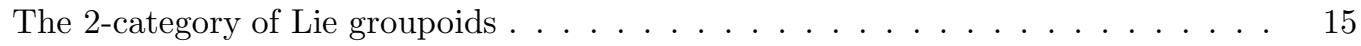

The Dictionary Lemmas . . . . . . . . . . . . . . . . . . . . . 15

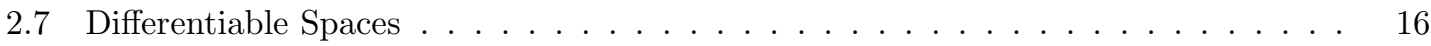

\footnotetext{
*Research partially supported by NSF grants DMS03-06665 and DMS-0605725 \& NSA grant 03G-142.
} 
3 Homology and cohomology 17

3.1 Sheaves over stacks and their cohomology . . . . . . . . . . . . . . . . . 18

3.2 Differential forms . . . . . . . . . . . . . . . . . . . . . . . . . 19

3.3 Groupoid cohomology . . . . . . . . . . . . . . . . . . . . 20

$4 \quad S^{1}$-bundles and $S^{1}$-gerbes $\quad \mathbf{2 2}$

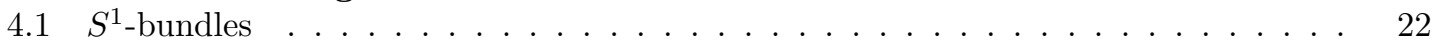

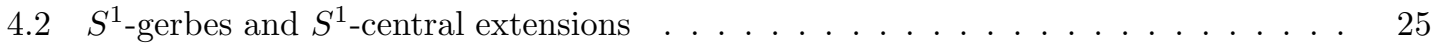

4.3 Morita equivalence of $S^{1}$-central extensions $\ldots \ldots \ldots \ldots \ldots \ldots$. . . . . . . 27

4.4 Dixmier-Douady classes . . . . . . . . . . . . . . . . . . . . . 29

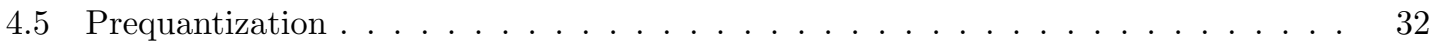

$5 \quad S^{1}$-central extensions with prescribed pseudo-curvature $\quad 34$

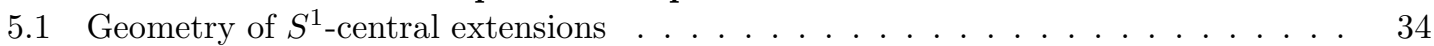

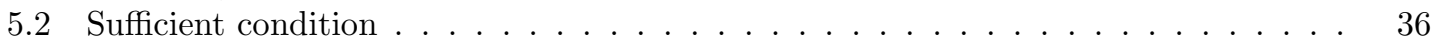

5.3 Properties of 3 -cocycles $\ldots \ldots \ldots \ldots \ldots \ldots$

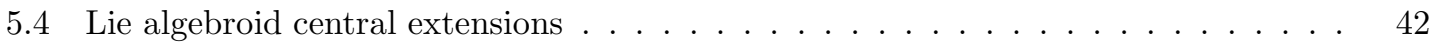

\section{Introduction}

Grothendieck introduced stacks to give geometric meaning to higher non-commutative cohomology classes. This is also the context in which gerbes first appeared [19]. However most of the work on stacks so far remains algebraic, though there is increasing evidence that differentiable stacks will find many useful applications. One example of the notion of stack is that of orbifolds. In algebraic geometry, these correspond to Deligne-Mumford stacks [25]. In differential geometry, orbifolds or $V$-manifolds have been studied for many years using local charts. Recently, it has been realized that viewing orbifolds as a very special kind of Lie groupoids, i.e. étale proper groupoids, is quite useful [35].

The notion of a groupoid is a generalization of the concepts of spaces and groups. A groupoid consists of a space of objects (units) $X_{0}$, and a space of arrows $X_{1}$ with source and target maps $s, t: X_{1} \rightarrow X_{0}$. There is a multiplication defined only for composable pairs $X_{2}=\{(x, y) \mid$ $t(x)=s(y)$, for $\left.x, y \in X_{1}\right\} \subset X_{1} \times X_{1}$. There is also an inverse map. These structures satisfy the usual axioms. Lie groupoids are groupoids where both $X_{0}$ and $X_{1}$ are manifolds, $s$ and $t$ are surjective submersions, and all the structure maps are required to be smooth. A Lie groupoid $X_{1} \rightrightarrows X_{0}$ is said to be proper if the map $s \times t: X_{1} \rightarrow X_{0} \times X_{0}$ is proper (in algebraic geometry, this would be called separated or Hausdorff). In the theory of groupoids, spaces and groups are treated on equal footing. Simplifying somewhat, one could say that a groupoid is a mixture of a space and a group; it has space-like and group-like properties that interact in a delicate way. In a certain sense, groupoids provide a uniform framework for many different geometric objects. For instance, when a Lie group acts on a manifold properly, the corresponding equivariant cohomology theories, including $K$-theory, can be treated using the transformation groupoid $M \rtimes G \rightrightarrows M$. Here the structure maps are $s(x, g)=x, t(x, g)=x g, \quad(x, g)(y, h)=(x, g h)$.

There exists a dictionary between differentiable stacks and Lie groupoids. Roughly speaking, differentiable stacks are Lie groupoids up to Morita equivalence. Any Lie groupoid $X_{1} \rightrightarrows X_{0}$ defines a differentiable stack $\mathfrak{X}$ of $X_{\bullet}$-torsors. Two differentiable stacks $\mathfrak{X}$ and $\mathfrak{X}^{\prime}$ are isomorphic if and only if the Lie groupoids $X \bullet$ and $X_{\bullet}^{\prime}$ are Morita equivalent. In a certain sense, Lie groupoids are like "local charts" on a differentiable stack. Establishing such a dictionary consists of the first part of the paper. We note that this viewpoint of connecting stacks with groupoids is somehow 
folklore (see $[15,34,39,40])$. However, we feel that it is useful to spell it out in detail in the differentiable geometry setting, which is of ultimate interest for our purpose.

Our main goal of this paper is to develop the theory of $S^{1}$-gerbes over differentiable stacks. Motivation comes from string theory in which "gerbes with connections" appear naturally $[13,16$, 23, 46].

For $S^{1}$-gerbes over manifolds, there has been extensive work on this subject pioneered by Brylinski [5], Chatterjee [8], Hitchin [21], Murray [32] and many others. Also, there is interesting work on equivariant $S^{1}$-gerbes, e.g., by Brylinski [6], Meinrenken [29], Gawedzki-Neis [17], Stienon [41] and others, as well as on gerbes over orbifolds [27]. These endeavors make the foundations of gerbes over differentiable stacks a very important subject. An important step is to geometrically realize a class $H^{2}\left(\mathfrak{X}, S^{1}\right)$ (or $H^{3}(\mathfrak{X}, \mathbb{Z})$ when $\mathfrak{X}$ is Hausdorff). Such a geometrical realization is crucial in applications to twisted $K$-theory $[43,44,45]$.

Our method is to use the dictionary mentioned above, under which we show that $S^{1}$-gerbes are in one-to-one correspondence with Morita equivalence classes of groupoid $S^{1}$-central extensions. Thus it follows from a well-known theorem of Giraud [19] that there is a bijection between $H^{2}\left(\mathfrak{X}, S^{1}\right)$ and Morita equivalence classes of Lie groupoid $S^{1}$-central extensions. We note that there are several independent investigations of similar topics; see [7, 36, 37, 42, 50].

An $S^{1}$-central extension of a Lie groupoid $X_{1} \rightrightarrows X_{0}$ is a Lie groupoid $R_{1} \rightrightarrows X_{0}$ with a groupoid morphism $\pi: R_{1} \rightarrow X_{1}$ such that ker $\pi \cong X_{0} \times S^{1}$ lies in the center of $R_{1}$. It is easy to see that $\pi: R_{1} \rightarrow X_{1}$ is then naturally an $S^{1}$-principal bundle. A standard example is an $S^{1}$-central extension of a Čech groupoid: Let $N$ be a manifold and $\alpha \in H^{3}(N, \mathbb{Z})$, and let $\left\{U_{i}\right\}$ be a good covering of $N$. Then the groupoid $\coprod_{i j} U_{i j} \rightrightarrows \coprod_{i} U_{i}$, where $U_{i j}=U_{i} \cap U_{j}$, which is called the Čech groupoid, is Morita equivalent to the manifold $N$. Then the $S^{1}$-gerbe corresponding to the class $\alpha$ can be realized as an $S^{1}$-central extension of groupoids $\coprod_{i j} U_{i j} \times S^{1} \rightarrow \coprod_{i j} U_{i j} \rightrightarrows \coprod_{i} U_{i}$, where the multiplication on $\coprod_{i j} U_{i j} \times S^{1}$ is given by $\left(x_{i j}, \lambda_{1}\right)\left(x_{j k}, \lambda_{2}\right)=\left(x_{i k}, \lambda_{1} \lambda_{2} c_{i j k}\right)$, where $x_{i j}, x_{j k}, x_{i k}$ are the same point $x$ in the three-intersection $U_{i j k}$ considered as elements in the two-intersections, and $c_{i j k}: U_{i j k} \rightarrow S^{1}$ is a 2-cocycle which represents the Čech class in $H^{2}\left(N, S^{1}\right) \cong H^{3}(N, \mathbb{Z})$ corresponding to $\alpha$. This is essentially the picture of an $S^{1}$-gerbe over a manifold described by Hitchin [21].

The exponential sequence $\mathbb{Z} \rightarrow \Omega^{0} \rightarrow S^{1}$ induces a boundary map $H^{2}\left(X_{\bullet}, S^{1}\right) \rightarrow H^{3}\left(X_{\bullet}, \mathbb{Z}\right)$. The image in $H^{3}\left(X_{\bullet}, \mathbb{Z}\right)$ of the class in $H^{2}\left(X_{\bullet}, S^{1}\right)$ of a groupoid $S^{1}$-central extension $R_{\bullet}$ is called the Dixmier-Douady class of $R_{\bullet}$. The Dixmier-Douady class behaves well with respect to the pullback and the tensor operations. A fundamental question is to develop a Chern-Weil characteristic class theory to construct the Dixmier-Douady classes geometrically. For this purpose, we need the de Rham double complex of a Lie groupoid. Let $X_{1} \rightrightarrows X_{0}$ be a Lie groupoid. By $X_{p}$, we denote the manifold of composable sequences of $p$ arrows in the groupoid $X_{1} \rightrightarrows X_{0}$. We have $p+1$ canonical maps, called face maps, $X_{p} \rightarrow X_{p-1}$ giving rise to a diagram

$$
\ldots X_{2} \Longrightarrow X_{1} \Longrightarrow X_{0} \text {. }
$$

In fact, $X$. is a simplicial manifold [14]. Thus for any abelian sheaf $F$ (e.g., $\mathbb{Z}, \mathbb{R}$, or $S^{1}$ ), we have the cohomology groups $H^{k}\left(X_{\bullet}, F\right)$. Just like for manifolds, $H^{k}(\mathfrak{X}, \mathbb{R})$ is canonically isomorphic to the de Rham cohomology of $X_{1} \rightrightarrows X_{0}$, which is defined by the double complex $\Omega^{\bullet}\left(X_{\bullet}\right)$, with boundary maps $d: \Omega^{k}\left(X_{p}\right) \rightarrow \Omega^{k+1}\left(X_{p}\right)$, the usual exterior derivative of differentiable forms, and $\partial: \Omega^{k}\left(X_{p}\right) \rightarrow \Omega^{k}\left(X_{p+1}\right)$, the alternating sum of the pull-back of face maps. We denote the total differential by $\delta=(-1)^{p} d+\partial$. The cohomology groups of the total complex $H_{d R}^{k}\left(X_{\bullet}\right)=$ $H^{k}\left(\Omega^{\bullet}\left(X_{\bullet}\right)\right)$ are called the de Rham cohomology groups of $X_{1} \rightrightarrows X_{0}$. When $X_{1} \rightrightarrows X_{0}$ is the Cech groupoid associated to an open covering of a manifold $N$, this is isomorphic to the usual de Rham cohomology of the manifold $N$. On the other hand, when $X_{1} \rightrightarrows X_{0}$ is a transformation groupoid $G \rtimes M \rightrightarrows M$, then $H_{d R}^{k}\left(X_{\bullet}\right)$ is isomorphic to the equivariant cohomology $H_{G}^{k}(M)$. 
Following Murray [32] and Hitchin [21], for a given groupoid $S^{1}$-central extension, one can also define the notions of connections, curvings and 3-curvatures. A flat gerbe is one whose 3curvature vanishes. In this case, there exists a holonomy map as well. However, a substantial difference between $S^{1}$-gerbes over an arbitrary differential stack and $S^{1}$-gerbes over a manifold is that connections and curvings may not always exist. Therefore they may not be as useful as one expects in calculating Dixmier-Douady classes. For this purpose, we need the notion of so called pseudo-connections. Given an $S^{1}$-central extension $R_{1} \rightarrow X_{1} \rightrightarrows X_{0}$, a pseudo-connection consists of a pair $(\theta, B)$, where $\theta \in \Omega^{1}\left(R_{1}\right)$ is a connection 1-form for the $S^{1}$-principal bundle $R_{1} \rightarrow X_{1}$, and $B \in \Omega^{2}\left(X_{0}\right)$ is a 2 -form. It is simple to check that $\delta(\theta+B) \in Z_{d R}^{3}\left(R_{\bullet}\right)$ descends to $Z_{d R}^{3}\left(X_{\bullet}\right)$, i.e. there exist unique $\eta \in \Omega^{1}\left(X_{2}\right), \omega \in \Omega^{2}\left(X_{1}\right)$ and $\Omega \in \Omega^{3}\left(X_{0}\right)$ such that $\delta(\theta+B)=\pi^{*}(\eta+\omega+\Omega)$. Then $\eta+\omega+\Omega$ is called the pseudo-curvature of the pseudo-connection $\theta+B$. It is simple to check that the class $[\eta+\omega+\Omega] \in H_{d R}^{3}\left(X_{\bullet}\right)$ is independent of the choice of the pseudo-connection $\theta+B$. One of the main results of this paper is that $[\eta+\omega+\Omega]$ is indeed the Dixmier-Douady class, or more precisely, the image of the Dixmier-Douady class under the canonical homomorphism $H^{3}(\mathfrak{X}, \mathbb{Z}) \rightarrow H^{3}(\mathfrak{X}, \mathbb{R}) \cong H_{d R}^{3}\left(X_{\bullet}\right)$. Recently, Ginot-Stienon found an alternative proof of this result using 2-group bundles [18] (in fact they proved a more general result for central $G$-extensions). We also describe a prequantization result, an analogue of the Kostant-Weil $[24,48]$ theorem for $S^{1}$-gerbes. That is, given any integral 3-cocycle $\eta+\omega+\Omega \in Z_{d R}^{3}\left(X_{\bullet}\right)$, we describe a sufficient condition that guarantees the 3 -cocycle as the pseudo-curvature of a groupoid $S^{1}$-central extension $R_{\bullet}$ with a pseudo-connection $\theta+B$, and classify all such pairs $\left(R_{\bullet}, \theta+B\right)$.

$S^{1}$-central extensions of Lie groupoids also appear naturally in Poisson geometry. It was proved in [49] that a certain prequantization of a symplectic groupoid naturally becomes an $S^{1}$-central extension of groupoids with a connection, which is indeed a contact groupoid. The proof utilizes Lie algebroids as a tool. Lie algebroids are infinitesimal versions of Lie groupoids. It is thus natural to study Lie groupoid central extensions via Lie algebroid central extensions in a general framework. More precisely, let $X_{1} \rightrightarrows X_{0}$ be an $s$-connected Lie groupoid with Lie algebroid $A$, and let $\eta+\omega \in$ $Z_{d R}^{3}\left(X_{\bullet}\right)$ be a de-Rham 3-cocycle, where $\eta \in \Omega^{1}\left(X_{2}\right)$ and $\omega \in \Omega^{2}\left(X_{1}\right)$. Then $\omega-d \eta^{r} \in \Omega^{2}\left(X_{1}^{t}\right)$ is a right invariant $t$-fiberwise closed two-form on $X_{1}$, and therefore defines a Lie algebroid two-cocycle of $A$, which in turn defines a Lie algebroid central extension $\widetilde{A}=A \oplus\left(X_{0} \times \mathbb{R}\right)$ of $A$. Here $\eta^{r}$ is a $t$-fiberwise one-form on $X_{1}$ given by $\eta^{r}\left(\delta_{x}\right)=\eta\left(r_{x^{-1} *} \delta_{x}, 0_{x}\right), \forall \delta_{x} \in T_{x} X_{1}^{t}$, and $r_{x^{-1}}$ denotes the right translation. A natural question is: under what condition does this Lie algebroid central extension give rise to a Lie groupoid central extensione? The last part of the paper is devoted to investigating this question. Our method is to adapt the method of characteristics developed by Coste-Dazord-Weinstein [9]. As a consequence, we obtain a geometrical characterization of the integrality condition of a de Rham 3-cocycle $\eta+\omega \in Z_{d R}^{3}\left(X_{\bullet}\right)$ of a Lie groupoid $X_{1} \rightrightarrows X_{0}$.

The results of this paper were announced in [3]. See also [4] for a construction of $S^{1}$-gerbes over the quotient stack $[G / G]$ ( $G$ is a compact simple Lie group and $G$ acts on $G$ by conjugations) as an example.

Acknowledgments. We would like to thank several institutions for their hospitality while work on this project was being done: RIMS and IHP (Behrend and Xu), University of British Columbia and Université Pierre et Marie Curie (Xu). We also wish to thank many people for useful discussions and comments, including Camille Laurent-Gengoux, Jim Stasheff, Mathieu Stienon, Jean-Louis Tu and Alan Weinstein.

\section{Differentiable Stacks}

Our goal in this section is to define the notion of differentiable stack and establish a dictionary between differentiable stacks and Lie groupoids. Roughly speaking, differentiable stacks are Lie groupoids up to Morita equivalence. 
Our differentiable manifolds will not be assumed to necessarily be Hausdorff. We use the words $C^{\infty}$ and smooth interchangeably. The manifold consisting of one point is denoted by $*$ or $p t$.

Let us start by recalling some terminology. A $C^{\infty}$-map $f: U \rightarrow X$ of $C^{\infty}$ manifolds is a submersion, if for all $u \in U$ the derivative $f_{*}: T_{u} U \rightarrow T_{f(u)} X$ is surjective. The relative dimension of the submersion $f$ is the (locally constant on $U$ ) dimension of the kernel of $f_{*}$. A submersive map of relative dimension 0 is called étale. Thus $f$ is étale if and only if it is a local diffeomorphism.

Let $\mathfrak{S}$ be the category of all $C^{\infty}$-manifolds with $C^{\infty}$-maps as morphisms. Note that not all fiber products exist in $\mathfrak{S}$, but if at least one of the two morphisms $U \rightarrow X$ or $V \rightarrow X$ is submersive, then the fiber product $U \times_{X} V$ exists in $\mathfrak{S}$. In general, the fiber product $U \times_{X} V$ exists if $U \rightarrow X$ and $V \rightarrow X$ satisfy the transversality condition.

We endow $\mathfrak{S}$ with the Grothendieck topology given by the following notion of covering family. Call a family $\left\{U_{i} \rightarrow X\right\}$ of morphisms in $\mathfrak{S}$ with target $X$ a covering family of $X$, if all maps $U_{i} \rightarrow X$ are étale and the total map $\coprod_{i} U_{i} \rightarrow X$ is surjective.

One checks that the conditions for a Grothendieck topology (see Exposé II in [1]) are satisfied. (Note that in the terminology of loc. cit. we have actually defined a pretopology. This pretopology gives rise to a Grothendieck topology, as explained in loc. cit..) We call this topology the étale topology on $\mathfrak{S}$.

One can also work with the topology of open covers. In this topology, all covering families are open covers $\left\{U_{i} \rightarrow X\right\}$, in the usual topological sense. The notion of sheaf or stack over $\mathfrak{S}$ obtained using this topology is the same as using the étale topology.

A site is just a category endowed with a Grothendieck topology. So if we refer to $\mathfrak{S}$ as a site, we emphasize that we think of $\mathfrak{S}$ together with its étale topology.

A Lie groupoid is a groupoid in $\mathfrak{S}$, whose source and target maps are submersions.

\subsection{Groupoid fibrations}

A category fibered in groupoids $\mathfrak{X} \rightarrow \mathfrak{S}$ is a category $\mathfrak{X}$, together with a functor $\pi: \mathfrak{X} \rightarrow \mathfrak{S}$, such that the following two fibration axioms are satisfied:

(i) for every arrow $V \rightarrow U$ in $\mathfrak{S}$, and every object $x$ of $\mathfrak{X}$ lying over $U$ (i.e. $\pi(x)=U$ ), there exists an arrow $y \rightarrow x$ in $\mathfrak{X}$ lying over $V \rightarrow U$,

(ii) for every commutative triangle $W \rightarrow V \rightarrow U$ in $\mathfrak{S}$ and arrows $z \rightarrow x$ lying over $W \rightarrow U$ and $y \rightarrow x$ lying over $V \rightarrow U$, there exists a unique arrow $z \rightarrow y$ lying over $W \rightarrow V$, such that the composition $z \rightarrow y \rightarrow x$ equals $z \rightarrow x$.

The object $y$ over $V$, whose existence is asserted in (i), is unique up to a unique isomorphism by (ii). Any choice of such a $y$ is called a pullback of $x$ via $V \rightarrow U$, notation $y=x \mid V$, or $y=f^{*} x$, if the morphism $V \rightarrow U$ is called $f$. Often it is convenient to choose pullbacks for all $x$ and all $V \rightarrow U$ (where $U=\pi(x))$.

Given a category fibered in groupoids $\mathfrak{X} \rightarrow \mathfrak{S}$ and an object $U$ of $\mathfrak{S}$, the category of all objects of $\mathfrak{X}$ lying over $U$ and all morphisms of $\mathfrak{X}$ lying over $\operatorname{id}_{U}$ is called the fiber of $\mathfrak{X}$ over $U$, notation $\mathfrak{X}_{U}$, sometimes $\mathfrak{X}(U)$. Note that all fibers $\mathfrak{X}_{U}$ are (set-theoretic) groupoids. This follows from Property (ii), above.

We call categories fibered in groupoids over $\mathfrak{S}$ also simply groupoid fibrations. The groupoid fibrations over $\mathfrak{S}$ (see [20]) form a 2-category. Fibered products exist. They satisfy a 2-categorical version of the universal mapping property for fibered products (see [25]).

The notion of groupoid fibration is the mathematical formalization of the notion of moduli problem. Let $\mathfrak{X} \rightarrow \mathfrak{S}$ be a groupoid fibration. If we consider $\mathfrak{X}$ as a moduli problem, then we think of an object $x \in \mathfrak{X}$ lying over $S \in \mathfrak{S}$ as an $\mathfrak{X}$-family parametrized by $S$. The objects we wish to classify are the objects of the category $\mathfrak{X}(p t)$.

Standard examples of categories fibered in groupoids over $\mathfrak{S}$ are: 
Example 2.1 Let $G$ be a Lie group. Let $\mathfrak{X}=B G$ be the category of pairs $(S, P)$, where $S \in \mathfrak{S}$ is a $C^{\infty}$-manifold and $P$ is a principal $G$-bundle over $S$. A morphism from $(S, P)$ to $(T, Q)$ is a commutative diagram

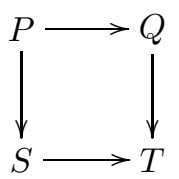

where $P \rightarrow Q$ is $G$-equivariant. The functor $\pi: B G \rightarrow \mathfrak{S}$ is defined by $(S, P) \mapsto S$.

Example 2.2 Every manifold $X$ defines a groupoid fibration $F_{X}$ over $\mathfrak{S}$. The objects of $F_{X}$ are pairs $(U, f)$, where $U$ is a $C^{\infty}$-manifold and $f: U \rightarrow X$ is a smooth map. Morphisms in $F_{X}$ are the commutative triangles

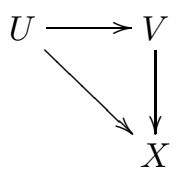

The functor $F_{X} \rightarrow \mathfrak{S}$ is the projection onto the first component. The groupoid fibration $F_{X}$ satisfies

$$
F_{X}(U)=\operatorname{Hom}_{\mathfrak{S}}(U, X) .
$$

By abuse of notation, we identify $F_{X}$ with $X$ in the sequel.

Example 2.3 Let $\mathfrak{M}_{g}$ be the following groupoid fibration: objects are fiber bundles $X \rightarrow S$ endowed with a smoothly varying fiberwise complex structure, such that all fibers are Riemann surfaces of genus $g$. Morphisms are commutative diagrams

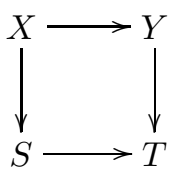

such that $X \rightarrow Y \times_{T} S$ is a conformal isomorphism. This groupoid fibration is the moduli stack of Riemann surfaces of genus $g$. An object $X \rightarrow S$ of $\mathfrak{M}_{g}$ is a family of Riemann surfaces parametrized by $S$. The functor $\mathfrak{M}_{g} \rightarrow \mathfrak{S}$ maps $X \rightarrow S$ to $S$.

Example 2.4 Any contravariant functor $F: \mathfrak{S} \rightarrow$ (sets) gives rise to a category fibered in groupoids $\mathfrak{X} \rightarrow \mathfrak{S}$ defined as follows: objects of $\mathfrak{X}$ are pairs $(U, x)$, where $U$ is a $C^{\infty}$-manifold and $x \in F(U)$. A morphism $(U, x) \rightarrow(V, y)$ is a $C^{\infty}$ map $a: U \rightarrow V$ such that $F(a)(y)=x$. The functor $\pi: \mathfrak{X} \rightarrow \mathfrak{S}$ is defined by $(U, x) \mapsto U$.

In particular, a sheaf over $\mathfrak{S}$ defines a groupoid fibration over $\mathfrak{S}$ in a canonical way.

Definition 2.5 A groupoid fibration $\mathfrak{X}$ over $\mathfrak{S}$ is representable, if there exists a manifold $X$ such that $X \cong \mathfrak{X}$ (as groupoid fibrations over $\mathfrak{S}$ ).

Definition 2.6 A morphism of groupoid fibrations $\mathfrak{X} \rightarrow \mathfrak{Y}$ is called a representable submersion, if for every manifold $U$ and every morphism $U \rightarrow \mathfrak{Y}$ the fibered product $V=\mathfrak{X} \times \mathfrak{Y} U$ is representable and the induced morphism of manifolds $V \rightarrow U$ is a submersion.

If the relative dimension $V \rightarrow U$ is always equal to $n \in \mathbb{Z}$, then we call $n$ the relative dimension of $\mathfrak{X} \rightarrow \mathfrak{Y}$. 
Example 2.7 For a Lie group $G$, the canonical morphism $* \rightarrow B G$ is a representable submersion. Here the functor assigns to any smooth manifold $U$ the trivial $G$-bundle over $U$. We can think of $* \rightarrow B G$ as the universal $G$-bundle, because every $G$-bundle $P \rightarrow S$ gives rise to a 2 -fibered product

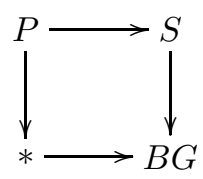

The following lemma will be useful in the future.

Lemma 2.8 (Descent) Let $F$ be a sheaf over $\mathfrak{S}$. Let $X$ be a manifold and $F \rightarrow X$ a morphism. Suppose that $\left\{U_{i} \rightarrow X\right\}$ is a covering family of $X$ and that for every $i$ the sheaf $F_{i}=U_{i} \times_{X} F$ is representable. Then $F$ is representable.

Proof. First note that we can choose a refinement of the covering $\left\{U_{i} \rightarrow X\right\}$ consisting of open subsets of $X$. Replacing the covering by such a refinement, we reduce to the case of a cover $\left\{U_{i} \rightarrow X\right\}$ consisting of open subsets.

Let, as usual, $U_{i j}=U_{i} \times_{X} U_{j}=U_{i} \cap U_{j}$. Define $F_{i j}=U_{i j} \times_{X} F$. Then all $F_{i j}$ are representable. Moreover, all maps $F_{i j} \rightarrow F_{i}$ and $F_{i j} \rightarrow F_{j}$ are (isomorphic to) embeddings of open subsets. Thus we can glue the manifolds $F_{i}$ along the open submanifolds $F_{i j}$ to obtain a manifold representing $F$.

Definition 2.9 A morphism of groupoid fibrations $\mathfrak{X} \rightarrow \mathfrak{Y}$ is an epimorphism if for every $U \rightarrow \mathfrak{Y}$, where $U$ is a manifold, there exists a surjective submersion $V \rightarrow U$ and a 2-commutative diagram

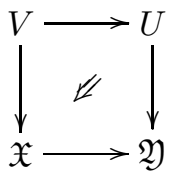

Equivalently, $V$ may be replaced by an open cover of $U$, in this statement.

Remark Let $\mathfrak{X}$ be a category fibered in groupoids over $\mathfrak{S}$. Given a manifold $U \in \mathfrak{S}$ and an object $x \in \mathfrak{X}_{U}$ (we write $x \mid U$ ), the choice of pullbacks of $x$ for all maps $V \rightarrow U$ defines a morphism $U \rightarrow \mathfrak{X}$. Conversely, given a morphism $U \rightarrow \mathfrak{X}$, the image of $\operatorname{id}_{U}$ is an object in the fiber $\mathfrak{X}_{U}$. In this way we identify morphisms $U \rightarrow \mathfrak{X}$ with objects in the fiber $\mathfrak{X}_{U}$.

\section{$2.2 \quad$ Stacks}

Recall the definition of stack [25]:

Definition 2.10 Let $\mathfrak{X} \rightarrow \mathfrak{S}$ be a category fibered in groupoids. We call $\mathfrak{X}$ a stack over $\mathfrak{S}$, if the following three axioms are satisfied:

(i) for any $C^{\infty}$-manifold $X \in \mathfrak{S}$, any two objects $x, y \in \mathfrak{X}$ lying over $X$, and any two isomorphisms $\phi, \psi: x \rightarrow y$ over $X$, such $\phi\left|U_{i}=\psi\right| U_{i}$, for all $U_{i}$ in a covering family $U_{i} \rightarrow X$, we have that $\phi=\psi$;

(ii) for any $C^{\infty}$-manifold $X \in \mathfrak{S}$, any two objects $x, y \in \mathfrak{X}$ lying over $X$, a covering family $U_{i} \rightarrow X$ and, for every $i$, an isomorphism $\phi_{i}: x\left|U_{i} \rightarrow y\right| U_{i}$, such that $\phi_{i}\left|U_{i j}=\phi_{j}\right| U_{i j}$, for all $i, j$, there exists an isomorphism $\phi: x \rightarrow y$, such that $\phi \mid U_{i}=\phi_{i}$, for all $i$; 
(iii) for every $C^{\infty}$-manifold $X$, every covering family $\left\{U_{i}\right\}$ of $X$, every family $\left\{x_{i}\right\}$ of objects $x_{i}$ in the fiber $\mathfrak{X}_{U_{i}}$ and every family of morphisms $\left\{\phi_{i j}\right\}, \phi_{i j}: x_{i}\left|U_{i j} \rightarrow x_{j}\right| U_{i j}$, satisfying the cocycle condition $\phi_{j k} \circ \phi_{i j}=\phi_{i k}$ (which is an equation in the fiber $\mathfrak{X}_{U_{i j k}}$ ), there exists an object $x$ over $X$, together with isomorphisms $\phi_{i}: x \mid U_{i} \rightarrow x_{i}$ such that $\phi_{i j} \circ \phi_{i}=\phi_{j}\left(\right.$ over $U_{i j}$ ).

Note that the isomorphism $\phi$, whose existence is asserted in (ii) is unique, by (i). Similarly, the object $x$, whose existence is asserted in (iii), is unique up to a unique isomorphism, because of (i) and (ii). The object $x$ is said to be obtained by gluing the objects $x_{i}$ according to the gluing data $\phi_{i j}$.

Note also that there are choices to be made for all the pullbacks mentioned in the definition of stacks, but no property depends on any of these choices.

Remark To any covering family $U_{i}$ of $X$, we can associate a groupoid fibration $R$, together with a monomorphism $R \subset X$ of groupoid fibrations, the covering sieve given by $U_{i}$. The stack axioms may be reformulated in terms of covering sieves: thus, a groupoid fibration $\mathfrak{X}$ is a stack if and only if for every covering sieve $R \subset X$, of every object $X \in \mathfrak{S}$, the functor

$$
\operatorname{Hom}_{\mathfrak{S}}(X, \mathfrak{X}) \longrightarrow \operatorname{Hom}_{\mathfrak{S}}(R, \mathfrak{X})
$$

is an equivalence of groupoids. More precisely, $\mathfrak{X}$ satisfies Stack Axiom (i) if and only if (2) is always faithful, $\mathfrak{X}$ satisfies Stack Axiom (ii) if and only if (2) is always full and $\mathfrak{X}$ satisfies Stack Axiom (iii) if and only if (2) is always essentially surjective.

The following lemma is useful in practice.

Lemma 2.11 Let $f: \mathfrak{X} \rightarrow \mathfrak{Y}$ be a morphism of stacks over $\mathfrak{S}$. Suppose given a manifold $U$ and a morphism $U \rightarrow \mathfrak{Y}$ which is an epimorphism. If the fibered product $V=\mathfrak{X} \times_{\mathfrak{Y}} U$ is representable and $V \rightarrow U$ is a submersion, then $f$ is a representable submersion.

If $V \rightarrow U$ has relative dimension $n$, then so does $f$.

Proof. Let $W \rightarrow \mathfrak{Y}$ be an arbitrary morphism, where $W$ is a manifold. First we have to show that the fibered product $F=\mathfrak{X} \times \mathfrak{Y} W$ is representable. By the fact that $U \rightarrow \mathfrak{Y}$ is an epimorphism, we can choose a covering family $\left\{W_{i} \rightarrow W\right\}$ of $W$ and morphisms $\phi_{i}: W_{i} \rightarrow U$ making the diagram

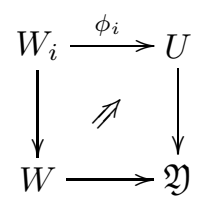

commute (which involves, of course, also a choice of a 2-arrow, for every $i$ ). By Lemma 2.8, it suffices to prove that $F_{i}=W_{i} \times_{W} F$ is representable, for all $i$. But $F_{i}=W_{i} \times_{U} V$, as can be seen from the cartesian cube

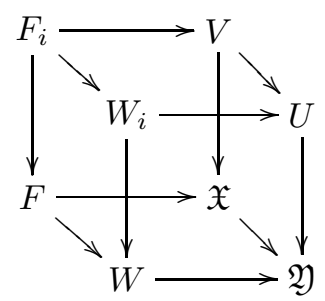

and so, indeed, $F_{i}$, and hence $F$ is representable. 
Now the fact that $F \rightarrow W$ is a submersion, follows from the fact that for every $i$ the map $F_{i} \rightarrow W_{i}$ is a submersion, because being a submersion is a local property. But $F_{i} \rightarrow W_{i}$ is a submersion as a pull back of the submersion $V \rightarrow U$.

Example 2.12 Let $G$ be a Lie group and $H$ a closed Lie subgroup. The induced morphism $B H \rightarrow B G$ is a representable submersion. To see this, let us apply Lemma 2.11. Note that $* \rightarrow B G$ is an epimorphism, because every $G$-bundle is locally trivial. Note also that we have a cartesian diagram

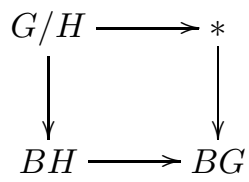

because the reductions of structure group from $G$ to $H$ of the trivial $G$-bundle over a manifold $U$ are classified by the maps $U \rightarrow G / H$. Since $G / H$ is a manifold, $G / H \rightarrow *$ is a submersion, which finishes the proof. The relative dimension of $B H \rightarrow B G$ is equal to $\operatorname{dim} G-\operatorname{dim} H$.

Two stacks $\mathfrak{X}$ and $\mathfrak{Y}$ over $\mathfrak{S}$ are said to be isomorphic if they are equivalent as categories over $\mathfrak{S}$. This means that there exist morphisms $f: \mathfrak{X} \rightarrow \mathfrak{Y}$ and $g: \mathfrak{Y} \rightarrow \mathfrak{X}$ and 2-isomorphisms $\theta: f \circ g \Rightarrow \operatorname{id}_{\mathfrak{Y}}$ and $\eta: g \circ f \Rightarrow \operatorname{id}_{\mathfrak{X}}$.

Proposition 2.13 For stacks $\mathfrak{X}$ and $\mathfrak{Y}$ over $\mathfrak{S}$ to be isomorphic, it suffices that there exists a morphism $f: \mathfrak{X} \rightarrow \mathfrak{Y}$ satisfying the two conditions:

(i) for any two objects $x, x^{\prime}$ of $\mathfrak{X}$, lying over the same object $U$ of $\mathfrak{S}$, and any arrow $\eta: f(x) \rightarrow$ $f\left(x^{\prime}\right)$ in $\mathfrak{Y}_{U}$, there exists a unique arrow $x \rightarrow x^{\prime}$ mapping to $\eta$ under $f$ (we say $f$ is fully faithful or a monomorphism);

(ii) for every object $y$ of $\mathfrak{Y}$, lying over $S \in \mathfrak{S}$, there exists a covering family $\left\{U_{i}\right\}$ of $S$ and objects $x_{i}$ of $\mathfrak{X}$ lying over $U_{i}$, such that $f\left(x_{i}\right) \cong y \mid U_{i}$, for all $i$ (we say that $f$ is an epimorphism).

A morphism satisfying both these conditions is called an isomorphism of stacks.

\section{$2.3 \quad$ Differentiable stacks}

Let $\mathfrak{X}$ be a groupoid fibration over $\mathfrak{S}$. Recall that we may think of $x / S$ equivalently as a morphism of groupoid fibrations $x: S \rightarrow \mathfrak{X}$.

For $x / S$ and $y / T$, consider the fibered product

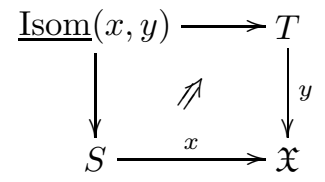

For an $\mathfrak{X}$-family $x$, parametrized by $S$, we call $\underline{\operatorname{Isom}}(x, x) \rightrightarrows S$ the symmetry groupoid of $x$. A priori, $\underline{\operatorname{Isom}}(x, x)$ is just a groupoid fibration over $\mathfrak{S}$, but it may be hoped that it is (represented by) a Lie groupoid. Note that we have a cartesian diagram

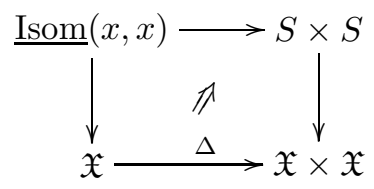


Thus, ultimately, properties of the diagonal $\Delta: \mathfrak{X} \rightarrow \mathfrak{X} \times \mathfrak{X}$, will assure that the symmetry groupoids $\underline{\operatorname{Isom}}(x, x)$ are manifolds, at least if $S \rightarrow \mathfrak{X}$ is sufficiently well-behaved.

Lemma 2.14 Let $f: \mathfrak{X} \rightarrow \mathfrak{Y}$ be a representable submersion of stacks over $\mathfrak{S}$. Then the following are equivalent:

(i) $f$ is an epimorphism;

(ii) for every manifold $U \rightarrow \mathfrak{Y}$ the submersion $V \rightarrow U$, where $V$ is the fibered product $V=$ $\mathfrak{X} \times \mathfrak{Y} U$, is surjective;

(iii) for some manifold $U \rightarrow \mathfrak{Y}$, where $U \rightarrow \mathfrak{Y}$ is an epimorphism, the submersion $V \rightarrow U$ is surjective.

A representable submersion satisfying these conditions is called a surjective representable submersion.

Proof. This follows from the fact that a submersion between manifolds is an epimorphism of stacks if and only if it is surjective. We also use that to be an epimorphism is a local property.

Definition 2.15 A stack $\mathfrak{X}$ over $\mathfrak{S}$ is called differentiable or a $C^{\infty}$-stack, if there exists a manifold $X$ and a surjective representable submersion $x: X \rightarrow \mathfrak{X}$. Such a manifold $X$, together with the structure morphism $X \rightarrow \mathfrak{X}$ is called a presentation of $\mathfrak{X}$ or an atlas for $\mathfrak{X}$, and such a family $x / X$ is called a versal family.

Alternatively, one can describe a differentiable stack in a slightly weaker condition.

Proposition 2.16 A stack over $\mathfrak{S}$ is a differentiable stack, if there exists an $\mathfrak{X}$-family $x / X$, such that

(i) the symmetry groupoid $\underline{\operatorname{Isom}}(x, x)$ is representable, and the projections $\underline{\operatorname{Isom}}(x, x) \rightarrow X$ are submersions;

(ii) the morphism $x: X \rightarrow \mathfrak{X}$ is an epimorphism. I.e., for every $\mathfrak{X}$-family $y / S$, there exists a covering family $U_{i}$ of $S$, and morphisms $\phi_{i}: U_{i} \rightarrow X$, such that $y \mid U_{i} \cong \phi_{i}^{*} x$.

Proof. Given such an $\mathfrak{X}$-family $x / X$, it suffices to show that $x: X \rightarrow \mathfrak{X}$ is representable submersion. This follows from Lemma 2.11 since $x: X \rightarrow \mathfrak{X}$ is epimorphism, $X \times_{\mathfrak{X}} X$ is representable and $X \times \mathfrak{X} X \rightarrow X$ is a submersion.

The converse is obvious.

The 2-category of differentiable stacks is the full sub-2-category of the 2-category of groupoid fibrations over $\mathfrak{S}$ consisting of differentiable stacks.

Given a differentiable stack $\mathfrak{X}$, a versal family $x / X$ gives rise to a Lie groupoid $\underline{\operatorname{Isom}}(x, x) \rightrightarrows X$ in a canonical way. The points of $\underline{\operatorname{Isom}}(x, x)$ are by definition triples $\left(y, \phi, y^{\prime}\right)$, where $y$ and $y^{\prime}$ are points of $X$ and $\phi: x|y \rightarrow x| y^{\prime}$ is a morphism in the groupoid $\mathfrak{X}_{*}$ (the fiber of $\mathfrak{X}$ over $* \in \mathfrak{S}$ ). So it is clear how to define the composition:

$$
\left(y, \phi, y^{\prime}\right) \circ\left(y^{\prime}, \psi, y^{\prime \prime}\right)=\left(y, \psi \circ \phi, y^{\prime \prime}\right) .
$$

To see that this, indeed, defines the structure of a Lie groupoid on $\underline{\operatorname{Isom}}(x, x) \rightrightarrows X$, the quickest way is to note that for every manifold $U$, evaluating at $U$ we get a (set-theoretic) groupoid $\underline{\text { Isom }}(x, x)(U) \rightrightarrows X(U)$, defined by the same formula (3) and compatible with all maps $V \rightarrow U$.

A morphism $\mathfrak{X} \rightarrow \mathfrak{Y}$ of differentiable stack is representable if one of the following equivalent conditions is satisfied:

1. there is a presentation $Y \rightarrow \mathfrak{Y}$ such that $\mathfrak{X} \times \mathfrak{Y} Y$ is representable; 
2. for any representable submersion $Y \rightarrow \mathfrak{Y}, \mathfrak{X} \times_{\mathfrak{Y}} Y$ is representable.

For instance, the diagonal map $\mathfrak{X} \rightarrow \mathfrak{X} \times \mathfrak{X}$ of a differentiable stack $\mathfrak{X}$ is always representable.

A representable morphism $\mathfrak{X} \rightarrow \mathfrak{Y}$ is called proper if there exists a presentation $Y \rightarrow \mathfrak{Y}$ such that the base change $X \rightarrow Y$ is proper. If this is the case, $X \rightarrow Y$ is proper for all representable submersion $Y \rightarrow \mathfrak{Y}$.

Example 2.17 Let $\mathfrak{X}_{g}$ be the following groupoid fibration: objects are fiber bundles $X \rightarrow S$ with fibers being isomorphic to a fixed connected surface $Y$ of genus $g$. Morphisms are commutative diagrams

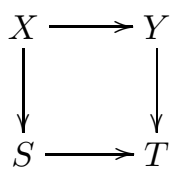

such that $X \rightarrow Y \times_{T} S$ is an isomorphism. Consider the constant family $Y \rightarrow *$. Then $* \stackrel{Y}{\rightarrow} \mathfrak{X}_{g}$ is an epimorphism, because every family of surfaces is locally trivial. But $* \stackrel{Y}{\rightarrow} \mathfrak{X}_{g}$ is not a representable submersion since the symmetry groupoid of this family is the diffeomorphism group of $Y$, which is not a finite dimensional manifold. So $\mathfrak{X}_{g}$ is not a differentiable stack.

\subsection{Torsors for Lie groupoids}

Next, we show how to get a differentiable stack starting from a Lie groupoid. (This is, in fact, a generalization of passing from $G$ to $B G$.)

Definition 2.18 Let $\Gamma \rightrightarrows M$ be a Lie groupoid and $S$ a manifold. A $\Gamma$-torsor over $S$ is a manifold $P$, together with a surjective submersion $\pi: P \rightarrow S$ and a (right) action of $\Gamma$ on $P$, such that for all $p, p^{\prime} \in P$, such that $\pi(p)=\pi\left(p^{\prime}\right)$, there exists a unique $\gamma \in \Gamma$, such that $p \cdot \gamma$ is defined and $p \cdot \gamma=p^{\prime}$.

We call the map $P \rightarrow M$ of the $\Gamma$-torsor $P$ the anchor map and denote it by $a: P \rightarrow M$. (In the theory of symplectic groupoids the anchor map is also called the "momentum map" [30].) And the surjective submersion $\pi: P \rightarrow S$ is called the structure map.

Remark Think of a $\Gamma$-torsor as follows. View an element $p \in P$ as an arrow eminating at $\pi(p)$ and terminating at $a(p)$. Then view the action of $\Gamma$ on $P$ as composing arrows.

Definition 2.19 Let $\pi: P \rightarrow S$ and $\rho: Q \rightarrow T$ be $\Gamma$-torsors. A morphism of $\Gamma$-torsors from $Q$ to $P$ is given by a commutative diagram of differentiable maps

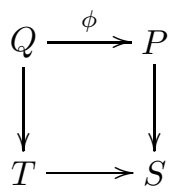

such that $\phi$ is $\Gamma$-equivariant.

Note that for a morphism of $\Gamma$-torsors the diagram (4) is necessarily a pullback diagram.

The $\Gamma$-torsors form a category with respect to this notion of morphism. In particular, we now know what it means for two $\Gamma$-torsors to be isomorphic. 
Example 2.20 (trivial torsors) Let $f: S \rightarrow M$ be a smooth map. Given $f$, we can induce over $S$ in a canonical way a $\Gamma$-torsor, which we call the trivial $\Gamma$-torsor given by $f$.

Simply define $P$ to be the fibered product $P=S \times_{f, M, s} \Gamma$. The structure map $\pi: P \rightarrow S$ is the first projection. The anchor map of the $\Gamma$-action is the second projection followed by the target map $t$. The action is then defined by

$$
(s, \gamma) \cdot \delta=(s, \gamma \cdot \delta) .
$$

One checks that this is, indeed, a $\Gamma$-torsor over $S$.

Of course, we can take $S=M$ and $f$ the identity map of $M$. Then we get the universal trivial $\Gamma$-torsor, whose base is $M$. The structure morphism and the anchor map of the universal $\Gamma$-torsor are, respectively, $t, s: \Gamma \rightarrow M$.

Let $\pi: P \rightarrow S$ be an arbitrary $\Gamma$-torsor over the manifold $S$. One checks that every section $s: S \rightarrow P$ of $\pi$ can be used to construct an isomorphism between the $\Gamma$-torsor $P$ and the trivial $\Gamma$-torsor over $S$ given by $a \circ s$, where $a: P \rightarrow M$ is the anchor map of $P$.

Since every surjective submersion admits local sections, we see that every $\Gamma$-torsor is locally trivial.

Let us denote the category of $\Gamma$-torsors by $B \Gamma$. There is a canonical functor $B \Gamma \rightarrow \mathfrak{S}$ given by mapping a torsor $P \rightarrow S$ to the underlying manifold $S$.

The following proposition provides us with plenty of examples of differentiable stacks. Theorem 2.22 below indeed shows that it provides us with all examples of differentiable stacks.

Proposition 2.21 For every Lie groupoid $\Gamma \rightrightarrows M$, the category of $\Gamma$-torsors $B \Gamma$ is a differentiable stack.

Proof. The fact that $B \Gamma$ is fibered in groupoids over $\mathfrak{S}$ follows from the fact that diagrams such as (4) are always cartesian. Note that given a $\Gamma$-torsor $P \rightarrow S$ and a morphism of manifolds $T \rightarrow S, T \times{ }_{S} P \rightarrow T$ is naturally a $\Gamma$-torsor over $T$.

To check the stack axioms, one has to prove that one can glue together $\Gamma$-torsors and morphisms of $\Gamma$-torsors. This is rather standard and will be omitted.

Finally we need to prove that $B \Gamma$ admits a presentation. For this, we take the universal trivial torsor. We shall construct a morphism $M \rightarrow B \Gamma$. This means defining for every manifold $S$ a map $M(S) \rightarrow B \Gamma(S)$. This we do by assigning to any smooth map $a: S \rightarrow M$ (i.e. object of $M(S)$ ) the trivial $\Gamma$-torsor over $S$, which is an object of $B \Gamma(S)$. Alternatively, we can use the universal trivial $\Gamma$-torsor, which gives rise to the morphism $M \rightarrow B \Gamma$ directly, via the correspondence between objects of the fiber $B \Gamma(U)$ and morphisms $U \rightarrow B \Gamma(U)$ (see the remark following Definition 2.9).

Now that we have a morphism $M \rightarrow B \Gamma$ from a manifold $M$, it remains to prove that this morphism is a surjective representable submersion. To prove that $M \rightarrow B \Gamma$ is an epimorphism, means proving that every $\Gamma$-torsor is locally trivial. This we have done already. By Proposition 2.16 , it now suffices to prove that the fibered product

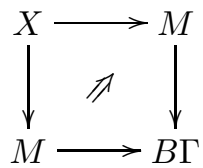

is representable and that the maps $X \rightrightarrows M$ are submersions.

Let $S$ be an arbitrary manifold. Then $X(S)$ is the set of triples $(a, \gamma, b)$, where $a, b: S \rightarrow M$ are $C^{\infty}$ maps and $\gamma: Q_{a} \rightarrow Q_{b}$ is a morphism of $\Gamma$-torsors over $S$, where $Q_{a}$ and $Q_{b}$ are the trivial $\Gamma$-torsors over $S$ given by $a$ and $b$, respectively. One checks that this set is canonically identified with $\Gamma(S)$, the set of $C^{\infty}$-maps from $S$ to $\Gamma$. 
Thus we have that $X \cong \Gamma$ as stacks over $\mathfrak{S}$, and so $X$ is representable. To check that the two projections $X \rightarrow M$ are submersions, note that they are identified with $s, t: \Gamma \rightarrow M$, under this isomorphism. Since $s$ and $t$ are submersions, we are done.

Remark (1) Note that in the course of the proof we have seen that we have a cartesian diagram

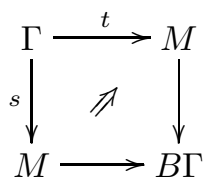

Thus $\Gamma \rightrightarrows M$ is (isomorphic to) the Lie groupoid arising from the atlas $M \rightarrow B \Gamma$.

(2) From the above proof, we see that for a given $a: S \rightarrow M$ the corresponding trivial torsor over $S$ corresponds to the composition of morphisms

$$
S \stackrel{a}{\longrightarrow} M \stackrel{\pi}{\longrightarrow} B \Gamma .
$$

Theorem 2.22 Let $\mathfrak{X}$ be a differentiable stack and $x / X$ a versal family for $\mathfrak{X}$. Then

$$
\mathfrak{X} \cong B \underline{\operatorname{Isom}}(x, x),
$$

as groupoid fibrations over $\mathfrak{S}$.

PROOF. We shall prove that the functor $f$ :

$$
\begin{aligned}
\mathfrak{X} \longrightarrow B \underline{\operatorname{Isom}}(x, x) & \\
y & \longmapsto \underline{\operatorname{Isom}}(x, y),
\end{aligned}
$$

provides us with the required isomorphism of groupoid fibrations.

Since $x: X \rightarrow \mathfrak{X}$ is a representable submersion, it follows that $\underline{\operatorname{Isom}}(x, y)$ is representable. The fact that $\underline{\operatorname{Isom}}(x, x)$ acts simply transitively on $\underline{\operatorname{Isom}}(x, y)$ is clear. Thus, $\underline{\operatorname{Isom}}(x, y)$ is, in fact, an $\underline{\operatorname{Isom}}(x, x)$-torsor.

It remains to prove that (5) is an equivalence of categories. Since both groupoid fibrations are stacks, we can use the local criterion: Proposition 2.13, i.e. to prove that $f$ is a monomorphism and an epimorphism.

For the monomorphism property, let $y, y^{\prime}: S \rightarrow \mathfrak{X}$ be two objects of $\mathfrak{X}$ lying over $S$. Let $Q$ and $Q^{\prime}$ be the Isom $(x, x)$-torsors induced by $y$ and $y^{\prime}$ over $S$. We need to show that any isomorphism of torsors $\phi: Q \rightarrow Q^{\prime}$ comes from a 2-isomorphism $\theta: y \rightarrow y^{\prime}$. This follows from the fact that Isom $\left(y, y^{\prime}\right)$ is a sheaf: choose a covering $\left\{U_{i}\right\}$ of $S$ trivializing the torsor $Q$. Then $\phi$ gives rise to isomorphisms $\theta_{i}: y\left|U_{i} \rightarrow y^{\prime}\right| U_{i}$. One checks that the $\theta_{i}$ glue together, giving rise to $\theta$.

For the epimorphism property, suppose $Q \rightarrow S$ is an $\underline{\operatorname{Isom}}(x, x)$-torsor over $S$. Then there exists a cover $\left\{U_{i}\right\}$ of $S$ and sections $s_{i}: U_{i} \rightarrow Q$, trivializing $Q$ over $\left\{U_{i}\right\}$. The sections $s_{i}$ induce morphisms $x_{i}: U_{i} \rightarrow \mathfrak{X}$ (which are the compositions $U_{i} \rightarrow X \stackrel{x}{\rightarrow} \mathfrak{X}$ ) identifying $Q \mid U_{i}$ with $x_{i}^{*} X$. Thus we see that every $\underline{\operatorname{Isom}}(x, x)$-torsor over $S$ comes locally from objects of $\mathfrak{X}$, proving that $f$ is an epimorphism.

Definition 2.23 For a differentiable stack $\mathfrak{X}$, if the diagonal $\mathfrak{X} \rightarrow \mathfrak{X} \times \mathfrak{X}$ is proper, we call $\mathfrak{X}$ separated or Hausdorff.

For a differentiable stack $\mathfrak{X}$, the diagonal $\mathfrak{X} \rightarrow \mathfrak{X} \times \mathfrak{X}$ is always representable. Indeed if $X_{1} \rightrightarrows X_{0}$ is a Lie groupoid representing $\mathfrak{X}$, then $\mathfrak{X}_{\mathfrak{X} \times \mathfrak{X}}\left(X_{0} \times X_{0}\right) \cong X_{1}$ and the base change map is $s \times t: X_{1} \rightarrow X_{0} \times X_{0}$. Hence $\mathfrak{X}$ is separated if and only if $X_{1} \rightrightarrows X_{0}$ is a proper groupoid.

In the definition of Metzler [34], all differentiable stacks are required to be separated. We believe that this is too restrictive. Many interesting differentiable stacks are not separated. 


\subsection{Morita equivalence}

We have now established procedures to go back and forth between Lie groupoids and differentiable stacks. Given a differentiable stack $\mathfrak{X}$, we choose a presentation $X_{0} \rightarrow \mathfrak{X}$ and form the associated Lie groupoid $X_{1} \rightrightarrows X_{0}$ by taking the fibered product. Conversely, starting with a Lie groupoid $\Gamma \rightrightarrows M$, we construct the differentiable stack $B \Gamma$ of $\Gamma$-torsors, which comes with a canonical presentation, giving back the groupoid $\Gamma \rightrightarrows M$ we started with (up to isomorphism). It remains to see when exactly two different Lie groupoids give rise to isomorphic differentiable stacks, or put another way, what relationship there is between various Lie groupoids arising from various presentations of a differentiable stack.

Definition 2.24 Let $X_{\bullet}$ and $Y_{\bullet}$ be Lie groupoids. A morphism $\phi_{\bullet}: X_{\bullet} \rightarrow Y_{\bullet}$ is called a Morita morphism, if

(i) $\phi_{0}: X_{0} \rightarrow Y_{0}$ is a surjective submersion;

(ii) the diagram

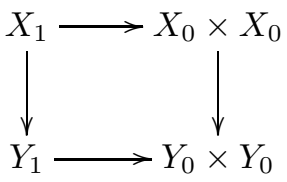

is cartesian.

Definition 2.25 Two Lie groupoids $X_{\bullet}$ and $Y_{\bullet}$ are called Morita equivalent, if there exists a third Lie groupoid $Z_{\bullet}$ and Morita morphisms $Z_{\bullet} \rightarrow X_{\bullet}$ and $Z_{\bullet} \rightarrow Y_{\bullet}$

Theorem 2.26 Let $X_{\bullet}$ and $Y_{\bullet}$ be Lie groupoids. Let $\mathfrak{X}$ and $\mathfrak{Y}$ be the associated differentiable stacks, i.e., $\mathfrak{X}$ is the stack of $X_{\bullet}$-torsors and $\mathfrak{Y}$ the stack of $Y_{\bullet}$-torsors. Then the following are equivalent:

(i) the differentiable stacks $\mathfrak{X}$ and $\mathfrak{Y}$ are isomorphic;

(ii) the Lie groupoids $X_{\bullet}$ and $Y_{\bullet}$ are Morita equivalent;

(iii) there exists a manifold $Q$ together with two $C^{\infty}$ maps $f: Q \rightarrow X_{0}$ and $g: Q \rightarrow Y_{0}$ and (commuting) actions of $X_{1}$ and $Y_{1}$ (the action of $X_{1}$ comprising $f$ and the action of $Y_{1}$ comprising $g$ ), in such a way that $Q$ is at the same time an $X_{\bullet}$-torsor over $Y_{0}$ (via g) and a $Y_{\bullet}$-torsor over $X_{0}$ (via $\left.f\right)$. We call such $a Q$ an $X_{\bullet}-Y_{\bullet}$-bitorsor.

Proof. Let us start by proving that (i) implies (iii). Choose an isomorphism identifying $\mathfrak{X}$ with $\mathfrak{Y}$. Then let $Q$ be the fibered product $Q=Y_{0} \times_{\mathfrak{X}} X_{0}$. One checks that $Q$ is a bitorsor.

To prove that (iii) implies (ii), choose a bitorsor $Q$. Let $Q_{1}$ be the fibered product $Q_{1}=$ $Y_{1} \times_{Y_{0}} Q \times_{X_{0}} X_{1}$. There is a canonical way to define a Lie groupoid $Q_{1} \rightrightarrows Q$, together with Morita equivalences $Q_{\bullet} \rightarrow Y_{\bullet}$ and $Q_{\bullet} \rightarrow X_{\bullet}$.

One also proves that (ii) implies (iii). This follows from the following two facts: (1) if $\phi$ : $X_{\bullet} \rightarrow Y_{\bullet}$ is a Morita morphism, then $Q=X_{0} \times_{Y_{0}, s} Y_{1}$ is naturally an $X_{\bullet}-Y_{\bullet}$-bitorsor; (2) if $Q$ is a $X_{\bullet}-Y_{\bullet}$-bitorsor, and $Q^{\prime}$ is an $Y_{\bullet}-Z_{\bullet}$-bitorsor, then $\left(Q \times_{Y_{0}} Q^{\prime}\right) / Y_{1}$ is an $X_{\bullet}$ - $Z$ - -bitorsor.

Finally, we need to prove that (iii) implies (i). Given an $X_{\bullet}$-torsor $F$ over $U$, let $E=X_{1} \backslash\left(Q \times_{X_{0}}\right.$ $F)$. Then $E$ is a $Y_{\bullet}$-torsor over $U$, where the anchor map $E \rightarrow Y_{0}$ is $a([q, f])=g(q)$ and the $Y_{1^{-}}$ action is $y \cdot[q, f]=\left[q \cdot y^{-1}, f\right]$. Also it is clear that a morphism of $X_{\bullet}$-torsors $F_{1} \rightarrow F_{2}$ induces a morphism of $Y_{\bullet}$-torsor $E_{1} \rightarrow E_{2}$ in a canonical way. Thus one obtains a functor, which can be easily seen to be an equivalence of categories.

Remark Note that (iii) is the definition of Morita equivalence used in a lot of literature on operator algebras $[22,31]$. 


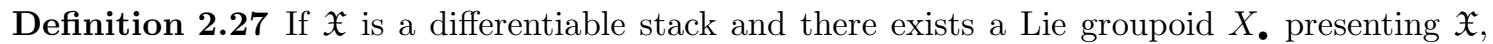
such that $X_{0}$ and $X_{1}$ both have constant dimensions, then we call $\operatorname{dim} \mathfrak{X}=2 \operatorname{dim} X_{0}-\operatorname{dim} X_{1}$ the dimension of $\mathfrak{X}$.

We see that, from Theorem 2.26, $\operatorname{dim} \mathfrak{X}$ is independent of the presentation of $\mathfrak{X}$, and therefore is well-defined.

Remark Note that $\operatorname{dim} \mathfrak{X}$ can also be written as the base dimension minus the fibre dimension of the representing groupoid $X_{1} \rightrightarrows X_{0}$, which is also the orbit space dimension minus the isotropy group dimension. Also $\operatorname{dim} \mathfrak{X}$ can be negative. In particular, if $G$ is a Lie group of dimension $n$, the stack $B G$ is of dimension $-n$.

\subsection{Dictionary}

Theorem 2.26 is only the beginning of a dictionary between differentiable stacks and Lie groupoids. We will now list a few propositions that give more precise information, in particular with respect to morphisms and 2-isomorphisms.

All these results are standard in stack theory. Proofs are elementary, but usually tedious, and we omit them.

\section{The 2-category of Lie groupoids}

Recall the notion of natural equivalence between groupoid morphisms:

Definition 2.28 Let $\phi: X_{\bullet} \rightarrow Y_{\bullet}$ and $\psi: X_{\bullet} \rightarrow Y_{\bullet}$ be two morphisms of Lie groupoids. A natural equivalence from $\phi$ to $\psi$, notation $\theta: \phi \Rightarrow \psi$, is a $C^{\infty}$ map $\theta: X_{0} \rightarrow Y_{1}$ such that for every $x \in X_{1}$ we have

$$
\theta(s(x)) * \psi(x)=\phi(x) * \theta(t(x)) .
$$

Fixing the Lie groupoids $X_{\bullet}$ and $Y_{\bullet}$, the morphisms and natural equivalences form a category $\operatorname{Hom}\left(X_{\bullet}, Y_{\bullet}\right)$, which is a (set-theoretic) groupoid. With this notion of morphism groupoid, the Lie groupoids form a 2-category.

\section{The Dictionary Lemmas}

We consider two Lie groupoids $X$. and $Y$. with associated differentiable stacks $\mathfrak{X}$ and $\mathfrak{Y}$, respectively. The dictionary lemmas relate groupoid morphisms $X_{\bullet} \rightarrow Y_{\bullet}$ to stack morphisms $\mathfrak{X} \rightarrow \mathfrak{Y}$.

The first Dictionary Lemma says that a morphism of Lie groupoids induces a morphism of associated differentiable stacks, unique up to unique 2-isomorphism:

Lemma 2.29 (First Dictionary Lemma) Let $\phi: X_{\bullet} \rightarrow Y_{\bullet}$ be a morphism of Lie groupoids. Let $\mathfrak{X}$ and $\mathfrak{Y}$ be differentiable stacks associated to $X_{\bullet}$ and $Y_{\bullet}$, respectively. Then there exists a morphism of stacks $f: \mathfrak{X} \rightarrow \mathfrak{Y}$ and a 2-isomorphism

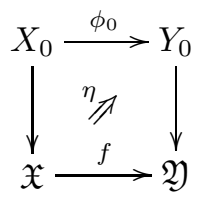


such that the cube

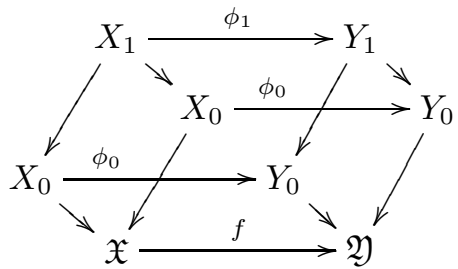

2-commutes. If $\left(f^{\prime}, \eta^{\prime}\right)$ is another pair satisfying these properties, then there is a unique 2isomorphism $\theta: f \Rightarrow f^{\prime}$ such that $\theta * \eta^{\prime}=\eta$.

The second and third Dictionary Lemmas treat the converse:

Lemma 2.30 (Second Dictionary Lemma) Let $f: \mathfrak{X} \rightarrow \mathfrak{Y}$ be a morphism of stacks, $\phi_{0}$ : $X_{0} \rightarrow Y_{0}$ a morphism of manifolds and $\eta$ a 2-isomorphism as in (6). Then there exists a unique morphism of Lie groupoids $\phi_{1}: X_{1} \rightarrow Y_{1}$ covering $\phi_{0}$ and making the cube (7) 2-commutative.

Lemma 2.31 (Third Dictionary Lemma) Let $f: \mathfrak{X} \rightarrow \mathfrak{Y}$ be a morphism of stacks. Let $\phi$ : $X_{\bullet} \rightarrow Y_{\bullet}$ and $\psi: X_{\bullet} \rightarrow Y_{\bullet}$ be two morphisms of Lie groupoids. Let $\eta$ and $\eta^{\prime}$ be 2-isomorphisms, where $(\phi, \eta)$ and $\left(\psi, \eta^{\prime}\right)$ both form 2-commutative cubes such as (6). Then there exists a unique natural equivalence $\theta: \phi \Rightarrow \psi$ such that the diagram

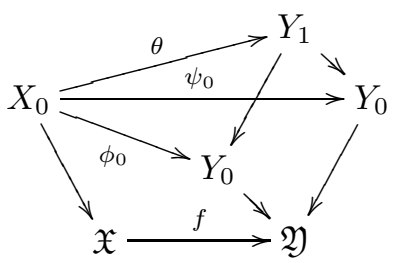

2-commutes.

\subsection{Differentiable Spaces}

Differentiable spaces are generalizations of manifolds. The are differentiable stacks whose isotropy groups are trivial. They occur when one tries to define the quotient of an equivalence relation which is "of Lie type" (i.e. is given by a Lie groupoid) but the usual quotient has bad properties (i.e. is not a manifold or not a principal bundle quotient). Differentiable spaces have slightly better properties than manifolds. The main advantage is that Lemma 2.35 holds for them.

Definition 2.32 A sheaf over $\mathfrak{S}$, which, considered as a stack over $\mathfrak{S}$ is differentiable, is called a differentiable space.

Thus a sheaf $F$ is a differentiable space if there exists a manifold $X$ and a surjective representable submersion $X \rightarrow F$.

Example 2.33 If a Lie group acts on a manifold freely, but not properly, we get a differentiable space.

Proposition 2.34 The differentiable stack $\mathfrak{X}$ defined by a Lie groupoid $X$. is (isomorphic to) a differentiable space if and only if $X_{\bullet}$ is a Lie equivalence relation (i.e. $X_{1} \rightarrow X_{0} \times X_{0}$ is injective).

In particular, if $X_{\bullet}$ and $Y_{\bullet}$ are Morita equivalent Lie groupoids, then $X_{\bullet}$ is an equivalence relation if and only if $Y$. is. 
Thus we may think of differentiable spaces as Lie equivalence relations up to Morita equivalence.

Lemma 2.35 (Submersive descent for differentiable spaces) Let $F$ be a sheaf over $\mathfrak{S}$. Let $X$ be a manifold and $F \rightarrow X$ a morphism. Suppose that $U \rightarrow X$ is a surjective submersion of manifolds and that the sheaf $G=U \times_{X} F$ is a differentiable space. Then $F$ is a differentiable space.

Note that there is no corresponding statement for manifolds. For manifolds we only have étale descent (Lemma 2.8).

Definition 2.36 Let $\mathfrak{X}$ and $\mathfrak{Y}$ be stacks over $\mathfrak{S}$. We call a morphism $f: \mathfrak{X} \rightarrow \mathfrak{Y}$ weakly representable, if for every representable submersion $U \rightarrow \mathfrak{Y}$, where $U$ is a manifold, the fibered product $V=\mathfrak{X} \times_{\mathfrak{Y}} U$ is isomorphic to a differentiable space.

Proposition 2.37 Let $\mathfrak{X}$ and $\mathfrak{Y}$ be differentiable stacks. The morphism $f: \mathfrak{X} \rightarrow \mathfrak{Y}$ is weakly representable if there exists a presentation $Y \rightarrow \mathfrak{Y}$ such that $X=\mathfrak{X} \times_{\mathfrak{Y}} Y$ is isomorphic to a differentiable space.

Proof. The proof is very similar to the proof of Lemma 2.11. We need to use submersive descent for differentiable spaces.

Example 2.38 Representable morphisms are weakly representable. In particular, the diagonal $\mathfrak{X} \rightarrow \mathfrak{X} \times \mathfrak{X}$ of a differentiable stack is weakly representable, and any $C^{\infty}$-map of manifolds is weakly representable.

Moreover, any morphism from a differentiable space to a differentiable stack is weakly representable, and any morphism of differentiable stacks which is faithful is weakly representable.

Remark We get a weaker notion of differentiable stack if we work with groupoids where $X_{0}$ and $X_{1}$ are differentiable spaces rather than manifolds. Equivalently, we can relax the condition that the diagonal $\mathfrak{X} \rightarrow \mathfrak{X} \times \mathfrak{X}$ be representable to it being weakly representable. We could call these stacks weakly differentiable stacks.

For example, the quotient $\mathbb{R} / \mathbb{Q}$ is a differentiable space but not a manifold. It is also a group. The associated stack $B(\mathbb{R} / \mathbb{Q})$ is weakly differentiable but not differentiable.

Remark It would be interesting to investigate the relationship between differentiable spaces and Souriau's diffeology structures [47].

\section{Homology and cohomology}

Here our goal is to define the cohomology of a differentiable stack with values in a sheaf (or a complex of sheaves) of abelian groups. Of particular interest is the de Rham complex, which gives rise to de Rham cohomology.

Then we pass to Lie groupoids and define the cohomology of a Lie groupoid with values in a sheaf of abelian groups. This cohomology is Morita invariant. For any complex of sheaves of abelian groups, we also define a double complex and its associated cohomology groups. These cohomology groups are not necessarily Morita invariant, but they will be if all component sheaves of the complex are acyclic on manifolds. An example of this is the de Rham complex. Thus de Rham cohomology of a groupoid is also Morita invariant. 


\subsection{Sheaves over stacks and their cohomology}

Let $\mathfrak{X}$ be a differentiable stack. We endow the category $\mathfrak{X}$ with a Grothendieck topology defined as follows: call a family $\left\{x_{i} \rightarrow x\right\}$ of morphisms in $\mathfrak{X}$ a covering family of the object $x \in \mathfrak{X}$, if the image family $\left\{U_{i} \rightarrow U\right\}$ in $\mathfrak{S}$ is a covering family, i.e. is a family of étale maps such that $\amalg U_{i} \rightarrow U$ is surjective. One checks that, indeed, the axioms of a topology are satisfied. Thus we may now speak of sheaves over $\mathfrak{X}$ : i.e. contravariant functors $\mathfrak{X} \rightarrow$ (sets) satisfying the sheaf axioms. We get the category (sheaves $/ \mathfrak{X}$ ) of sheaves over $\mathfrak{X}$.

Remark 3.1 Let $F$ be a sheaf over the stack $\mathfrak{X}$. Consider $F$ as a category fibered in groupoids $F \rightarrow \mathfrak{X}$. Then, by composing with $\mathfrak{X} \rightarrow \mathfrak{S}$, we may turn $F$ into a category fibered in groupoids over $\mathfrak{S}$. One checks that $F$ is then a stack over $\mathfrak{S}$ and that $F \rightarrow \mathfrak{X}$ is a morphism of stacks. Moreover, $F \rightarrow \mathfrak{X}$ is faithful.

Conversely, if $f: \mathfrak{Y} \rightarrow \mathfrak{X}$ is a faithful morphism of stacks over $\mathfrak{S}$, we may associate a sheaf $F$ over $\mathfrak{X}$ defined by $F(x)=\{(y, \phi) \mid \phi: x \rightarrow f(y)\} / \sim$, where $(y, \phi) \sim\left(y^{\prime}, \phi^{\prime}\right)$ if there exists $\eta: y \rightarrow y^{\prime}$ such that $f(\eta) \circ \phi=\phi^{\prime}$. We call $F$ the sheaf of sections of $f: \mathfrak{Y} \rightarrow \mathfrak{X}$.

Thus we get an equivalence of categories between stacks which are faithful over $\mathfrak{X}$ and sheaves over $\mathfrak{X}$.

We define the global section functor

$$
\Gamma(\mathfrak{X}, \cdot):(\text { sheaves } / \mathfrak{X}) \longrightarrow(\text { sets })
$$

by $\Gamma(\mathfrak{X}, F)=\operatorname{Hom}_{\mathfrak{X}}(\mathfrak{X}, F)$, the set of morphisms of sheaves over $\mathfrak{X}$ from the trivial sheaf $\mathfrak{X}$ (whose set of sections is always the one point set $\{*\})$ to the sheaf $F$.

Remark 3.2 If $\mathfrak{X}$ is differentiable, $X \rightarrow \mathfrak{X}$ a presentation and $X_{1} \rightrightarrows X$ the associated Lie groupoid, then for any sheaf $F$ on $\mathfrak{X}$ we have a short exact sequence of sets

$$
\Gamma(\mathfrak{X}, F) \longrightarrow F(X) \Longrightarrow F\left(X_{1}\right) .
$$

In other words $\Gamma(\mathfrak{X}, F)$ is the equalizer of the two restriction maps $F(X) \rightarrow F\left(X_{1}\right)$. (Note that there are two canonical morphisms $X_{1} \rightarrow \mathfrak{X}$, so that $F\left(X_{1}\right)$ is ambiguous notation. But since both morphisms $X_{1} \rightarrow \mathfrak{X}$ are canonically isomorphic, it is irrelevant which choice one makes for $F\left(X_{1}\right)$.)

Restricting $\Gamma(\mathfrak{X}, \cdot)$ to the category of sheaves of abelian groups over $\mathfrak{X}$ we get the functor

$$
\Gamma(\mathfrak{X}, \cdot):(\text { abelian sheaves } / \mathfrak{X}) \longrightarrow(\text { abelian groups }) .
$$

This functor is left exact, and (abelian sheaves/X) has sufficiently many injectives, so we may derive this functor to get the functors

$$
\left.H^{i}(\mathfrak{X}, \cdot): \text { (abelian sheaves } / \mathfrak{X}\right) \longrightarrow \text { (abelian groups) } .
$$

Passing to the derived category of complexes of abelian sheaves over $\mathfrak{X}$, we get the total derived functor

$$
R \Gamma(\mathfrak{X}, \cdot): D^{+}(\mathfrak{X}) \longrightarrow D^{+} \text {(abelian groups). }
$$

For a complex $M^{\bullet} \in D^{+}(\mathfrak{X})$ of abelian sheaves on $\mathfrak{X}$, the homology groups of the complex $R \Gamma\left(\mathfrak{X}, M^{\bullet}\right)$ are denoted by

$$
\mathbb{H}^{i}\left(\mathfrak{X}, M^{\bullet}\right)=h^{i}\left(R \Gamma\left(\mathfrak{X}, M^{\bullet}\right)\right)
$$

and called the hypercohomology groups of $\mathfrak{X}$ with values in $M^{\bullet}$.

Of course, if $M^{\bullet} \rightarrow N^{\bullet}$ is a quasi-isomorphism of complexes of abelian sheaves over $\mathfrak{X}$, we get induced isomorphisms $\mathbb{H}^{i}\left(\mathfrak{X}, M^{\bullet}\right) \rightarrow \mathbb{H}^{i}\left(\mathfrak{X}, N^{\bullet}\right)$ on hypercohomology. 
Definition 3.3 Let $U$ be a manifold. A sheaf in the usual sense (defined only on open subsets of $U)$ is called a small sheaf on $U$. This is to distinguish such sheaves from sheaves over the stack over $\mathfrak{S}$ obtained from $U$.

Let $\mathfrak{X}$ be a stack over $\mathfrak{S}$ and $F$ a sheaf over $\mathfrak{X}$. Let $x$ be an object of $\mathfrak{X}$ lying over the manifold $U \in \mathfrak{S}$. The small sheaf on $U$, which maps the open subset $V \subset U$ to $F(x \mid V)$ is called the small sheaf induced by $F$ via $x: U \rightarrow \mathfrak{X}$ on $U$. Notation: $F_{x, U}$, or simply $F_{U}$, if there is no risk of confusion. Given a morphism $\theta: y \rightarrow x$ in $\mathfrak{X}$ lying over $f: V \rightarrow U$ in $\mathfrak{S}$, there is an induced morphism of small sheaves over $V$ called $\theta^{*}: f^{-1} F_{x, U} \longrightarrow F_{y, V}$. This induced morphism is contravariantly functorial in $\theta$.

Lemma 3.4 If $\mathfrak{X}$ is representable, represented by the manifold $X$, then for any big sheaf $F$ over $\mathfrak{X}$, we have $H^{i}(\mathfrak{X}, F)=H^{i}\left(X, F_{X}\right)$, for all $i$.

Proof. This follows from the fact that for a manifold $\mathfrak{X}=X$ the functor $F \mapsto F_{X}$, which maps a big sheaf to its associated small sheaf is exact. This property fails for stacks.

\subsection{Differential forms}

Let $\mathfrak{X}$ be a differentiable stack. Define the sheaf $\Omega_{\mathfrak{X}}^{i}$ of differential forms of degree $i$ on $\mathfrak{X}$ as follows: for an object $x \in \mathfrak{X}$ lying over $U \in \mathfrak{S}$, we let $\Omega_{\mathfrak{X}}^{i}(x)=\Omega^{i}(U)$ be the $\mathbb{R}$-vector space of (R्R-valued) differentiable $i$-forms on $U$. For a morphism $y \rightarrow x$ in $\mathfrak{X}$ lying over the $C^{\infty}$-map $V \rightarrow U$, we define the restriction map $\Omega_{\mathfrak{X}}^{i}(x) \rightarrow \Omega_{\mathfrak{X}}^{i}(y)$ to be the pullback map $\Omega^{i}(U) \rightarrow \Omega^{i}(V)$. The sheaf axioms are easily verified. Note that the $\Omega_{\mathfrak{X}}^{i}$ are sheaves of $\mathbb{R}$-vector spaces, i.e. they take values in $(\mathbb{R}$-vector spaces $) \subset$ (sets).

The sheaf $\Omega_{\mathfrak{X}}^{0}$ is also called the structure sheaf of $\mathfrak{X}$, notation $\mathcal{O}_{\mathfrak{X}}$. It is isomorphic to the sheaf of sections of the projection $\mathfrak{X} \times \mathbb{R} \rightarrow \mathfrak{X}$.

Note that none of the $\Omega_{\mathfrak{X}}^{i}$, for $i>0$, are isomorphic to sheaves of sections of any morphism of differentiable stacks $\mathfrak{Y} \rightarrow \mathfrak{X}$.

The exterior derivative $d: \Omega^{i}(U) \rightarrow \Omega^{i+1}(U)$, where $U$ is any manifold, commutes with the pullback of forms via any $C^{\infty}$-map $V \rightarrow U$. Thus $d$ induces a homomorphism of sheaves $d: \Omega_{\mathfrak{X}}^{i} \rightarrow$ $\Omega_{\mathfrak{X}}^{i+1}$, for all $i \geq 0$. Clearly, $d^{2}=0$, and so we have defined a complex $\Omega_{\mathfrak{X}}$ of sheaves of $\mathbb{R}$-vector spaces over $\mathfrak{X}$. We call $\Omega_{\mathfrak{X}}$ the de Rham complex of $\mathfrak{X}$. Its hypercohomology is called the de Rham cohomology of $\mathfrak{X}$ :

$$
H_{D R}^{i}(\mathfrak{X})=\mathbb{H}^{i}\left(\mathfrak{X}, \Omega_{\mathfrak{X}}\right) .
$$

If there is any danger of confusion (for example if $\mathfrak{X}$ is a manifold), we refer to $\Omega_{\mathfrak{X}}$ as the big de Rham complex of $\mathfrak{X}$.

Let $\mathbb{R}_{\mathfrak{X}}$ denote the sheaf over $\mathfrak{X}$ defined by

$$
\mathbb{R}_{\mathfrak{X}}(x)=\{f: U \rightarrow \mathbb{R} \mid f \text { is locally constant }\},
$$

for any object $x$ of $\mathfrak{X}$ lying over $U \in \mathfrak{S}$. The sheaf $\mathbb{R}_{\mathfrak{X}}$ is in a natural way a subsheaf of the structure sheaf $\Omega_{\mathfrak{X}}^{0}$. If we let $\widetilde{\mathbb{R}}$ denote the manifold with the same underlying set as $\mathbb{R}$, but the discrete differentiable structure, then we may identify $\mathbb{R}_{\mathfrak{X}}$ with the sheaf of sections of the projection $\mathfrak{X} \times \widetilde{\mathbb{R}} \rightarrow \mathfrak{X}$.

The usual Poincaré Lemma proves that the big de Rham complex $\Omega_{\mathfrak{X}}^{\bullet}$ is a resolution of $\mathbb{R}_{\mathfrak{X}}$. Thus we conclude that

$$
H_{D R}^{i}(\mathfrak{X})=H^{i}\left(\mathfrak{X}, \mathbb{R}_{\mathfrak{X}}\right),
$$

for all $i$. 


\subsection{Groupoid cohomology}

Let $\mathfrak{X}$ be a differentiable stack over $\mathfrak{S}$, and $X \rightarrow \mathfrak{X}$ an atlas for $\mathfrak{X}$. Define for all $p \geq 0$

$$
X_{p}=\underbrace{X \times \mathfrak{X} \ldots \times_{\mathfrak{X}} X}_{p+1 \text { times }} .
$$

(hence $X_{0}=X$ ). Since $X \rightarrow \mathfrak{X}$ is a representable submersion, all $X_{p}$ are manifolds. Of course, $X_{1} \rightrightarrows X_{0}$ is the Lie groupoid associated to the atlas $X \rightarrow \mathfrak{X}$. Furthermore, we assume that $X_{1} \rightrightarrows X_{0}$ is a Hausdorff and second countable Lie groupoid [28, 38].

We have $p+1$ projection maps $X_{p} \rightarrow X_{p-1}$, giving rise to a diagram

$$
\cdots \underset{\exists}{\rightrightarrows} X_{2} \rightleftarrows X_{1} \rightleftarrows
$$

In fact more is true: $X_{\bullet}$ is a simplicial manifold.

Every $X_{p}$ has $p+1$ canonical projections $X_{p} \rightarrow \mathfrak{X}$. They are all canonically isomorphic to each other. Choose any one of them and call it $\pi_{p}: X_{p} \rightarrow \mathfrak{X}$. As usual, we identify $\pi_{p}$ with an object of $\mathfrak{X}$ lying over $X_{p}$. Let $F$ be a sheaf over $\mathfrak{X}$. We denote the set $F\left(\pi_{p}\right)$ by $F\left(X_{p}\right)$. Let $F_{p}$ denote the small sheaf on $X_{p}$ induced by $F$. Then we have $F\left(X_{p}\right)=\Gamma\left(X_{p}, F_{p}\right)$.

Diagram (8) induces a diagram

$$
F\left(X_{0}\right) \Longrightarrow F\left(X_{1}\right) \rightleftarrows F\left(X_{2}\right) \stackrel{\rightrightarrows}{\rightleftarrows} \cdots
$$

which can, in fact, be refined to a cosimplicial set.

Now assume that $F$ is a sheaf of abelian groups. Let $\partial: F\left(X_{p}\right) \rightarrow F\left(X_{p+1}\right)$ be the alternating sum of the maps of Diagram (9). We obtain a complex of abelian groups

$$
F\left(X_{0}\right) \stackrel{\partial}{\longrightarrow} F\left(X_{1}\right) \stackrel{\partial}{\longrightarrow} F\left(X_{2}\right) \stackrel{\partial}{\longrightarrow} \cdots
$$

The homology groups of this complex are denoted by

$$
\check{H}^{i}\left(X_{\bullet}, F\right)=h^{i}\left(F\left(X_{\bullet}\right)\right)
$$

and called the $\check{C}$ ech cohomology groups of $F$ with respect to the covering $X \rightarrow \mathfrak{X}$.

Note that when $F$ is the sheaf $\Omega^{0}, \check{H}^{i}\left(X_{\bullet}, \Omega^{0}\right)$ is also called groupoid cohomology with trivial coefficients $[28,49]$.

Lemma 3.4 gives us the following lemma and proposition.

Lemma 3.5 There is an $E_{1}$ spectral sequence

$$
H^{q}\left(X_{p}, F_{p}\right) \Longrightarrow H^{p+q}(\mathfrak{X}, F) .
$$

Proposition 3.6 Assume that for every $p$ the induced small sheaf $F_{p}$ is acyclic, i.e. satisfies $H^{i}\left(X_{p}, F_{p}\right)=0$, for all $i>0$. Then we have

$$
\check{H}^{i}\left(X_{\bullet}, F\right)=H^{i}(\mathfrak{X}, F) .
$$

Corollary 3.7 We have, for all $i, j \geq 0$,

$$
\check{H}^{j}\left(X_{\bullet}, \Omega_{\mathfrak{X}}^{i}\right)=H^{j}\left(\mathfrak{X}, \Omega^{i}\right) .
$$


In particular we see that the sheaf cohomology $H^{j}\left(\mathfrak{X}, \Omega^{0}\right)$ is isomorphic to the groupoid cohomology.

In the sequel, when $X_{\bullet}$ is a Lie groupoid, a sheaf over $X_{\bullet}$ is defined to be a sheaf over the associated stack $\mathfrak{X}$. Moreover, we define groupoid cohomology $H^{i}(X ., F)$ to be equal to $H^{i}(\mathfrak{X}, F)$. This is in line with sheaf cohomology of simplicial manifolds.

Now let $M \bullet$ be a complex of abelian sheaves over $\mathfrak{X}$, bounded below. Denote the differential on $M^{\bullet}$ by $d$. Let $X \rightarrow \mathfrak{X}$ be an atlas. For every $i$ we get a Cech complex $M^{i}\left(X_{\bullet}\right)$, with differential $\partial$. Because $d$ and $\partial$ commute, we obtain, in fact, a double complex

$$
\left(\left\{M^{i}\left(X_{p}\right)\right\}_{i, p}, d, \partial\right) \text {. }
$$

Our convention will always be that $\partial$ is the vertical differential, $d$ the horizontal differential. The homology groups of the associated total complex are denoted by

$$
\check{\mathbb{H}}^{i}\left(X_{\bullet}, M^{\bullet}\right)=h^{i}\left(\operatorname{tot} M^{\bullet}\left(X_{\bullet}\right)\right)
$$

and called the Čech hypercohomology groups of $M \bullet$ with respect to the covering $X \rightarrow \mathfrak{X}$.

Proposition 3.8 Assume that for every $i$ and every $p$ the small sheaf $M_{p}^{i}$ induced by $M^{i}$ on $X_{p}$ is acyclic. Then we have

$$
\check{\mathbb{H}}^{i}\left(X_{\bullet}, M^{\bullet}\right)=\mathbb{H}^{i}\left(\mathfrak{X}, M^{\bullet}\right),
$$

for all $i \geq 0$.

Corollary 3.9 We have, for every atlas $X \rightarrow \mathfrak{X}$,

$$
H_{D R}^{i}(\mathfrak{X})=\check{\mathbb{H}}^{i}\left(X_{\bullet}, \Omega_{\mathfrak{X}}\right)=h^{i}\left(\operatorname{tot} \Omega^{\bullet}\left(X_{\bullet}\right)\right) .
$$

In the sequel, we also use $H_{D R}^{i}\left(X_{\bullet}\right)$ to denote the above cohomology group. We also write $\Omega^{k}\left(X_{\bullet}\right):=\oplus_{i+j=k} \Omega^{i}\left(X_{j}\right), Z^{k}\left(X_{\bullet}\right)$ and $B^{k}\left(X_{\bullet}\right)$ to denote the spaces of $k$-cochains, $k$-cocycles and $k$-coboundaries of tot $\Omega^{\bullet}\left(X_{\bullet}\right)$, respectively.

In particular, if $\mathfrak{X}$ is representable, represented by a manifold $X$, then $H_{D R}^{i}(\mathfrak{X})$ coincides with the $i$-th homology group of the usual de Rham complex of $X$. Thus our definition of de Rham cohomology gives the usual de Rham cohomology in the case of manifolds.

Remark The wedge product of differential forms turns $\Omega_{\mathfrak{X}}^{\bullet}$ into a sheaf of differential graded $\mathbb{R}$ algebras. From general principles it follows that $R \Gamma\left(\mathfrak{X}, \Omega^{\bullet}\right)$ is also a differential graded $\mathbb{R}$-algebra. Thus the hypercohomology $\mathbb{H}^{*}\left(\mathfrak{X}, \Omega^{\bullet}\right)$ is a graded $\mathbb{R}$-algebra.

This multiplicative structure can be described explicitly at the level of the double complex $\Omega^{\bullet}\left(X_{\bullet}\right)$ associated to an atlas $X \rightarrow \mathfrak{X}$ of $\mathfrak{X}$.

Let $a \in \Omega^{k}\left(X_{p}\right)$ and $b \in \Omega^{l}\left(X_{q}\right)$. Define $a \cup b \in \Omega^{k+l}\left(X_{p+q}\right)$ by

$$
a \cup b=(-1)^{k q} p_{1}^{*} a \wedge p_{2}^{*} b,
$$

where $p_{1}, p_{2}$ are the natural projections from $X_{p+q}$ to $X_{p}$ and $X_{q}$ given by $\left(x_{1}, \cdots, x_{p}, x_{p+1}, \cdots, x_{p+q}\right) \rightarrow\left(x_{1}, \cdots, x_{p}\right)$ and $\left(x_{1}, \cdots, x_{p}, x_{p+1}, \cdots, x_{p+q}\right) \rightarrow\left(x_{p+1}, \cdots, x_{p+q}\right)$ respectively. One checks that for any $a, b, c \in \Omega^{\bullet}\left(X_{\bullet}\right)$ the following identities hold:

$$
\begin{aligned}
& (a \cup b) \cup c=a \cup(b \cup c) \\
& \delta(a \cup b)=\delta a \cup b+(-1)^{|a|} a \cup \delta b,
\end{aligned}
$$

where $\delta$ is the total differential of the double complex $\Omega^{\bullet}\left(X_{\bullet}\right)$, and $|a|$ denotes the total degree of $a$ in the double complex $\Omega^{\bullet}\left(X_{\bullet}\right)$. Thus $\left(\Omega^{\bullet}\left(X_{\bullet}\right), \cup, \delta\right)$ is a graded differential algebra. One can prove that the induced graded algebra structure on its cohomology groups coincides with the one on $\mathbb{H}^{*}\left(\mathfrak{X}, \Omega^{\bullet}\right)$. 


\section{$4 \quad S^{1}$-bundles and $S^{1}$-gerbes}

In this section we study connections on bundles and gerbes. We often restrict to the case of $S^{1}$ as structure group.

\section{1 $\quad S^{1}$-bundles}

In this subsection, we study differential geometry, including characteristic classes, of $S^{1}$-bundles over a differentiable stack in terms of Lie groupoids.

Let $\mathfrak{X}$ be a differentiable stack and $X_{1} \rightrightarrows X_{0}$ a Lie groupoid presenting $\mathfrak{X}$. By an $X_{\bullet}$-space, we mean a manifold $P_{0} \rightarrow X_{0}$ with a left $X_{\bullet}$-action.

Definition 4.1 An $S^{1}$-bundle over $\mathfrak{X}$ is a 2-commutative diagram

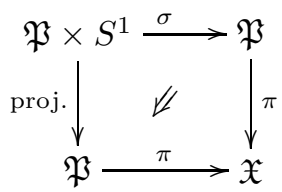

such that the pullback via $U \rightarrow \mathfrak{X}$, for any submersion from a manifold $U$, defines an $S^{1}$-bundle over $U$. (Note that this implies that $\mathfrak{P} \rightarrow \mathfrak{X}$ is a representable surjective submersion, and hence that $\mathfrak{P} \mid U$ is an $S^{1}$-bundle for every $U \rightarrow \mathfrak{X}$, submersive or not.)

Definition 4.2 Let $X_{1} \rightrightarrows X_{0}$ be a Lie groupoid. A (right) $S^{1}$-bundle over $X_{1} \rightrightarrows X_{0}$ is a (right) $S^{1}$-bundle $P_{0}$ over $X_{0}$, together with a (left) action of $X_{\bullet}$ on $P_{0}$, which respects the $S^{1}$-action, i.e. we have

$$
(\gamma \cdot x) \cdot t=\gamma \cdot(x \cdot t)
$$

for all $t \in S^{1}$ and all compatible pairs $(\gamma, x) \in \Gamma \times_{t, X_{0}} P_{0}$.

Proposition 4.3 There is a canonical equivalence of categories

$$
\left(S^{1} \text {-bundles over } \mathfrak{X}\right) \longrightarrow\left(S^{1} \text {-bundles over } X_{1} \rightrightarrows X_{0}\right) \text {. }
$$

Proof. Let $\mathfrak{P} \rightarrow \mathfrak{X}$ be an $S^{1}$-bundle. Denote the pullback of $X_{0}$ via $\mathfrak{P} \rightarrow \mathfrak{X}$ by $P_{0}$. Thus $P_{0} \rightarrow X_{0}$ is an $S^{1}$-bundle by assumption. And $P_{0} \rightarrow \mathfrak{P}$ is a representable submersion. Let $P_{1} \rightrightarrows P_{0}$ be the associated groupoid. We get an induced morphism of groupoids $P_{\bullet} \rightarrow X_{\bullet}$, which is cartesian, i.e. the diagram

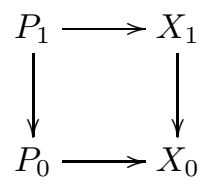

is a pullback diagram of manifolds, where the vertical maps are source maps (or, equivalently, target maps). Therefore $P_{1} \rightarrow X_{1}$ is an $S^{1}$-bundle and the vertical maps in the diagram above are $S^{1}$-bundle maps. As a consequence, $X_{1}$ acts on $P_{0}$ and Eq. (13) is satisfied. The functor in the proposition is $\mathfrak{P} \mapsto P_{0}$.

Conversely, given an $S^{1}$-bundle $P_{0}$ over $X_{1} \rightrightarrows X_{0}$, let $P_{1}=X_{1} \times_{t, X_{0}} P_{0}$. Action and projection form a diagram $P_{1} \rightrightarrows P_{0}$, and it is easy to check that $P_{1} \rightrightarrows P_{0}$ is naturally a groupoid (called the transformation groupoid of the $X_{1}$-action). It is clear that $P_{1}$ is an $S^{1}$-bundle over $X_{1}$. Moreover, 
there is a natural morphism of groupoids $\pi$ from $P_{1} \rightrightarrows P_{0}$ to $X_{1} \rightrightarrows X_{0}$, which respects the $S^{1}$ bundle structures $P_{1} \rightarrow X_{1}$ and $P_{0} \rightarrow X_{0}$. Let $\mathfrak{P}$ be the corresponding stack of $P_{\bullet}$-torsors. The groupoid morphism $P_{\bullet} \rightarrow X$. induces a morphism of stacks $\mathfrak{P} \rightarrow \mathfrak{X}$, which is representable, as its pullback to $X_{0}$ equals $P_{0} \rightarrow X_{0}$. It is also simple to see that for any morphism $U \rightarrow \mathfrak{X}, \mathfrak{P} \mid U$ is an $S^{1}$-bundle. Therefore $\mathfrak{P}$ is an $S^{1}$-bundle over $\mathfrak{X}$. The backwards functor is given by $P_{0} \mapsto \mathfrak{P}$.

As a consequence, $S^{1}$-bundles over a given Lie groupoid $X_{1} \rightrightarrows X_{0}$ are classified by $H^{1}\left(X_{\bullet}, S^{1}\right)$. The exponential sequence $\mathbb{Z} \rightarrow \Omega^{0} \rightarrow S^{1}$ induces a boundary map $H^{1}\left(X_{\bullet}, S^{1}\right) \rightarrow H^{2}\left(X_{\bullet}, \mathbb{Z}\right)$; the image of the class of an $S^{1}$-bundle under this boundary map is called its Chern class.

Let $P_{0} \rightarrow X_{0}$ be a principal $S^{1}$-bundle over $X_{1} \rightrightarrows X_{0}$. Let $\theta \in \Omega^{1}\left(P_{0}\right)$ be a connection 1-form on $P_{0}$. One checks that $\delta \theta \in \Omega_{D R}^{2}\left(P_{\bullet}\right)$ descends to $\Omega_{D R}^{2}\left(X_{\bullet}\right)$. In other words, there exist unique $\omega \in \Omega^{1}\left(X_{1}\right)$ and $\Omega \in \Omega^{2}\left(X_{0}\right)$ such that $\pi^{*}(\omega+\Omega)=\delta \theta$.

Proposition 4.4 The class $[\omega+\Omega] \in H_{D R}^{2}\left(X_{\bullet}\right)$ is independent of the choice of the connection $\theta$ on $P_{0} \rightarrow X_{0}$. Under the canonical homomorphism $H^{2}\left(X_{\bullet}, \mathbb{Z}\right) \rightarrow H^{2}\left(X_{\bullet}, \mathbb{R}\right) \cong H_{D R}^{2}\left(X_{\bullet}\right)$, the Chern class of $P$ maps to $[\omega+\Omega]$.

Proof. The proof of independence of choice of connection is a direct calculation. See [26]. Thus we concentrate on the second statement.

Let $\mathfrak{X}$ be the differentiable stack represented by $X$. and $\mathfrak{P}$ the $S^{1}$-bundle on $\mathfrak{X}$ defined by $P_{\bullet}$. Consider on $\mathfrak{X}$ the diagram of abelian sheaves

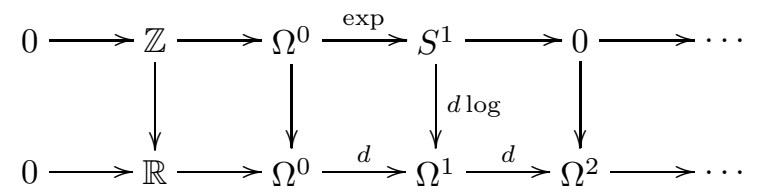

The upper row is a resolution $\left[\Omega^{0} \rightarrow S^{1}\right]$ of $\mathbb{Z}$, and the lower row is the de Rham resolution $\Omega^{\bullet}$ of $\mathbb{R}$, and the whole diagram is a morphism of resolutions.

It follows that we have a commutative diagram

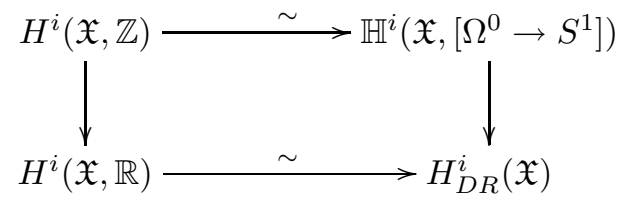

This diagram gives us a way to calculate the image of the Chern class of $\mathfrak{P}$ in de Rham cohomology.

In fact, consider now the Čech resolution (simplicial manifold) $X$. For any complex of sheaves $F^{\bullet}$, we get an associated double complex of abelian groups $F^{\bullet}\left(X_{\bullet}\right)$ and canonical maps

$$
h^{i}\left(\operatorname{tot} F^{\bullet}\left(X_{\bullet}\right)\right) \longrightarrow \mathbb{H}^{i}\left(\mathfrak{X}, F^{\bullet}\right) .
$$

(If all $F^{q}$ are acyclic on every $X_{p}$, then these are isomorphisms.)

If we assume that $P_{0}$ admits a section $\sigma$ over $X_{0}$, then $\rho=t^{*}(\sigma)-s^{*}(\sigma) \in S^{1}\left(X_{1}\right)$ is a 2-cocycle in $\operatorname{tot}\left[\Omega^{0} \rightarrow S^{1}\right]\left(X_{\bullet}\right)$ and the associated cohomology class $[\rho] \in \mathbb{H}^{2}\left(\mathfrak{X},\left[\Omega \rightarrow S^{1}\right]\right)$ is the image of the Chern class of $\mathfrak{P}$ under the upper row of (15).

The morphism of complexes of sheaves (14) induces a morphism of double complexes of abelian groups

$$
\left[\Omega^{0} \rightarrow S^{1}\right]\left(X_{\bullet}\right) \longrightarrow \Omega^{\bullet}\left(X^{\bullet}\right)
$$


The image of $\rho$ under this morphism is

$$
d \log \left(t^{*}(\sigma)-s^{*}(\sigma)\right) \in \Omega^{1}\left(X_{1}\right) .
$$

Now, $\sigma$ also induces a flat connection on the $S^{1}$-bundle $P_{0} \rightarrow X_{0}$ (ignoring the groupoid action), hence a connection 1-form $\theta \in \Omega^{1}\left(P_{0}\right)$. We have $\delta(\theta)=s^{*}(\theta)-t^{*}(\theta)$, and thus, all we need to prove is that

$$
s^{*}(\theta)-t^{*}(\theta)=\pi^{*}\left(d \log \left(t^{*} \sigma-s^{*} \sigma\right)\right) .
$$

This can be easily checked.

Let us now prove the general case, i.e. the case where $P_{0}$ is not necessarily trivial over $X_{0}$.

Choose an open covering $\left(U_{i}\right)$ of $X_{0}$ such that each $U_{i}$ is contractible. Let $Y_{0}=\coprod U_{i}$ and $Y_{1}=\coprod U_{i} \times_{s} X_{1} \times_{t} U_{j}$. Then $Y_{1} \rightrightarrows Y_{0}$ is a Lie groupoid Morita equivalent to $X_{1} \rightrightarrows X_{0}$. In fact, the projection $f: Y_{\bullet} \rightarrow X_{\bullet}$ is a Morita morphism. Let $Q_{\bullet}$ be the $S^{1}$-bundle over $Y_{\bullet}$ induced from $P$. Since $Q_{0}$ can be trivialized over $Y_{0}$, we choose $\left[\omega^{\prime}+\Omega^{\prime}\right]$ coming from a trivialization of $Q_{0}$, as above (in which case $\Omega^{\prime}=0$ ).

Choose $(\omega, \Omega)$ for $P_{\bullet}$ as in the statement of the proposition. By Morita invariance of de Rham cohomology of Lie groupoids, to prove that $[\omega+\Omega]$ is the Chern class of $\mathfrak{P}$, it suffices to do this for $f^{*}[\omega+\Omega]$. Thus we reduce to proving that $f^{*}[\omega+\Omega]=\left[\omega^{\prime}+\Omega^{\prime}\right] \in H_{D R}^{2}\left(Y_{\bullet}\right)$, which is just the invariance under choice of connection.

Note that the class $[\omega+\Omega]$ in the above proposition is an integer class in $H_{D R}^{2}\left(X_{\bullet}\right)$, and $\omega+\Omega$ an integer 2-cocycle in $Z_{D R}^{2}\left(X_{\bullet}\right)$. In general a $k$-cocycle in $Z_{D R}^{k}\left(X_{\bullet}\right)$ is said to be an integer $k$ cocycle if it defines an integer class in $H_{D R}^{k}\left(X_{\bullet}\right)$, i.e. a class in the image of the homomorphism $H^{k}\left(X_{\bullet}, \mathbb{Z}\right) \rightarrow H^{k}\left(X_{\bullet}, \mathbb{R}\right) \cong H_{D R}^{k}\left(X_{\bullet}\right)$.

Definition 4.5 A connection 1-form $\theta$ on $P_{0}$ is called a pseudo-connection on $P_{\text {. }}$. The de Rham cocycle $\omega+\Omega \in Z_{D R}^{2}\left(X_{\bullet}\right)$ such that $\pi^{*}(\omega+\Omega)=\delta \theta$ is called the pseudo-curvature of $\theta$.

A pseudo-connection $\theta$ is a connection if $\partial \theta=0$.

A flat connection is a pseudo-connection $\theta$ whose pseudo-curvature vanishes.

Remark Unlike in the manifold case, connections do not always exist. Thus connections are not necessarily as useful to compute characteristic classes as in the manifold case. For instance, the universal $S^{1}$-bundle $* \rightarrow B S^{1}$, which corresponds to $S^{1} \rightarrow *$ considered as an $S^{1}$-bundle over the groupoid $S^{1} \rightrightarrows *$ (where the groupoid $S^{1} \rightrightarrows *$ acts on $S^{1}$ by left translation), does not admit any connections. (Any connection on the universal bundle would necessarily be flat, and the existence of a flat connection on the universal bundle would imply that all connections on all bundles over all manifolds were flat.)

A flat $S^{1}$-bundle is an $S^{1}$-bundle with a flat connection. It is simple to see that a flat $S^{1}$-bundle over $X_{\bullet}$ is equivalent to a $\mathbb{R} / \mathbb{Z}$-bundle over $X_{\bullet}$. Therefore, the equivalence classes of flat $S^{1}$-bundles are classified by $H^{1}\left(X_{\bullet}, \mathbb{R} / \mathbb{Z}\right)$. The functor

$$
\left\{\text { flat } S^{1} \text {-bundles over } X_{\bullet}\right\} \longrightarrow H^{1}\left(X_{\bullet}, \mathbb{R} / \mathbb{Z}\right)
$$

is called the holonomy map. When $X$. is a manifold, this reduces to the usual holonomy map for flat bundles.

We are now ready to prove the following proposition, which generalizes the prequantization theorem of Kostant and Weil [24, 48]. 
Proposition 4.6 Assume that $\check{H}^{1}\left(X_{\bullet}, \Omega^{0}\right)=0$. Let $\omega+\Omega \in Z_{D R}^{2}\left(X_{\bullet}\right)$ be an integer 2-cocycle. Then there exists an $S^{1}$-bundle $P$. over $X_{1} \rightrightarrows X_{0}$ and a pseudo-connection $\theta$ whose pseudocurvature is $\omega+\Omega$.

Moreover, the set of isomorphism classes of all such pairs $\left(P_{\bullet}, \theta\right)$ is a simply transitive $H^{1}\left(X_{\bullet}, \mathbb{R} / \mathbb{Z}\right)$-set. Here $\left(P_{\bullet}, \theta\right)$ and $\left(P_{\bullet}^{\prime}, \theta^{\prime}\right)$ are isomorphic if $P_{1}$ and $P_{1}^{\prime}$ are isomorphic as $S^{1}$ bundles over $X_{1} \rightrightarrows X_{0}$ and under such an isomorphism $\theta$ is identified with $\theta^{\prime}$.

Proof. Consider the exact sequence

$$
\rightarrow H^{1}\left(X_{\bullet}, S^{1}\right) \stackrel{\phi}{\rightarrow} H^{2}\left(X_{\bullet}, \mathbb{Z}\right) \rightarrow H^{2}\left(X_{\bullet}, \Omega^{0}\right) \rightarrow
$$

induced by the exponential sequence $\mathbb{Z} \rightarrow \Omega^{0} \rightarrow S^{1}$. The map $H^{2}\left(X_{\bullet}, \mathbb{Z}\right) \rightarrow H^{2}\left(X_{\bullet}, \Omega^{0}\right)$ factors through $H^{2}\left(X_{\bullet}, \mathbb{R}\right) \cong H_{D R}^{2}\left(X_{\bullet}\right)$, i.e. we have the following commutative diagram

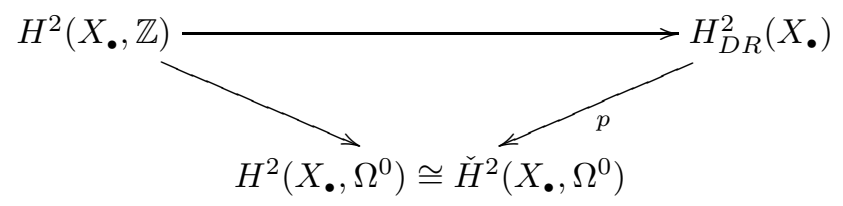

where $p$ is the natural projection. It is clear that $p([\omega+\Omega])=0$. Thus there is an $S^{1}$-bundle $P$. over $X_{1} \rightrightarrows X_{0}$, whose Chern class equals $[\omega+\Omega]$. Let $\theta^{\prime} \in \Omega^{1}\left(P_{0}\right)$ be a pseudo-connection, and $\delta \theta^{\prime}=\pi^{*}\left(\omega^{\prime}+\Omega^{\prime}\right)$. According to Proposition 4.4, $\omega+\Omega$ and $\omega^{\prime}+\Omega^{\prime}$ are cohomologous. Hence, $\omega+\Omega-\left(\omega^{\prime}+\Omega^{\prime}\right)=\delta(f+\alpha)$ for some $f \in \Omega^{0}\left(X_{1}\right)$ and $\alpha \in \Omega^{1}\left(X_{0}\right)$. It thus follows that $\partial f=0$, which implies that $f=\partial g$ for $g \in \Omega^{0}\left(X_{0}\right)$ since $\check{H}^{1}\left(X_{\bullet}, \Omega^{0}\right)=0$. Thus $\delta f=\delta \partial g=\delta d g$. Let $\theta=\theta^{\prime}+\pi^{*}(\alpha+d g) \in \Omega^{1}\left(P_{0}\right)$. It is clear that $\theta$ is the desired pseudo-connection.

If $(P, \theta)$ and $\left(P^{\prime}, \theta^{\prime}\right)$ are two such $S^{1}$-bundles, then $\left(P \otimes \overline{P^{\prime}}, \operatorname{pr}_{1}^{*} \theta+\operatorname{pr}_{2}^{*} \overline{\theta^{\prime}}\right)$ is a flat bundle, whose isomorphism class is classified by $H^{1}(X, \mathbb{R} / \mathbb{Z})$. Here $P \otimes \overline{P^{\prime}}$ denotes the $S^{1}$-bundle $\left(P \times_{X_{0}} P^{\prime}\right) / S^{1}$, and $\mathrm{pr}_{1}: P \otimes \overline{P^{\prime}} \rightarrow P$ and $\mathrm{pr}_{2}: P \otimes \overline{P^{\prime}} \rightarrow P^{\prime}$ are projections. This completes the proof.

Remark The condition $\check{H}^{1}\left(X_{\bullet}, \Omega^{0}\right)=0$ always holds for a proper Lie groupoid $X_{1} \rightrightarrows X_{0}$ according to Crainic [10]. In particular, when $X_{1} \rightrightarrows X_{0}$ is a manifold $M \rightrightarrows M$ (which is clearly a proper Lie groupoid), an integer 2-cocycle in $Z_{D R}^{2}\left(X_{\bullet}\right)$ corresponds to an integer closed two-form on $M$. Thus Proposition 4.6 reduces to the usual prequantization theorem of Kostant and Weil $[24,48]$.

\section{2 $\quad S^{1}$-gerbes and $S^{1}$-central extensions}

Let us first recall the definition of gerbes. Let $\mathfrak{X}$ be the differentiable stack associated to the Lie groupoid $X_{1} \rightrightarrows X_{0}$. Thus $\mathfrak{X}$ is the stack of $X_{\bullet}$-torsors.

Definition 4.7 An $\mathfrak{S}$-stack $\mathfrak{R}$, endowed with a morphism $\mathfrak{R} \rightarrow \mathfrak{X}$ is called a gerbe over $\mathfrak{X}$, if both $\mathfrak{R} \rightarrow \mathfrak{X}$ and $\mathfrak{R} \rightarrow \mathfrak{R} \times \mathfrak{X} \mathfrak{R}$ are epimorphisms.

Remark Under the correspondence between $\mathfrak{S}$-stacks equipped with morphisms to $\mathfrak{X}$ and $\mathfrak{X}$ stacks, the gerbes over $\mathfrak{X}$, according to our definition, correspond to gerbes over the site $\mathfrak{X}$ in the usual sense, i.e. in the sense of Giraud [19], Chapter III.2.

$B S^{1} \times \mathfrak{X} \rightarrow \mathfrak{X}$ is an example of a gerbe over $\mathfrak{X}$. We will study gerbes that locally look like this example.

The groupoid of automorphisms of $B S^{1}$ is equal to the transformation groupoid of $S^{1}$ on Aut $S^{1} \cong \mathbb{Z}_{2}$. This action is by "inner automorphisms" and hence trivial, as $S^{1}$ is abelian. The 
group of automorphism classes of $B S^{1}$ is therefore equal to $\mathbb{Z}_{2}$. The sheaf of automorphism classes of $B S^{1} \times \mathfrak{X}$ over $\mathfrak{X}$, which takes $U / \mathfrak{X}$ to the 2 -isomorphism classes of diagrams

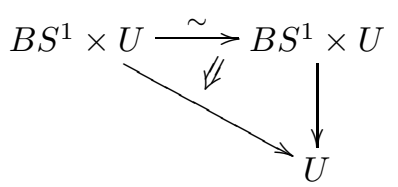

is therefore equal to $\mathbb{Z}_{2} \times \mathfrak{X} \rightarrow \mathfrak{X}$. So if the gerbe $\mathfrak{R} \rightarrow \mathfrak{X}$ is locally isomorphic to $B S^{1} \times \mathfrak{X}$, then the sheaf of automorphism classes of $\mathfrak{R}$ over $\mathfrak{X}$, which maps $U / \mathfrak{X}$ to the 2 -isomorphism classes of diagrams

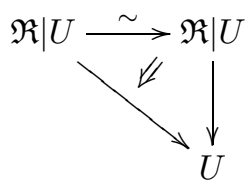

is a 2 -sheeted covering $\underline{B \text { and }}(\mathfrak{R}) \rightarrow \mathfrak{X}$, called the band of $\mathfrak{R}$.

Definition 4.8 An $S^{1}$-gerbe over $\mathfrak{X}$ is a gerbe $\mathfrak{R} \rightarrow \mathfrak{X}$ which is locally isomorphic to $B S^{1} \times \mathfrak{X}$ and is endowed with a trivialization of its band (the 2 -sheeted covering $\underline{\operatorname{Band}}(\mathfrak{R}) \rightarrow \mathfrak{X})$.

The following is a well-known theorem of Giraud [19].

Theorem 4.9 (Giraud) Isomorphism classes of $S^{1}$-gerbes over $\mathfrak{X}$ are in one-to-one correspondence with $H^{2}\left(\mathfrak{X}, S^{1}\right)$.

Now we recall $S^{1}$-central extensions of Lie groupoids [49].

Definition 4.10 Let $X_{1} \rightrightarrows X_{0}$ be a Lie groupoid. An $S^{1}$-central extension of $X_{1} \rightrightarrows X_{0}$ consists of

1. a Lie groupoid $R_{1} \rightrightarrows X_{0}$, together with a morphism of Lie groupoids $(\pi$, id $):\left[R_{1} \rightrightarrows X_{0}\right] \rightarrow$ $\left[X_{1} \rightrightarrows X_{0}\right]$

2. a left $S^{1}$-action on $R_{1}$, making $\pi: R_{1} \rightarrow X_{1}$ a (left) principal $S^{1}$-bundle.

These two structures are compatible in the sense that $(s \cdot x)(t \cdot y)=s t \cdot(x y)$, for all $s, t \in S^{1}$ and $(x, y) \in R_{1} \times_{X_{0}} R_{1}$.

The proposition below gives an equivalent definition.

Proposition 4.11 Let $X_{1} \rightrightarrows X_{0}$ be a Lie groupoid. A Lie groupoid $R_{1} \rightrightarrows X_{0}$ is an $S^{1}$-central extension of $X_{1} \rightrightarrows X_{0}$ if and only if it is endowed with $\phi$ and $\pi$ forming an exact sequences of groupoid morphisms

$$
1 \rightarrow X_{0} \times S^{1} \stackrel{\phi}{\rightarrow} R_{1} \stackrel{\pi}{\rightarrow} X_{1} \rightarrow 1
$$

over the identities on the unit spaces, and the image of $\phi$ lies in the center of $R_{1}$.

PROof. The proof is straightforward and is left to the reader.

The following result describes the precise connection between $S^{1}$-gerbes and $S^{1}$-central extensions. 
Proposition 4.12 Let $X_{1} \rightrightarrows X_{0}$ be a Lie groupoid and $\mathfrak{X}$ its corresponding differentiable stack of $X .-$ torsors. There is a one-to-one correspondence between isomorphism classes of $S^{1}$-central extensions of $X_{1} \rightrightarrows X_{0}$ and isomorphism classes of $S^{1}$-gerbes $\mathfrak{R}$ over $\mathfrak{X}$ endowed with a trivialization of the restriction of $\mathfrak{R}$ to $X_{0}$.

Proof. Given an $S^{1}$-central extension $R_{1} \rightrightarrows X_{0}$ of $X_{1} \rightrightarrows X_{0}$, let $\mathfrak{R}$ be the stack of $R_{\bullet}$-torsors and $\mathfrak{X}$ the stack of $X_{\bullet}$-torsors. Then the groupoid morphism $\pi$ induces a morphism of stacks $\mathfrak{R} \rightarrow \mathfrak{X}$, via which we think of $\mathfrak{R}$ as a stack over $\mathfrak{X}$.

The groupoid morphism $S^{1} \times X_{0} \stackrel{\phi}{\rightarrow} R_{1}$ induces the morphism of stacks $B S^{1} \times X_{0} \rightarrow \mathfrak{R}$. Consider the diagram

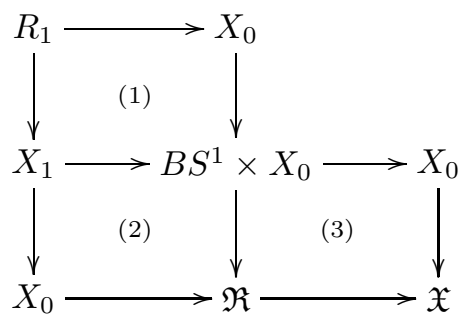

The square (1) is cartesian, because $R_{1} \mid X_{1}$ is an $S^{1}$-torsor. The combination of squares (1) and (2) is cartesian by definition of $\Re$. Hence, by descent, (2) is cartesian. The combination of squares (2) and (3) is cartesian by definition of $\mathfrak{X}$, and so, again by descent, square (3) is cartesian. This proves that $\mathfrak{R}$ restricted to $X_{0}$ is isomorphic to $B S^{1} \times X_{0}$, and in particular, $\mathfrak{R} \rightarrow \mathfrak{X}$ satisfies the first condition in the definition of $S^{1}$-gerbe.

The band of $\mathfrak{R} \rightarrow \mathfrak{X}$ is an Out $\left(S^{1}\right)$-torsor, trivialized by $X_{0}$, so the band is given by a map $X_{1} \rightarrow \operatorname{Out}\left(S^{1}\right)$. It is given as follows: $x \mapsto\left[A d_{\widetilde{x}}\right], \forall x \in X_{1}$, where $\widetilde{x} \in R_{1}$ is any point satisfying $\pi(\widetilde{x})=x$ and $A d_{\widetilde{x}} y=\widetilde{x} y \widetilde{x}^{-1}$. Here $y \in \operatorname{ker} \pi_{x} \cong S^{1}$. Then because $\operatorname{ker} \pi$ is central in $R_{1}$, the map $X_{1} \rightarrow \operatorname{Out}\left(S^{1}\right)$ is trivial, showing that the band of $\mathfrak{R}$ is trivial.

Conversely, given such a gerbe $\mathfrak{R}$, by taking the section $X_{0} \rightarrow \mathfrak{R} \mid X_{0}$, one obtains a commutative diagram of stacks:

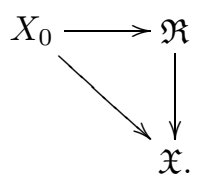

So $X_{0} \rightarrow \mathfrak{R}$ is a presentation. Let $R_{1}=X_{0} \times_{\mathfrak{R}} X_{0}$. Thus we have a Lie groupoid morphism $(\pi$, id $):\left[R_{1} \rightrightarrows X_{0}\right] \rightarrow\left[X_{1} \rightrightarrows X_{0}\right]$. Moreover the kernel of $\pi$ is isomorphic to $X_{0} \times S^{1}$ as a bundle of groups, by assumption. Since Band $\mathfrak{R}$ is trivial, it follows that the conjugation action of $R_{1}$ on $\operatorname{ker} \pi$ must be trivial. Therefore $\operatorname{ker} \pi$ lies in the center of $R_{1}$. This concludes the proof.

\subsection{Morita equivalence of $S^{1}$-central extensions}

We now introduce the definition of Morita equivalence of $S^{1}$-central extensions.

Definition 4.13 We say that two $S^{1}$-central extensions $R_{1} \rightarrow X_{1} \rightrightarrows X_{0}$ and $R_{1}^{\prime} \rightarrow X_{1}^{\prime} \rightrightarrows X_{0}^{\prime}$ are Morita equivalent if there exists an $S^{1}$-equivariant $R_{\bullet}-R_{\bullet}^{\prime}$-bitorsor $Z$, by which we mean that $Z$ is an $R_{\bullet}$ - $R_{\bullet}^{\prime}$-bitorsor endowed with an $S^{1}$-action such that

$$
(\lambda r) \cdot z \cdot r^{\prime}=r \cdot(\lambda z) \cdot r^{\prime}=r \cdot z \cdot\left(\lambda r^{\prime}\right)
$$

whenever $\left(\lambda, r, r^{\prime}, z\right) \in S^{1} \times R \times R^{\prime} \times Z$ and the products make sense. 
The following result is immediate.

Lemma 4.14 Let $R_{1} \rightarrow X_{1} \rightrightarrows X_{0}$ and $R_{1}^{\prime} \rightarrow X_{1}^{\prime} \rightrightarrows X_{0}^{\prime}$ be Morita equivalent $S^{1}$-central extensions, and $Z$ an $S^{1}$-equivariant $R_{\bullet}-R_{\bullet}^{\prime}$-bitorsor. Then the $S^{1}$-action on $Z$ must be free and $Z / S^{1}$ is a $X_{\bullet}-X_{\bullet}^{\prime}$-bitorsor. As a consequence, $X_{\bullet}$ and $X_{\bullet}^{\prime}$ are Morita equivalent.

Proposition 4.15 Let $R_{1} \rightarrow X_{1} \rightrightarrows X_{0}$ and $R_{1}^{\prime} \rightarrow X_{1}^{\prime} \rightrightarrows X_{0}^{\prime}$ be $S^{1}$-central extensions of Lie groupoids. Let $\mathfrak{R}, \mathfrak{R}^{\prime}, \mathfrak{X}$, and $\mathfrak{X}^{\prime}$ be their associated stacks. Then the following are equivalent:

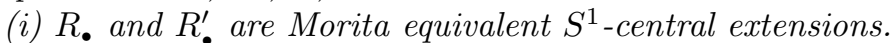

(ii) there exists an $S^{1}$-central extension $R_{1}^{\prime \prime} \rightarrow X_{1}^{\prime \prime} \rightrightarrows X_{0}^{\prime \prime}$ and $S^{1}$-equivariant Morita morphisms $R_{\bullet}^{\prime \prime} \rightarrow R_{\bullet}^{\prime}$ and $R_{\bullet}^{\prime \prime} \rightarrow R_{\bullet}$.

(iii) $\mathfrak{X} \cong \mathfrak{X}^{\prime}$, over which $\mathfrak{R} \cong \mathfrak{R}^{\prime}$ as $S^{1}$-gerbes.

Proof. To prove that (i) implies (ii), choose an $S^{1}$-equivariant $R_{\bullet}-R_{\bullet}^{\prime}$-bitorsor $Z$. Take $R_{1}^{\prime \prime}=$ $R_{1} \times_{X_{0}} Z \times_{X_{0}^{\prime}} R_{1}^{\prime}$ and $X_{1}^{\prime \prime}=X_{1} \times_{X_{0}} X \times_{X_{0}^{\prime}} X_{1}^{\prime}$, where $X=Z / S^{1}$. Then it is simple to see that $R_{1}^{\prime \prime} \rightarrow X_{1}^{\prime \prime} \rightrightarrows X$ is the desired $S^{1}$-central extension.

For (ii) to imply (iii), assuming that $R_{1}^{\prime \prime} \rightarrow X_{1}^{\prime \prime} \rightrightarrows X_{0}^{\prime \prime}$ is such an $S^{1}$-central extension, using Theorem 2.26, one has the commutative diagram

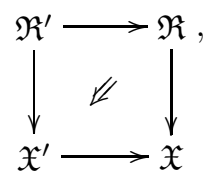

where the horizontal maps are isomorphism of stacks. (iii) thus follows.

Finally, we prove that (iii) implies (i). By identifying $\mathfrak{X}$ with $\mathfrak{X}^{\prime}$ and $\mathfrak{R}$ with $\mathfrak{R}^{\prime}$, we may think $R_{1} \rightarrow X_{1} \rightrightarrows X_{0}$ and $R_{1}^{\prime} \rightarrow X_{1}^{\prime} \rightrightarrows X_{0}^{\prime}$ as the $S^{1}$-central extensions corresponding to the presentations $X_{0} \rightarrow \mathfrak{R}$ and $X_{0}^{\prime} \rightarrow \mathfrak{R}$ respectively. Take $Z=X_{0} \times_{\mathfrak{R}} X_{0}^{\prime}$. Then $Z$ is an $S^{1}$ equivariant $R_{\bullet}-R_{\bullet}^{\prime}$-bitorsor.

We end this subsection by the following exact sequences:

Proposition 4.16 There is a natural exact sequence

$$
\begin{aligned}
& H^{1}\left(X_{\bullet}, S^{1}\right) \stackrel{\tau_{1}}{\longrightarrow} H^{1}\left(X_{0}, S^{1}\right) \stackrel{\tau_{2}}{\longrightarrow} \\
&\left\{S^{1} \text {-central extensions of } X_{1} \rightrightarrows X_{0}\right\} \stackrel{\tau_{3}}{\longrightarrow} H^{2}\left(X_{\bullet}, S^{1}\right) \stackrel{\tau_{4}}{\longrightarrow} H^{2}\left(X_{0}, S^{1}\right) .
\end{aligned}
$$

Proof. Let $\mathfrak{X}$ be the stack of $X_{\bullet}$-torsors. Note that $\tau_{1}, \cdots, \tau_{4}$ can be geometrically described as follows:

1. $\tau_{1}$ is the map sending an $S^{1}$-bundle $L \rightarrow \mathfrak{X}$ to its restriction to $X_{0}$, i.e. to $L \rightarrow X_{0}$ by forgetting the groupoid $X_{1} \rightrightarrows X_{0}$-action.

2. $\tau_{2}$ sends an $S^{1}$-bundle $L \rightarrow X_{0}$ to the $S^{1}$-central extension $\left(s^{*} L \times_{X_{1}} t^{*} L^{-1}\right) / S^{1} \rightarrow X_{1} \rightrightarrows X_{0}$. In stack language, $\tau_{2}$ maps the $S^{1}$-bundle $L$ to the stack of descent data (gluing data) for $L$ over the groupoid $X_{\bullet}$.

3. $\tau_{3}$ sends an $S^{1}$-central extension to the class in $H^{2}\left(X_{\bullet}, S^{1}\right)$ of its corresponding gerbe.

4. $\tau_{4}$ is the pull back map under the map $X_{0} \rightarrow \mathfrak{X}$. 
Let $\phi: L \rightarrow X_{0}$ be an $S^{1}$-bundle over $X_{1} \rightrightarrows X_{0}$. Define a map $X_{1} \times S^{1} \rightarrow\left(s^{*} L \times_{X_{1}} t^{*} \bar{L}\right) / S^{1}$ by $(r, \lambda) \rightarrow\left[\left((r l) \lambda^{-1}, l\right)\right]$, where $l \in L$ is any point satisfying $\phi(l)=t(r)$. One checks that this is an isomorphism of $S^{1}$-central extensions. Conversely, if $\phi: L \rightarrow X_{0}$ is an $S^{1}$-bundle over $X_{0}$ such that $\left(s^{*} L \times_{X_{1}} t^{*} \bar{L}\right) / S^{1}$ is a trivial central extension, then $\left(s^{*} L \times_{X_{1}} t^{*} \bar{L}\right) / S^{1} \rightarrow X_{1}$ admits a section $\sigma$ which is a groupoid homomorphism. Then the equation $\sigma(r)=[(r \cdot l, l)]$, where $l \in L$ such that $\phi(l)=t(r)$, defines a groupoid action of $X_{1} \rightrightarrows X_{0}$ on $L$. This shows that the sequence is exact at $H^{1}\left(X_{0}, S^{1}\right)$.

Let $R$ be the $S^{1}$-central extension $\left(s^{*} L \times_{X_{1}} t^{*} \bar{L}\right) / S^{1}$, where $L \rightarrow X_{0}$ is an $S^{1}$-bundle. One checks that the pullback groupoid $R[L] \rightrightarrows L$ is a trivial extension of $X_{1}[L] \rightrightarrows L$, which means that $R$ defines the zero class in $H^{2}\left(X_{\bullet}, S^{1}\right)$. Conversely, if $R$ is an $S^{1}$-central extension defining the zero class in $H^{2}\left(X_{\bullet}, S^{1}\right)$, then $R$ is Morita equivariant to the trivial central extension $X_{1} \times$ $S^{1} \rightrightarrows X_{0}$ via an $S^{1}$-equivariant bimodule $Y$ : then $L=Y / X_{1} \stackrel{\rho}{\rightarrow} X_{0}$ is an $S^{1}$-bundle. One checks easily that $R$ is isomorphic to $\left(s^{*} L \times_{X_{1}} t^{*} \bar{L}\right) / S^{1}$. This shows that the sequence is exact at $\left\{S^{1}\right.$-central extensions of $\left.X_{1} \rightrightarrows X_{0}\right\}$.

Finally the exactness at $H^{2}\left(X_{\bullet}, S^{1}\right)$ follows from Theorem 4.9 and Proposition 4.12.

\subsection{Dixmier-Douady classes}

Let $R$ be an $S^{1}$-central extension of $X_{1} \rightrightarrows X_{0}$. Write the underlying Lie groupoid of $R$ as $R_{1} \rightrightarrows R_{0}$. Call the structure morphism $\pi: R_{\bullet} \rightarrow X_{\bullet}$. Since $R_{1} \rightrightarrows R_{0}$ defines an $S^{1}$-gerbe over $\mathfrak{X}$, it defines a class in $H^{2}\left(X_{\bullet}, S^{1}\right)$ according to Theorem 4.9. The exponential sequence gives rise to a homomorphism $H^{2}\left(X_{\bullet}, S^{1}\right) \rightarrow H^{3}\left(X_{\bullet}, \mathbb{Z}\right)$. The image of $[R] \in H^{2}\left(X_{\bullet}, S^{1}\right)$ in $H^{3}\left(X_{\bullet}, \mathbb{Z}\right)$ is called the Dixmier-Douady class of $R$ and denoted by $D D(R)$. The Dixmier-Douady class behaves well with respect to pullbacks and the tensor operation.

Let $f: Y_{\bullet} \rightarrow X$ • be a Lie groupoid homomorphism. Then the pullback $S^{1}$-bundle $f^{*} R_{1} \rightarrow Y_{1}$ is an $S^{1}$-central extension over $Y_{1} \rightrightarrows Y_{0}$, called the pullback central extension.

Assume that $R^{\prime}$ and $R^{\prime \prime}$ are two $S^{1}$-central extensions of $X_{1} \rightrightarrows X_{0}$. Let $R_{1}=\left(R^{\prime} \times_{X_{1}} R^{\prime \prime}\right) / S^{1}$, where $S^{1}$ acts on $R^{\prime} \times_{X_{1}} R^{\prime \prime}$ by $t \cdot\left(r_{1}, r_{2}\right)=\left(t \cdot r_{1}, t^{-1} \cdot r_{2}\right), \forall t \in S^{1}, \quad\left(r_{1}, r_{2}\right) \in R^{\prime} \times_{X_{1}} R^{\prime \prime}$. It is clear that $R_{1}$ with the natural projection to $X_{1}$ is still an $S^{1}$-principal bundle, where the $S^{1}$-action is given by $t \cdot\left[\left(r_{1}, r_{2}\right)\right]=\left[\left(t \cdot r_{1}, r_{2}\right)\right]$. The groupoid structures on $R^{\prime}$ and $R^{\prime \prime}$ induce a groupoid structure on $R_{1}$ in a natural way, which in fact makes $R_{1}$ into a groupoid $S^{1}$-central extension, called the tensor product of $R^{\prime}$ and $R^{\prime \prime}$ and is denoted, by $R^{\prime} \otimes R^{\prime \prime}$.

The following proposition can be easily verified.

\section{Proposition 4.17 1. $D D\left(f^{*} R\right)=f^{*} D D(R)$; and}

$$
\text { 2. } D D\left(R^{\prime} \otimes R^{\prime \prime}\right)=D D\left(R^{\prime}\right)+D D\left(R^{\prime \prime}\right) \text {. }
$$

Definition 4.18 Let $\theta \in \Omega^{1}\left(R_{1}\right)$ be a connection 1 -form for the $S^{1}$-principal bundle $R_{1} \rightarrow X_{1}$, and $B \in \Omega^{2}\left(R_{0}\right)$ be any 2 -form. Any such pair $(\theta, B)$ is called a pseudo-connection for the central extension $R$.

It is simple to check that $\delta(\theta+B) \in Z_{D R}^{3}\left(R_{\bullet}\right)$ descends to $Z_{D R}^{3}\left(X_{\bullet}\right)$, i.e. there exist unique $\eta \in \Omega^{1}\left(X_{2}\right), \omega \in \Omega^{2}\left(X_{1}\right)$ and $\Omega \in \Omega^{3}\left(X_{0}\right)$ such that

$$
\delta(\theta+B)=\pi^{*}(\eta+\omega+\Omega) .
$$

Then $\eta+\omega+\Omega$ is called the pseudo-curvature of the pseudo-connection $\theta+B$.

We will now show that pseudo-connections can be used to calculate Dixmier-Douady classes. 
Theorem 4.19 The class $[\eta+\omega+\Omega] \in H_{D R}^{3}\left(X_{\bullet}\right)$ is independent of the choice of the pseudoconnection $\theta+B$. Under the canonical homomorphism $H^{3}\left(X_{\bullet}, \mathbb{Z}\right) \rightarrow H_{D R}^{3}\left(X_{\bullet}\right)$, the DixmierDouady class of $R$ maps to $[\eta+\omega+\Omega]$.

Proof. One checks directly that the class $[\eta+\omega+\Omega] \in H_{D R}^{3}\left(X_{\bullet}\right)$ is independent of the choice of the pseudo-connection $\theta+B$.

We prove the second part of the theorem. Let $\mathfrak{X}$ be the stack given by $X_{1} \rightrightarrows X_{0}$ and $\mathfrak{R} \rightarrow \mathfrak{X}$ the $S^{1}$-gerbe over $\mathfrak{X}$ defined by $R_{1} \rightrightarrows R_{0}$.

We will construct a hypercovering in the site $\mathfrak{X}$. Note that $R$ • is a simplicial object in $\mathfrak{X}$. The hypercovering we shall use is the 1-coskeleton of $R_{\bullet}$ :

$$
Y_{\bullet}=\operatorname{cosk}_{\mathfrak{X}} R_{\bullet}
$$

This is a hypercovering because $R_{1} \rightarrow R_{0} \times \mathfrak{X} R_{0}=X_{1}$ and $R_{0} \rightarrow \mathfrak{X}$ are surjective submersions. (For the theory of hypercoverings, see $[1,2,12]$. In the generality we need them, the necessary results are proved in [1].)

Intuitively, $Y_{\bullet}$ is the set of all (1-skeleta of) simplices in $R_{\bullet}$, whose image in $X$. commutes. Explicitly, $Y_{p}$ is the fibered product

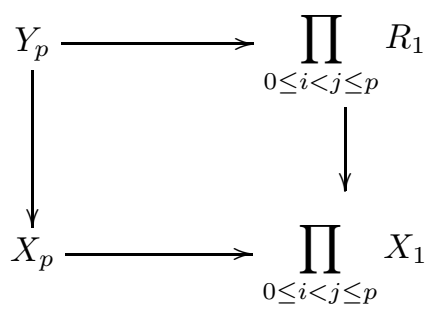

Here the horizontal arrow at the bottom is the map which sends $X_{p}$ to the edges of a commutative $p$-simplex, i.e. the product of $\left(\begin{array}{c}p+1 \\ 2\end{array}\right)$ maps $f_{i j}: X_{p} \rightarrow X_{1}, 0 \leq i<j \leq p$

$$
\left(x_{1}, x_{2}, \cdots, x_{p}\right) \rightarrow x_{i+1} \cdots x_{j} .
$$

Since $Y_{\bullet}$ is a hypercovering of $\mathfrak{X}$, we have a canonical homomorphism

$$
f: \check{H}^{2}\left(Y_{\bullet}, S^{1}\right) \longrightarrow H^{2}\left(\mathfrak{X}, S^{1}\right) .
$$

Since $\Omega^{\bullet}$ consists of soft sheaves, we also have an isomorphism

$$
\check{H}^{2}\left(Y_{\bullet}, \Omega^{\bullet}\right) \stackrel{\sim}{\longrightarrow} H^{2}\left(\mathfrak{X}, \Omega^{\bullet}\right) .
$$

We will see that the class of $\mathfrak{R}$ in $H^{2}\left(\mathfrak{X}, S^{1}\right)$ is in the image of the homomorphism (17).

In fact,

$$
Y_{2}=\left\{(\alpha, \beta, \gamma) \in R_{1} \times R_{1} \times R_{1} \mid \pi(\alpha) \pi(\gamma)=\pi(\beta)\right\},
$$

so we have a $C^{\infty}$-map

$$
\begin{aligned}
c: Y_{2} & \longrightarrow S^{1} \\
(\alpha, \beta, \gamma) & \longmapsto(\alpha \gamma) \beta^{-1} .
\end{aligned}
$$

Recall that a composition in $R_{1}$ makes sense if and only if the composition of its image in $X_{1}$ makes sense and that we have $\operatorname{ker} \pi=S^{1}$. One checks that the coboundary of $c$ vanishes, and so $c$ 
defines a Čech cohomology class $[c] \in \check{H}^{2}\left(Y_{\bullet}, S^{1}\right)$. It is simple to see that $f([c])$ is the cohomology class of $\mathfrak{R}$.

Now consider the diagram

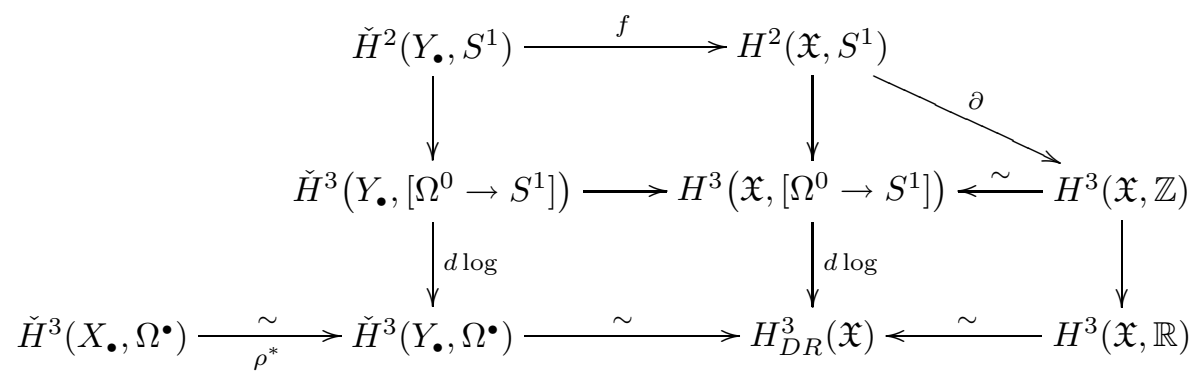

which commutes. The two vertical arrows in the first row are induced by the trivial map

$$
S^{1} \longrightarrow\left[\Omega^{0} \rightarrow S^{1}\right][1],
$$

i.e. the map

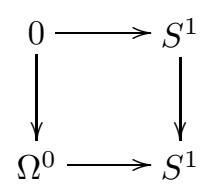

Considering this diagram, we see that we need to prove that

$$
d \log ([c])=\rho^{*}([\eta+\omega+\Omega]) \in \check{H}^{3}\left(Y_{\bullet}, \Omega^{\bullet}\right),
$$

where we have denoted the canonical projection by $\rho: Y_{\bullet} \rightarrow X_{\bullet}$ and its induced map on Cech cohomology by $\rho^{*}$. We have also committed the abuse of denoting $[c]$ and its induced class in $\check{H}^{3}\left(Y_{\bullet},\left[\Omega^{0} \rightarrow S^{1}\right]\right)$ by the same letter.

First we may assume that $B=0$ (thus $\Omega=0$ ) for simplicity since the class $[\eta+\omega+\Omega]$ is independent of the pseudo-connection. Thus we have

$$
\partial \theta=\pi^{*} \eta, \quad d \theta=-\pi^{*} \omega .
$$

We have the following commutative diagram:

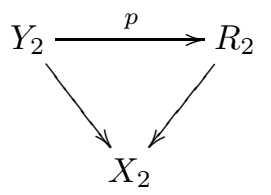

where $p: Y_{2} \rightarrow R_{2}$ is the natural projection. We have

$$
\rho^{*} \eta=p^{*} \pi^{*} \eta=p^{*} \partial \theta=\alpha^{*} \theta-(\alpha \gamma)^{*} \theta+\gamma^{*} \theta,
$$

where, by abuse of notation, we denote by $\alpha, \gamma$ and $\alpha \gamma$ the maps $Y_{2} \rightarrow Y_{1}$ sending $(\alpha, \beta, \gamma)$ to $\alpha, \gamma$ and $\alpha \gamma \in Y_{1}$, respectively.

Since $\rho=\pi$ on $Y_{1}$, we have $\rho^{*} \omega=\pi^{*} \omega=-d \theta \in \Omega^{2}\left(Y_{1}\right)$, which is cohomologous to $-\partial_{Y_{\bullet}} \theta$ in $\check{H}^{3}\left(Y_{\bullet}, \Omega^{\bullet}\right)$. The latter is equal to $-\left(\alpha^{*} \theta-\beta^{*} \theta+\gamma^{*} \theta\right) \in \Omega^{1}\left(Y_{2}\right)$. Thus it follows that

$$
\rho^{*}([\eta+\omega])=\beta^{*}\left[\theta-(\alpha \gamma)^{*} \theta\right] .
$$


Now it suffices to prove that

$$
d \log ([c])=\beta^{*} \theta-(\alpha \gamma)^{*} \theta \in \Omega^{1}\left(Y_{2}\right) .
$$

Let $\psi: R_{2} \times S^{1} \rightarrow Y_{2}$ be the diffeomorphism given by $(\alpha, \gamma, t) \rightarrow(\alpha, t(\alpha \gamma), \gamma)$. Then $\psi^{*}(d \log ([c]))$ is the Maurer-Cartan form $d t$ on $S^{1}$, while $\beta^{*} \theta-(\alpha \gamma)^{*} \theta$ is easily seen to be equal to $d t$ as well.

This completes the proof.

\subsection{Prequantization}

Definition 4.20 Given an $S^{1}$-central extension $R_{1} \rightarrow X_{1} \rightrightarrows X_{0}$,

(i) a connection 1-form $\theta \in \Omega^{1}\left(R_{1}\right)$ for the $S^{1}$-principal bundle $R_{1} \rightarrow X_{1}$, such that $\partial \theta=0$ is a connection;

(ii) Given $\theta$, a 2-form $B \in \Omega^{2}\left(X_{0}\right)$, such that $d \theta=\partial B$ is a curving;

(iii) and given $(\theta, B)$, the 3 -form $\Omega=d B \in H^{0}\left(X_{\bullet}, \Omega^{3}\right) \subset \Omega^{3}\left(X_{0}\right)$ is called the 3-curvature of $(\theta, B)$;

(iv) If $\Omega=0$, then $R_{1} \rightarrow X_{1} \rightrightarrows X_{0}$ together with $(\theta, B)$ is called a flat $S^{1}$-central extension of $X_{1} \rightrightarrows X_{0}$. Note that the flat central extensions form an abelian group.

In other words, a flat $S^{1}$-central extension of $X_{1} \rightrightarrows X_{0}$ is an $S^{1}$-central extension with a pseudo-connection whose pseudo-curvature vanishes. The following proposition is immediate.

Proposition 4.21 Let $R_{1} \rightarrow X_{1} \rightrightarrows X_{0}$ be an $S^{1}$-central extension. Then

(i) $H^{2}\left(X_{\bullet}, \Omega^{1}\right)$ contains the obstruction to the existence of a connection;

(ii) if we assume the existence of a connection, $H^{1}\left(X_{\bullet}, \Omega^{2}\right)$ contains the obstruction to the existence of a curving.

According to Theorem 4.19, we have the following

Proposition 4.22 If an $S^{1}$-central extension $R_{1} \rightarrow X_{1} \rightrightarrows X_{0}$ admits a connection and a curving with 3-curvature $\Omega$, then $[\Omega] \in H_{D R}^{3}\left(X_{\bullet}\right)$ is the image of its Dixmier-Douady class under the canonical homomorphism $H^{3}\left(X_{\bullet}, \mathbb{Z}\right) \rightarrow H_{D R}^{3}\left(X_{\bullet}\right)$.

Remark Given a manifold $M$, and a surjective submersion $X_{0} \rightarrow M, X_{1}\left(=X_{0} \times_{M} X_{0}\right) \rightrightarrows X_{0}$ is a Lie groupoid Morita equivalent to $M$. An $S^{1}$-central extension $R_{1} \rightarrow X_{1} \rightrightarrows X_{0}$ defines a bundle gerbe over $M$ in the terminology of Murray [32,33]. Since $\Omega^{1}$ and $\Omega^{2}$ are soft sheaves over $M$, we have $H^{2}\left(X_{\bullet}, \Omega^{1}\right) \cong H^{2}\left(M, \Omega^{1}\right)=0$ and $H^{1}\left(X_{\bullet}, \Omega^{2}\right) \cong H^{1}\left(M, \Omega^{2}\right)=0$. As a consequence, connections and curvings always exist for bundle gerbes. This result was due to Murray [32]. Moreover, in this case, the 3-curvature $\Omega \in \Omega^{3}\left(X_{0}\right)$ descends to a closed 3-form on $M$ since $\partial \Omega=0$.

In particular, for an open cover $\left\{U_{i}\right\}$ of $M$, one can take $X_{0}=\coprod U_{i}$. Then $X_{1} \cong \coprod U_{i j}$. An $S^{1}$-central extension $R_{1} \rightarrow X_{1} \rightrightarrows X_{0}$ corresponds in this case to a family of line bundles $L_{i j} \rightarrow U_{i j}$ satisfying all the axioms of bundle gerbes as in [21]. This is the case of Chatterjee-Hitchin bundle gerbes $[8,21]$

Proposition 4.23 Assume that $H^{2}\left(X_{0}, \mathbb{R}\right)=0$. There is a natural exact sequence

$$
\begin{aligned}
H^{1}\left(X_{\bullet}, \mathbb{R} / \mathbb{Z}\right) \longrightarrow & H^{1}\left(X_{0}, \mathbb{R} / \mathbb{Z}\right) \longrightarrow \\
& \left\{\text { flat } S^{1} \text {-central extensions of } X_{1} \rightrightarrows X_{0}\right\} \longrightarrow H^{2}\left(X_{\bullet}, \mathbb{R} / \mathbb{Z}\right) \longrightarrow H^{2}\left(X_{0}, \mathbb{R} / \mathbb{Z}\right) .
\end{aligned}
$$

The proof is similar to that of Proposition 4.16 via replacing $S^{1}$ by $\mathbb{R} / \mathbb{Z}$, and using the following 
Lemma 4.24 Let $X_{1} \rightrightarrows X_{0}$ be a Lie groupoid. Assume that $H^{2}\left(X_{0}, \mathbb{R}\right)=0$. Then there is a canonical one-to-one correspondence between flat $S^{1}$-central extensions of $X_{1} \rightrightarrows X_{0}$ and $\mathbb{R} / \mathbb{Z}$ central extensions of $X_{1} \rightrightarrows X_{0}$.

Proof. Let $\left(R_{1} \rightarrow X_{1} \rightrightarrows X_{0}, \theta, B\right)$ be a flat $S^{1}$-central extension. Then, in particular, $d B=0$. Since $H^{2}\left(X_{0}, \mathbb{R}\right)=0$, we can write $B=d A$, where $A \in \Omega^{1}\left(X_{0}\right)$. Set $\theta^{\prime}=\theta+\partial A \in \Omega^{1}\left(R_{1}\right)$. Then $\theta^{\prime}$ is again a connection 1-form for the principal $S^{1}$-bundle $R_{1} \rightarrow X_{1}$, which satisfies $d \theta^{\prime}=0$ and $\partial \theta^{\prime}=0$. The condition $d \theta^{\prime}=0$ implies that $R_{1} \rightarrow X_{1}$ is flat, and can therefore equivalently be considered as an $\mathbb{R} / \mathbb{Z}$-bundle. Moreover, $\partial \theta^{\prime}=0$ implies that under this new differentiable structure, $R_{1} \rightarrow X_{1}$ is still a smooth groupoid homomorphism, and therefore an $\mathbb{R} / \mathbb{Z}$-central extension.

Conversely, given an $\mathbb{R} / \mathbb{Z}$-central extension $R_{1} \rightarrow X_{1} \rightrightarrows X_{0}$, then $R_{1} \rightarrow X_{1}$ is a flat $S^{1}$-bundle. Let $\theta \in \Omega^{1}\left(R_{1}\right)$ be a flat connection one-form, i.e. $d \theta=0$. Locally, if we write $R_{1} \cong X_{1} \times \mathbb{R} / \mathbb{Z}$, then we may choose $\theta=d t$ where $t$ is the coordinate on $\mathbb{R} / \mathbb{Z}$. Moreover locally the groupoid multiplication on $R_{1}$ is written as

$$
(x, t) \cdot(y, s)=(x \cdot y, t+s+\omega(x, y)), \quad \forall(x, y) \in X_{2}, \quad t, s \in \mathbb{R} / \mathbb{Z} .
$$

It is easy to see that $\omega(x, y)$ must be locally constant. Therefore it follows that $\partial \theta=0$. Hence $R_{1} \rightarrow X_{1} \rightrightarrows X_{0}$ is a flat $S^{1}$-central extension.

Following Hitchin [21], we call the map

$$
\left\{\text { flat } S^{1} \text {-central extensions of } X_{1} \rightrightarrows X_{0}\right\} \longrightarrow H^{2}(X, \mathbb{R} / \mathbb{Z})
$$

the holonomy map.

Next we give the following prequantization result, which can be considered as an analogue, in the degree 3-context, of the well known theorem of Weil and Kostant [24, 48].

Theorem 4.25 Assume that $\check{H}^{2}\left(X_{\bullet}, \Omega^{0}\right)=0$. Given any 3-cocycle $\eta+\omega+\Omega \in Z_{D R}^{3}\left(X_{\bullet}\right)$ as above, satisfying

1. $\eta+\omega+\Omega$ is an integer 3-cocycle, and

2. $\Omega$ is exact,

there exists a groupoid $S^{1}$-central extension $R_{1} \rightrightarrows X_{0}$ of the groupoid $X_{1} \rightrightarrows X_{0}$ and a pseudoconnection $\theta+B$ such that its pseudo-curvature is $\eta+\omega+\Omega$. The pairs $(\theta, B)$ up to isomorphism form a simply transitive set under the group of flat $S^{1}$-central extensions.

Proof. Consider the exact sequence

$$
\rightarrow H^{2}\left(X_{\bullet}, S^{1}\right) \stackrel{\phi}{\rightarrow} H^{3}\left(X_{\bullet}, \mathbb{Z}\right) \rightarrow H^{3}\left(X_{\bullet}, \Omega^{0}\right) \rightarrow
$$

induced by the exponential sequence $\mathbb{Z} \rightarrow \Omega^{0} \rightarrow S^{1}$. Since we have the following commutative diagram

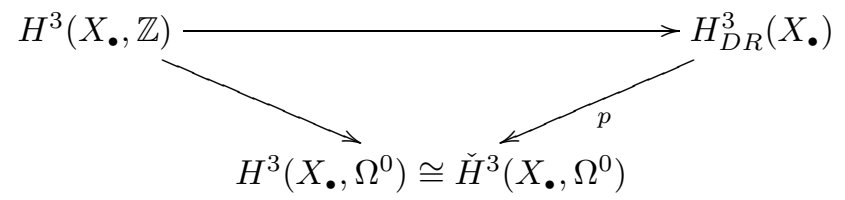

where $p$ is the natural projection, it is clear that $[\eta+\omega+\Omega]$ is in the image of $\phi$. Thus there is an $S^{1}$-gerbe $\mathfrak{R} \in H^{2}\left(X, S^{1}\right)$ whose Dixmier-Douady class equals $[\eta+\omega+\Omega]$. Note that the image 
of $\mathfrak{R}$ under the map $H^{2}\left(X_{\bullet}, S^{1}\right) \rightarrow H^{2}\left(X_{0}, S^{1}\right)$ is zero since $\Omega$ is exact. This follows from the commutative diagram

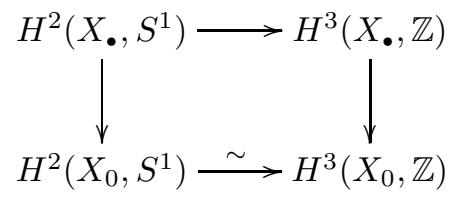

From Proposition 4.16 it follows that $\mathfrak{R}$ can be represented by an $S^{1}$-central extension $R_{1} \rightrightarrows R_{0}$ over $X_{1} \rightrightarrows X_{0}$, whose Dixmier-Douady class is $[\eta+\omega+\Omega]$.

Let $\theta^{\prime}+B^{\prime}$ be any pseudo-connection on the $S^{1}$-central extension $R_{1} \rightrightarrows R_{0}$ and $\eta^{\prime}+\omega^{\prime}+\Omega^{\prime}$ its pseudo-curvature. Proposition 4.19 implies that $\eta+\omega+\Omega$ and $\eta^{\prime}+\omega^{\prime}+\Omega^{\prime}$ are cohomologous. Therefore

$$
(\eta+\omega+\Omega)-\left(\eta^{\prime}+\omega^{\prime}+\Omega^{\prime}\right)=\delta\left(f+\alpha+B^{\prime \prime}\right),
$$

where $f \in \Omega^{0}\left(X_{2}\right), \alpha \in \Omega^{1}\left(X_{1}\right)$, and $B \in \Omega^{2}\left(X_{0}\right)$. It thus follows that $\partial f=0$, which implies that $f=\partial g$ for $g \in \Omega^{0}\left(X_{1}\right)$ since $\tilde{H}^{2}\left(X_{\bullet}, \Omega^{0}\right)=0$. Thus $\delta f=\delta \partial g=\delta d g$. Let $\theta=\theta^{\prime}+\pi^{*}(\alpha+d g) \in$ $\Omega^{1}\left(R_{1}\right)$ and $B=B^{\prime}+B^{\prime \prime}$. It is clear that $\theta+B$ is the desired pseudo-connection on $R_{1} \rightrightarrows R_{0}$. Finally note that if $\left(R^{\prime}, \theta^{\prime}, B^{\prime}\right)$ and $\left(R^{\prime \prime}, \theta^{\prime \prime}, B^{\prime \prime}\right)$ are two such pairs, then $\left(R^{\prime} \otimes\left(R^{\prime \prime}\right)^{-1}, \theta^{\prime}-\theta^{\prime \prime}, B^{\prime}-\right.$ $\left.B^{\prime \prime}\right)$ is a flat gerbe. So such pairs, up to isomorphism, are indeed parametrized by the group of flat $S^{1}$-central extensions.

Remark Note again, that the condition $\check{H}^{2}\left(X_{\bullet}, \Omega^{0}\right)=0$ always holds for a proper Lie groupoid $X_{1} \rightrightarrows X_{0}$, according to Crainic [10]. So prequantization always works for a proper Lie groupoids.

\section{$5 \quad S^{1}$-central extensions with prescribed pseudo-curvature}

\subsection{Geometry of $S^{1}$-central extensions}

First we need a technical lemma concerning $S^{1}$-principal bundles over a Lie groupoid (not necessary a groupoid central extension).

Let $X_{1} \rightrightarrows X_{0}$ be a Lie groupoid with a 3 -cocycle $\eta+\omega \in Z_{D R}^{3}\left(X_{\bullet}\right)$, where $\eta \in \Omega^{1}\left(X_{2}\right)$ and $\omega \in \Omega^{2}\left(X_{1}\right)$, and $R_{1} \stackrel{\pi}{\longrightarrow} X_{1}$ an $S^{1}$-principal bundle. Assume that $\theta \in \Omega^{1}\left(R_{1}\right)$ is a principal bundle connection one-form with curvature $-\omega$, i.e.

$$
d \theta=-\pi^{*} \omega
$$

Consider the $T^{2}$-action on $R_{1} \times R_{1} \times R_{1}$ :

$$
(s, t) \cdot(\widetilde{x}, \widetilde{y}, \widetilde{z})=(s \cdot \widetilde{x}, t \cdot \widetilde{y},(s t) \cdot \widetilde{z}), \quad \forall s, t \in S^{1}, \widetilde{x}, \widetilde{y}, \widetilde{z} \in R_{1} .
$$

Then $p:\left(R_{1} \times R_{1} \times R_{1}\right) / T^{2} \longrightarrow X_{1} \times X_{1} \times X_{1}$ is an $S^{1}$-principal bundle. Consider the following diagram of principal bundles

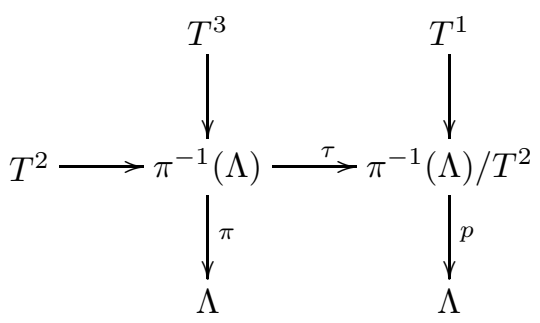


where $\Lambda=\left\{(x, y, z) \mid z=x y, \quad \forall(x, y) \in X_{2}\right\} \subset X_{1} \times X_{1} \times X_{1}$ is the graph of the groupoid multiplication of $X_{1} \rightrightarrows X_{0}$. Let $\widetilde{\Theta}$ be the one-form on $\pi^{-1}(\Lambda) \subset R_{1} \times R_{1} \times R_{1}$ defined by

$$
\widetilde{\Theta}=\Theta-\pi^{*} \operatorname{pr}_{12}^{*} \eta,
$$

where $\Theta=(\theta, \theta,-\theta)$ and $\operatorname{pr}_{12}: \Lambda \rightarrow X_{2}$ is the projection to the first two components. Then

$$
d \widetilde{\Theta}=0
$$

By $\xi$ we denote the Euler vector field on $R_{1}$ generating the $S^{1}$-action.

Lemma 5.1 (i). $(\xi, \xi, \xi)\lrcorner \widetilde{\Theta}=1$;

(ii). $\widetilde{\Theta} \in \Omega^{1}\left(\pi^{-1}(\Lambda)\right)$ is basic with respect to the $T^{2}$-action as in $E q$. (19), so it descends to a one-form $\widehat{\Theta}$ on $\pi^{-1}(\Lambda) / T^{2}$;

(iii). $\widehat{\Theta}$ defines a flat connection on the $S^{1}$-principal bundle $\pi^{-1}(\Lambda) / T^{2} \stackrel{p}{\longrightarrow} \Lambda$.

Proof. (i) is obvious. For (ii)-(iii), note that $\widetilde{\Theta} \in \Omega^{1}\left(\pi^{-1}(\Lambda)\right)$ is invariant under the natural $T^{3}$-action induced from the one on $R_{1} \times R_{1} \times R_{1}$. It is also quite clear that $\left.\left.\xi_{1}\right\lrcorner \widetilde{\Theta}=\xi_{2}\right\lrcorner \widetilde{\Theta}=0$, where

$$
\xi_{1}=(\xi, 0, \xi), \text { and } \xi_{2}=(0, \xi, \xi)
$$

are the generating vector fields of the $T^{2}$-action as in Eq. (19). Hence $\widetilde{\Theta}$ is basic with respect to this action, and descends to a one-form $\widehat{\Theta}$ on $\pi^{-1}(\Lambda) / T^{2}$, which is easily seen to be a flat connection for the $S^{1}$-bundle $\pi^{-1}(\Lambda) / T^{2} \stackrel{p}{\longrightarrow} \Lambda$.

Now assume that $R_{1} \rightrightarrows R_{0}$ is a Lie groupoid $S^{1}$-central extension over $X_{1} \rightrightarrows X_{0}$. Then $R_{1} \rightarrow X_{1}$ is a principal $S^{1}$-bundle. Assume, moreover, that $\theta \in \Omega^{1}\left(R_{1}\right)$ is a pseudo-connection of the extension whose corresponding pseudo-curvature equals $\eta+\omega \in Z_{D R}^{3}\left(X_{\bullet}\right)$. That is,

$$
\partial \theta=\pi^{*} \eta, \quad d \theta=-\pi^{*} \omega .
$$

The proposition below describes the relation between $\theta$ and the groupoid structure on $R_{1} \rightrightarrows R_{0}$. First, let us fix some notations as follows.

$$
\begin{aligned}
& \tilde{\epsilon}: R_{0} \longrightarrow R_{1}, \quad u \rightarrow \widetilde{u} \\
& \tilde{\epsilon}_{2}: R_{0} \longrightarrow R_{2}, \quad u \rightarrow(\widetilde{u}, \widetilde{u}) \\
& \epsilon_{2}: X_{0} \longrightarrow X_{2}, \quad u \rightarrow(u, u) \\
& \kappa: X_{1} \longrightarrow X_{2}, \quad x \longrightarrow\left(x, x^{-1}\right)
\end{aligned}
$$

Let $\eta_{0}$ be the one-form on $X_{0}$ given by

$$
\eta_{0}=\epsilon_{2}^{*} \eta
$$

Proposition 5.2 Let $R_{1} \rightrightarrows R_{0}$ be a Lie groupoid $S^{1}$-central extension over $X_{1} \rightrightarrows X_{0}$. Let $\theta \in \Omega^{1}\left(R_{1}\right)$ be a pseudo-connection whose corresponding pseudo-curvature equals $\eta+\omega \in Z_{D R}^{3}\left(X_{\bullet}\right)$. Then 
(i). $\widetilde{\epsilon}^{*} \theta=\eta_{0}$

(ii). $\widetilde{\iota}^{*} \theta+\theta=\widetilde{s}^{*} \eta_{0}+\pi^{*} \kappa^{*} \eta$.

In particular, if $\theta$ is a connection, then

$$
\widetilde{\epsilon}^{*} \theta=0, \quad \widetilde{\iota}^{*} \theta=-\theta .
$$

Proof. (i) It is clear that $\widetilde{\epsilon}_{2}^{*} \partial \theta=\widetilde{\epsilon}^{*} \theta$. On the other hand, we have $\widetilde{\epsilon}_{2}^{*} \pi^{*} \eta=\left(\pi_{\circ} \widetilde{\epsilon}_{2}\right)^{*} \eta=\epsilon_{2}^{*} \eta=\eta_{0}$. Thus we have $\widetilde{\epsilon}^{*} \theta=\eta_{0}$.

(ii) Given any $\widetilde{x} \in R_{1}, \forall \delta_{\widetilde{x}} \in T_{\widetilde{x}} R_{1}$, consider the tangent vector $\left(\delta_{\widetilde{x}}, \widetilde{\iota}_{*} \delta_{\widetilde{x}}\right)$ of $R_{2}$ at the point $\left(\widetilde{x}, \widetilde{x}^{-1}\right)$. It is clear that $\widetilde{m}_{*}\left(\delta_{\widetilde{x}}, \widetilde{\iota}_{*} \delta_{\widetilde{x}}\right)=\widetilde{\epsilon}_{*} \widetilde{s}_{*} \delta_{\widetilde{x}}$. So

$$
\left.\left.\left.\left.(\partial \theta)\left(\delta_{\widetilde{x}}, \widetilde{\iota}_{*} \delta_{\widetilde{x}}\right)=\delta_{\widetilde{x}}\right\lrcorner \theta+\widetilde{\iota}_{*} \delta_{\widetilde{x}}\right\lrcorner \theta-\widetilde{\epsilon}_{*} \widetilde{s}_{*} \delta_{\widetilde{x}}\right\lrcorner \theta=\delta_{\widetilde{x}}\right\lrcorner\left(\theta+\widetilde{\iota}^{*} \theta-\widetilde{s}^{*} \eta_{0}\right) .
$$

On the other hand, $\left.\left(\pi^{*} \eta\right)\left(\delta_{\widetilde{x}}, \widetilde{\iota}_{*} \delta_{\widetilde{x}}\right)=\delta_{\widetilde{x}}\right\lrcorner \pi^{*} \kappa^{*} \eta$. (ii) thus follows.

Remark 5.3 In the case of an $S^{1}$-gerbe over a manifold, the conditions that $\widetilde{\epsilon}^{*} \theta=0, \widetilde{\iota}^{*} \theta=-\theta$ were included in the definition of a connection [5, 21, 32]. From the above lemma, we see that they are easy consequences of the condition $\partial \theta=0$.

Proposition 5.4 Let $R_{1} \rightrightarrows R_{0}$ be a Lie groupoid $S^{1}$-central extension over $X_{1} \rightrightarrows X_{0}$. Let $\theta \in \Omega^{1}\left(R_{1}\right)$ be a pseudo-connection whose pseudo-curvature equals $\eta+\omega \in Z_{D R}^{3}\left(X_{\bullet}\right)$. Then the flat $S^{1}$-bundle $p: \pi^{-1}(\Lambda) / T^{2} \longrightarrow \Lambda$ as in diagram (20) is holonomy free.

Proof. By $\widetilde{\Lambda} \subseteq R_{1} \times R_{1} \times R_{1}$, we denote the graph of the groupoid multiplication of $R_{1} \rightrightarrows R_{0}$. It is clear that $p\left(\widetilde{\Lambda} / T^{2}\right)=\Lambda$. Given any $\left(\widetilde{x}_{1}, \widetilde{y}_{1}, \widetilde{z}_{1}\right),\left(\widetilde{x}_{2}, \widetilde{y}_{2}, \widetilde{z}_{2}\right) \in \widetilde{\Lambda}$, if $p\left[\left(\widetilde{x}_{1}, \widetilde{y}_{1}, \widetilde{z}_{1}\right)\right]=p\left[\left(\widetilde{x}_{2}, \widetilde{y}_{2}, \widetilde{z}_{2}\right)\right]$, then $\pi\left(\widetilde{x}_{1}, \widetilde{y}_{1}, \widetilde{z}_{1}\right)=\pi\left(\widetilde{x}_{2}, \widetilde{y}_{2}, \widetilde{z}_{2}\right)$. This implies that $\widetilde{x}_{1}=s \cdot \widetilde{x}_{2}$ and $\widetilde{y}_{1}=t \cdot \widetilde{y}_{2}$. Hence $\widetilde{z}_{1}=\widetilde{x}_{1} \widetilde{y}_{1}=$ $\left(s \cdot \widetilde{x}_{2}\right)\left(t \cdot \widetilde{y}_{2}\right)=(s t) \cdot\left(\widetilde{x}_{2} \widetilde{y}_{2}\right)=(s t) \cdot \widetilde{z}_{2}$, and therefore $\left[\left(\widetilde{x}_{1}, \widetilde{y}_{1}, \widetilde{z}_{1}\right)\right]=\left[\left(\widetilde{x}_{2}, \widetilde{y}_{2}, \widetilde{z}_{2}\right)\right]$. Hence $\widetilde{\Lambda} / T^{2}$ is indeed a section of the $S^{1}$-bundle $p: \pi^{-1}(\Lambda) / T^{2} \longrightarrow \Lambda$. From the equation $\partial \theta=\pi^{*} \eta$, it follows that $\widetilde{\Theta}$ vanishes on $\widetilde{\Lambda}$. So $\widetilde{\Lambda} / T^{2}$ is indeed a horizontal section.

\subsection{Sufficient condition}

In this subsection, we investigate the inverse question to Proposition 5.4. Namely, given a Lie groupoid $X_{1} \rightrightarrows X_{0}$ and a 3 -cocycle $\eta+\omega \in Z_{D R}^{3}\left(X_{\bullet}\right)$, if $\pi: R_{1} \rightarrow X_{1}$ is an $S^{1}$-bundle and $\theta \in \Omega^{1}\left(R_{1}\right)$ is a connection 1-form of the bundle so that $d \theta=-\pi^{*} \omega$ and the corresponding $S^{1}$-flat bundle $p: \pi^{-1}(\Lambda) / T^{2} \longrightarrow \Lambda$ is holonomy free, does $R_{1}$ always admit a structure of groupoid $S^{1}$-central extension over $X_{1} \rightrightarrows X_{0}$ so that $\theta$ is a pseudo-connection with $\eta+\omega$ being its pseudocurvature? Throughout this subsection, we will keep this assumption and all the notations. Our method is a modification of the one used in [49], where a special case was investigated.

Let $\Lambda_{1}$ be a horizontal section of the flat bundle $p: \pi^{-1}(\Lambda) / T^{2} \longrightarrow \Lambda$. Set $\widetilde{\Lambda}=\tau^{-1}\left(\Lambda_{1}\right) \subset$ $\pi^{-1}(\Lambda)$, which is clearly a $T^{2}$-invariant submanifold. It is also clear that $\operatorname{dim} \widetilde{\Lambda}=\operatorname{dim} \Lambda+2=$ $\operatorname{dim} X_{2}+2$, and $\widetilde{\Theta}$ vanishes when being restricted to $\widetilde{\Lambda}$.

Lemma 5.5 (i). $\pi(\widetilde{\Lambda})=\Lambda$; and

(ii). $\widetilde{\Lambda}$ is a graph over $R_{2}$ 
Proof. (i) is obvious.

(ii) Let $\widetilde{p r}_{12}: R_{1} \times R_{1} \times R_{1} \rightarrow R_{1} \times R_{1}$ be the projection to its first two components. Clearly $\widetilde{p r}_{12}(\widetilde{\Lambda}) \subseteq R_{2}$. Let $(\widetilde{x}, \widetilde{y}) \in R_{2}$ be any point, and write $(x, y) \stackrel{\text { def }}{=} \pi(\widetilde{x}, \widetilde{y})$. Then $(x, y, x y) \in \Lambda$. Assume that $\left(\widetilde{x}_{1}, \widetilde{y}_{1}, \widetilde{z}_{1}\right) \in \widetilde{\Lambda}$ such that $\pi\left(\widetilde{x}_{1}, \widetilde{y}_{1}, \widetilde{z}_{1}\right)=(x, y, x y)$. Then $\widetilde{x}=s \cdot \widetilde{x}_{1}$ and $\widetilde{y}=t \cdot \widetilde{y}_{1}$ for some $s, t \in S^{1}$. Since $\widetilde{\Lambda}$ is $T^{2}$-invariant, it thus follows that $\left(\widetilde{x}, \widetilde{y}, s t \cdot \widetilde{z}_{1}\right)=(s, t) \cdot\left(\widetilde{x}_{1}, \widetilde{y}_{1}, \widetilde{z}_{1}\right) \in \widetilde{\Lambda}$. This shows that $\widetilde{p r}_{12}(\widetilde{\Lambda})=R_{2}$.

To show that $\widetilde{\Lambda}$ is indeed a graph over $R_{2}$, assume that $(\widetilde{x}, \widetilde{y}, \widetilde{z}),\left(\widetilde{x}, \widetilde{y}, \widetilde{z}_{1}\right)$ are two points in $\widetilde{\Lambda}$. Then it is clear that $\pi(\widetilde{x}, \widetilde{y}, \widetilde{z})=\pi\left(\widetilde{x}, \widetilde{y}, \widetilde{z}_{1}\right)$, i.e. $p_{\circ} \tau(\widetilde{x}, \widetilde{y}, \widetilde{z})=p \circ \tau\left(\widetilde{x}, \widetilde{y}, \widetilde{z}_{1}\right)$. Since $\tau(\widetilde{x}, \widetilde{y}, \widetilde{z})$ and $\tau\left(\widetilde{x}, \widetilde{y}, \widetilde{z}_{1}\right) \in \Lambda_{1}$ and $\Lambda_{1}$ is a section for $p$, it follows that $\tau(\widetilde{x}, \widetilde{y}, \widetilde{z})=\tau\left(\widetilde{x}, \widetilde{y}, \widetilde{z}_{1}\right)$. Hence $(\widetilde{x}, \widetilde{y}, \widetilde{z})=(s, t) \cdot\left(\widetilde{x}, \widetilde{y}, \widetilde{z}_{1}\right)$ for some $(s, t) \in T^{2}$, which implies that $s=t=1$ and $\widetilde{z}=\widetilde{z}_{1}$.

Now $\widetilde{\Lambda}$ defines a smooth map $\widetilde{m}^{\prime}: R_{2} \longrightarrow R_{1},(\widetilde{x}, \widetilde{y}) \rightarrow \widetilde{x} * \widetilde{y}$. By construction, the operation $*$ satisfies the condition

$$
(s \cdot \widetilde{x}) *(t \cdot \widetilde{y})=s t \cdot(\widetilde{x} * \widetilde{y})
$$

for all $s, t \in S^{1}$ and $(\widetilde{x}, \widetilde{y}) \in R_{2}$.

Obviously, $\widetilde{m}^{\prime}$ commutes with the projection $\pi$. Therefore for any triple $(\widetilde{x}, \widetilde{y}, \widetilde{z}) \in R_{3}$, both elements $(\widetilde{x} * \widetilde{y}) * \widetilde{z}$ and $\widetilde{x} *(\widetilde{y} * \widetilde{z}) \in R_{1}$ have the same image under the projection $\pi$, so they must differ by a unique element in $S^{1}$. Hence, we obtain a function $g: R_{3} \rightarrow S^{1}$. Note that Eq. (30) implies that $g$ descends to a function on $X_{3}$. Hence, symbolically, we may write

$$
g(x, y, z)=\frac{(\widetilde{x} * \widetilde{y}) * \widetilde{z}}{\widetilde{x} *(\widetilde{y} * \widetilde{z})}, \quad \forall(x, y, z) \in X_{3},
$$

where $(\widetilde{x}, \widetilde{y}, \widetilde{z}) \in R_{3}$ is any point such that $\pi(\widetilde{x}, \widetilde{y}, \widetilde{z})=(x, y, z)$. We call $g(x, y, z)$ the modular function of $\theta$.

Note that $g(x, y, z)$ is independent of the choice of the horizontal section $\Lambda_{1}$ of the flat bundle $p: \pi^{-1}(\Lambda) / T^{2} \longrightarrow \Lambda$, and therefore depends solely on $\theta$.

Proposition 5.6 If the modular function $g(x, y, z)$ is equal to $1, \widetilde{\Lambda}$ defines a Lie groupoid structure on $R_{1}$, which is an $S^{1}$-central extension of $X_{1} \rightrightarrows X_{0}$ with $\theta$ being a pseudo-connection and $\eta+\omega$ the corresponding pseudo-curvature.

Proof. By assumption, we know that $\widetilde{x} * \widetilde{y}$ is indeed associative.

Now we need to show the existence of units. For this purpose, we show that there exists a unique section for the principal $S^{1}$-bundle $R_{1} \stackrel{\pi}{\rightarrow} X_{1}$ over the unit space $\epsilon\left(X_{0}\right)$, namely $\epsilon^{\prime}: \epsilon\left(X_{0}\right) \longrightarrow R_{1}$, $\epsilon(u) \stackrel{\epsilon^{\prime}}{\rightarrow} \widetilde{u}$ such that $(\widetilde{u}, \widetilde{u}, \widetilde{u}) \in \widetilde{\Lambda}$ for any $u \in X_{0}$. Let $\left(\widetilde{u}_{1}, \widetilde{u}_{2}, \widetilde{u}_{3}\right) \in \widetilde{\Lambda}$ be any point such that $\pi\left(\widetilde{u}_{1}, \widetilde{u}_{2}, \widetilde{u}_{3}\right)=(u, u, u)$. Then $\widetilde{u}_{2}=s \cdot \widetilde{u}_{1}$ and $\widetilde{u}_{3}=t \cdot \widetilde{u}_{1}$ for some $s, t \in S^{1}$. Let $\widetilde{u}=\left(s t^{-1}\right) \cdot \widetilde{u}_{1}$. Then

$$
(\widetilde{u}, \widetilde{u}, \widetilde{u})=\left(s t^{-1}, t^{-1}\right) \cdot\left(\widetilde{u}_{1}, \widetilde{u}_{2}, \widetilde{u}_{3}\right) \in \widetilde{\Lambda} .
$$

Assume that $(\widetilde{v}, \widetilde{v}, \widetilde{v})$ is another point in $\widetilde{\Lambda}$ such that $\pi(\widetilde{v}, \widetilde{v}, \widetilde{v})=(u, u, u)$. From the equation $(p \circ \tau)(\widetilde{u}, \widetilde{u}, \widetilde{u})=(p \circ \tau)(\widetilde{v}, \widetilde{v}, \widetilde{v})$, we deduce that $\tau(\widetilde{u}, \widetilde{u}, \widetilde{u})=\tau(\widetilde{v}, \widetilde{v}, \widetilde{v})$. Therefore $(\widetilde{u}, \widetilde{u}, \widetilde{u})=(s, t)$. $(\widetilde{v}, \widetilde{v}, \widetilde{v})$ for some $s, t \in S^{1}$, which means that $\widetilde{u}=s \cdot \widetilde{v}, \widetilde{u}=t \cdot \widetilde{v}, \widetilde{u}=s t \cdot \widetilde{v}$. This implies that $s=t=1$ and hence $\widetilde{u}=\widetilde{v}$.

Next we prove that $\widetilde{u} * \widetilde{x}=\widetilde{x}$ and $\widetilde{x} * \widetilde{v}=\widetilde{x}$ if $\widetilde{s}(\widetilde{x})=u$ and $\widetilde{t}(\widetilde{x})=v$. By construction, we have $\widetilde{u} * \widetilde{u}=\widetilde{u}$. From the associativity assumption, we have

$$
\widetilde{u} *(\widetilde{u} * \widetilde{y})=(\widetilde{u} * \widetilde{u}) * \widetilde{y}=\widetilde{u} * \widetilde{y} \text {, if } \widetilde{s}(\widetilde{y})=u .
$$


We must prove that $\widetilde{x}$ is of the form $\widetilde{u} * \widetilde{y}$. Let $x=\pi(\widetilde{x})$. Since $(u, x, x) \in \Lambda$, there exists $(a, b, c) \in \widetilde{\Lambda}$ such that $\pi(a, b, c)=(u, x, x)$. Thus $\widetilde{u}=s \cdot a$ and $\widetilde{x}=t \cdot c$ for some $s, t \in S^{1}$. So

$$
\left(\widetilde{u}, t s^{-1} \cdot b, \widetilde{x}\right)=\left(s \cdot a, t s^{-1} \cdot b, t \cdot c\right)=\left(s, t s^{-1}\right) \cdot(a, b, c) \in \widetilde{\Lambda}
$$

Thus $\widetilde{x}=\widetilde{u} *\left(t s^{-1} \cdot b\right)$. In conclusion, we have $\widetilde{u} * \widetilde{x}=\widetilde{x}$. Similarly, one proves that $\widetilde{x} * \widetilde{v}=\widetilde{x}$.

Finally, we need to show the existence of inverse. For any $\widetilde{x} \in R_{1}$, let $x=\pi(\widetilde{x})$ and $\widetilde{s}(\widetilde{x})=v$. Since $\left(x, x^{-1}, v\right) \in \Lambda$, there exists $\left(\widetilde{x}_{1}, \widetilde{y}_{1}, \widetilde{z}_{1}\right) \in \widetilde{\Lambda}$ such that $\pi\left(\widetilde{x}_{1}, \widetilde{y}_{1}, \widetilde{z}_{1}\right)=\left(x, x^{-1}, v\right)$. One may assume that $\widetilde{x}_{1}=\widetilde{x}$ by using the $T^{2}$-action. Since $\pi \widetilde{z}_{1}=\pi \widetilde{v}$, we have $\widetilde{v}=t \cdot \widetilde{z}_{1}$. Thus $\left(\widetilde{x}, t \cdot \widetilde{y}_{1}, \widetilde{v}\right)=(1, t) \cdot\left(\widetilde{x}, \widetilde{y}_{1}, \widetilde{z}_{1}\right) \in \widetilde{\Lambda}$. This shows that the right inverse of $\widetilde{x}$ exists. Similarly, one shows that the left inverse exists as well. It is then standard that the left and right inverses must coincide. This concludes the proof.

In general, the modular function is not necessarily equal to 1 . Nevertheless, we have the following characterization.

Proposition 5.7 The modular function $g: X_{3} \rightarrow S^{1}$ defines an $\mathbb{R} / \mathbb{Z}$-valued groupoid 3-cocycle. I.e., $d g=0, \quad \partial g=1$.

Proof. Let $(x(t), y(t), z(t))$ be any smooth curve in $X_{3}$, and $(\widetilde{x}(t), \widetilde{y}(t), \widetilde{z}(t))$ a smooth curve in $R_{3}$ such that $\pi(\widetilde{x}(t), \widetilde{y}(t), \widetilde{z}(t))=(x(t), y(t), z(t))$. Write $\widetilde{u}(t)=\widetilde{x}(t) * \widetilde{y}(t), \widetilde{v}(t)=\widetilde{y}(t) * \widetilde{z}(t)$, and $\widetilde{h_{1}}(t)=(\widetilde{x}(t) * \widetilde{y}(t)) * \widetilde{z}(t), \widetilde{h_{2}}(t)=\widetilde{x}(t) *(\widetilde{y}(t) * \widetilde{z}(t))$.

Since $(\widetilde{x}(t), \widetilde{y}(t), \widetilde{u}(t)),\left(\widetilde{u}(t), \widetilde{z}(t), \widetilde{h_{1}}(t)\right) \in \widetilde{\Lambda}$, we have

$$
\begin{aligned}
& \dot{\widetilde{x}}(t)\lrcorner \theta+\dot{\widetilde{y}}(t)\lrcorner \theta-\dot{\widetilde{u}}(t)\lrcorner \theta=\eta(\dot{\widetilde{x}}(t) * \dot{\widetilde{y}}(t)), \text { and } \\
& \left.\dot{\widetilde{u}}(t)\lrcorner \theta+\dot{\widetilde{z}}(t)\lrcorner \theta-\dot{\widetilde{h}}_{1}(t)\right\lrcorner \theta=\eta(\dot{\widetilde{u}}(t) * \dot{\widetilde{z}}(t)),
\end{aligned}
$$

where, by abuse of notation, we use the same symbol $*$ to denote the induced tangent map $T R_{2} \rightarrow$ $T R_{1}$. It follows that

$$
\left.\left.\dot{\widetilde{h}}_{1}(t) \downarrow \theta=\dot{\widetilde{x}}(t)\right\lrcorner \theta+\dot{\widetilde{y}}(t) \downarrow \theta+\dot{\widetilde{z}}(t)\right\lrcorner \theta-\eta(\dot{\widetilde{x}}(t) * \dot{\widetilde{y}}(t))-\eta(\dot{\widetilde{u}}(t) * \dot{\widetilde{z}}(t))
$$

Similarly, one proves that

$$
\left.\left.\left.\left.\dot{\widetilde{h}}_{2}(t)\right\lrcorner \theta=\dot{\widetilde{x}}(t)\right\lrcorner \theta+\dot{\widetilde{y}}(t)\right\lrcorner \theta+\dot{\widetilde{z}}(t)\right\lrcorner \theta-\eta(\dot{\widetilde{x}}(t) * \dot{\widetilde{v}}(t))-\eta(\dot{\widetilde{y}}(t) * \dot{\widetilde{z}}(t))
$$

Since $\partial \eta=0, \operatorname{Eqs}(31)$ and $(32)$ imply that $\left.\left.\dot{\widetilde{h}}_{1}(t)\right\lrcorner \theta=\dot{\widetilde{h}}_{2}(t)\right\lrcorner \theta$.

Let $f(t)=\frac{\widetilde{h_{1}}(t)}{h_{2}(t)}$. Therefore $\widetilde{h_{1}}(t)=f(t) \cdot \widetilde{h_{2}}(t)$, which implies that

$$
\dot{\widetilde{h}}_{1}(t)=f(t) \cdot \dot{\widetilde{h}}_{2}(t)+\dot{f}(t) \xi
$$

where $\xi$ is the Euler vector field on $R_{1}$. Pairing with $\theta$ on both sides, one obtains that $\dot{f}(t)=0$.

Finally, the identity $\partial g=1$ can be verified directly.

Corollary 5.8 (i). For any $y \in X_{1}$, we have $g(s(y), y, t(y))=1$. In particular, $\forall u \in X_{0}$, we have $g(u, u, u)=1$; 
(ii). If $X_{1}$ is s-connected, then $g(x, y, z)=1$.

Proof. Since $\partial g=1$, we have

$$
g(y, z, w) g(x y, z, w)^{-1} g(x, y z, w) g(x, y, z w)^{-1} g(x, y, z)=1 .
$$

By letting $x=s(y)$ and $z=t(y)$, we obtain that $g(s(y), y, t(y))=1$.

For any $(x, y, z) \in X_{3}$, if $X_{1}$ is $s$-connected, then $x$ can be connected to $s(y)$ by a smooth path in the $t$-fiber $t^{-1}(s(y))$ while $z$ can be connected to $t(y)$ by a smooth path in the $s$-fiber $s^{-1}(t(y))$. In other words, $(x, y, z)$ and $(s(y), y, t(y))$ belong to the same connected component of $X_{3}$. Thus $g(x, y, z)=1$ according to Proposition 5.7.

An immediate consequence is the following

Proposition 5.9 Let $X_{1} \rightrightarrows X_{0}$ be an s-connected Lie groupoid, and $\eta+\omega \in Z_{D R}^{3}\left(X_{\bullet}\right)$ a 3cocycle, where $\eta \in \Omega^{1}\left(X_{2}\right)$ and $\omega \in \Omega^{2}\left(X_{1}\right)$. Assume that $\omega$ represents an integer cohomology class in $H_{D R}^{2}\left(X_{1}\right)$, so that there exists an $S^{1}$-bundle $\pi: R_{1} \rightarrow X_{1}$ with a connection 1-form $\theta \in \Omega^{1}\left(R_{1}\right)$, whose curvature is $-\omega$. If the associated $S^{1}$-bundle $p: \pi^{-1}(\Lambda) / T^{2} \longrightarrow \Lambda$ as in diagram (20) is holonomy free, then $R_{1} \rightarrow X_{1}$ is a Lie groupoid $S^{1}$-central extension with $\theta$ being a pseudo-connection and $\eta+\omega$ being the pseudo-curvature. In particular, $\eta+\omega$ is of integer class in $H^{3}\left(X_{\bullet}, \mathbb{Z}\right)$.

\subsection{Properties of 3-cocycles}

In this subsection we study some geometric properties of 3-cocycles of the De-Rham double complex of a Lie groupoid, which are important for our constructions in the next section.

Let $\eta+\omega \in Z_{D R}^{3}\left(X_{\bullet}\right)$ be a de-Rham three-cocycle, where $\eta \in \Omega^{1}\left(X_{2}\right)$ and $\omega \in \Omega^{2}\left(X_{1}\right)$. Then

$$
\partial \eta=0, \quad \partial \omega+d \eta=0, \quad d \omega=0 .
$$

By $X_{1}^{s}$ and $X_{1}^{t}$ we denote the $s$ - and $t$-fibrations of $X_{1} \rightrightarrows X_{0}$, respectively. Define a leafwise one-form $\lambda^{r}$ on $X_{1}^{t}$ by

$$
\lambda^{r}\left(\delta_{x}\right)=\eta\left(r_{x^{-1} *} \delta_{x}, 0_{x}\right), \quad \forall \delta_{x} \in T_{x} X_{1}^{t} .
$$

Similarly, let $\lambda^{l}$ be the leafwise one-form on $X_{1}^{s}$ given by

$$
\lambda^{l}\left(\delta_{x}\right)=\eta\left(0_{x}, l_{x^{-1} *} \delta_{x}\right), \quad \forall \delta_{x} \in T_{x} X_{1}^{s} .
$$

Here $r_{x^{-1}}$ and $l_{x^{-1}}$ denote the right and the left translations, respectively.

Note that $\lambda^{r}$ (or $\lambda^{l}$ ) is in general not right (left)-invariant.

By $A \rightarrow X_{0}$ we denote the Lie algebroid of $X_{1} \rightrightarrows X_{0}$. For any section $V \in \Gamma(A)$, we denote, respectively, by $\vec{V}$ and $\overleftarrow{V}$ the right invariant and the left invariant vector fields on $X_{1}$ corresponding to $V$.

Lemma 5.10 For any $V \in \Gamma(A)$,

(i). $\eta\left(\vec{V}(x), 0_{y}\right)=\lambda^{r}(\vec{V}(x y))-\lambda^{r}(\vec{V}(x)), \forall(x, y) \in X_{2}$;

(ii). $\eta\left(0_{x}, \overleftarrow{V}(y)\right)=\lambda^{l}(\overleftarrow{V}(x y))-\lambda^{l}(\overleftarrow{V}(y)), \forall(x, y) \in X_{2}$ 
(iii). $\lambda^{r}(\vec{V})(u)=\lambda^{l}(\overleftarrow{V})(u)=0, \forall u \in X_{0} ;$ and

(iv). $\eta\left(\vec{V}(x),-\overleftarrow{V}\left(x^{-1}\right)\right)=\lambda^{l}(\overleftarrow{V})\left(x^{-1}\right)-\lambda^{r}(\vec{V})(x), \forall x \in X_{1}$

Proof. Consider the curve $r(t)=(\exp t \vec{V}, x, y)$ in $X_{3}$ through the point $(s(x), x, y)$. By definition, we have

$$
\dot{r}(0)\lrcorner \partial \eta=\eta\left(0_{x}, 0_{y}\right)-\eta\left(\vec{V}(x), 0_{y}\right)+\eta\left(\vec{V}(s(x)), 0_{x y}\right)-\eta\left(\vec{V}(s(x)), 0_{x}\right) .
$$

Thus (i) follows immediately since $\partial \eta=0$. Similarly (ii) can be proved by considering the curve $(x, y, \exp t \overleftarrow{V})$ through the point $(x, y, t(y))$. (iii) follows from (i) and (ii) by taking $x=y=u \in X_{0}$. Finally, using (i)-(iii), we have

$$
\begin{aligned}
\eta\left(\vec{V}(x),-\overleftarrow{V}\left(x^{-1}\right)\right) & =\eta\left(\vec{V}(x), 0_{x^{-1}}\right)-\eta\left(0_{x}, \overleftarrow{V}\left(x^{-1}\right)\right) \\
& =\left[\lambda^{r}(\vec{V})\left(x x^{-1}\right)-\lambda^{r}(\vec{V})(x)\right]-\left[\lambda^{l}(\overleftarrow{V})\left(x x^{-1}\right)-\lambda^{l}(\overleftarrow{V})\left(x^{-1}\right)\right] \\
& =\lambda^{l}(\overleftarrow{V})\left(x^{-1}\right)-\lambda^{r}(\vec{V})(x)
\end{aligned}
$$

Thus (iv) follows.

For any $V \in \Gamma(A)$, by $V^{r}$ and $V^{l}$ we denote the vector fields on $X_{2}$ given by $V^{r}(x, y)=$ $\left(\vec{V}(x), 0_{y}\right)$ and $V^{l}(x, y)=\left(0_{x}, \overleftarrow{V}(y)\right), \forall(x, y) \in X_{2}$. It is clear that the flows of $V^{r}$ and $V^{l}$ are respectively, given by

$$
\varphi_{t}(x, y)=(\exp (t \vec{V}) x, y), \quad \psi_{t}(x, y)=(x, y \exp (t \overleftarrow{V}))
$$

Lemma 5.11 For any $V, W \in \Gamma(A)$,

(i). $(d \eta)\left(V^{r}, W^{l}\right)(x, y)=\vec{V}\left(\lambda^{l}(\overleftarrow{W})\right)(x y)-\overleftarrow{W}\left(\lambda^{r}(\vec{V})\right)(x y)$

(ii). $(d \eta)\left(V^{r}, W^{r}\right)(x, y)=\left(d \lambda^{r}\right)(\vec{V}, \vec{W})(x y)-\left(d \lambda^{r}\right)(\vec{V}, \vec{W})(x)$.

(iii). $(d \eta)\left(V^{l}, W^{l}\right)(x, y)=\left(d \lambda^{l}\right)(\overleftarrow{V}, \overleftarrow{W})(x y)-\left(d \lambda^{l}\right)(\overleftarrow{V}, \overleftarrow{W})(y)$

Proof. (i) From Eq. (34), one easily sees that the vector fields $V^{r}$ and $W^{l}$ commute with each other: $\left[V^{r}, W^{l}\right]=0$.

According to Lemma 5.10 (ii), $\eta\left(W^{l}\right)(x, y)=\lambda^{l}(\overleftarrow{W}(x y))-\lambda^{l}(\overleftarrow{W}(y))$. It thus follows that

$$
V^{r}\left(\eta\left(W^{l}\right)\right)(x, y)=\left.\frac{d}{d t}\right|_{t=0}\left[\lambda^{l}\left(\overleftarrow{W}(\exp (t \vec{V}) x y)-\lambda^{l}(\overleftarrow{W}(y))\right]=\vec{V}\left(\lambda^{l}(\overleftarrow{W})\right)(x y)\right.
$$

Similarly, one shows that $W^{l}\left(\eta\left(V^{r}\right)\right)(x, y)=\overleftarrow{W}\left(\lambda^{r}(\vec{V})(x y)\right.$. (i) thus follows.

(ii) We have

$$
\begin{aligned}
V^{r}\left(\eta\left(W^{r}\right)\right)(x, y) & =V^{r}\left[\lambda^{r}(\vec{W}(x y))-\lambda^{r}(\vec{W}(x))\right] \\
& =\left.\frac{d}{d t}\right|_{t=0}\left[\lambda^{r}(\vec{W}(\exp (t \vec{V}) x y))-\lambda^{r}(\vec{W}(\exp (t \vec{V}) x))\right] \\
& =\vec{V}\left(\lambda^{r}(\vec{W})\right)(x y)-\vec{V}\left(\lambda^{r}(\vec{W})\right)(x) .
\end{aligned}
$$


Hence

$$
\begin{aligned}
(d \eta)\left(V^{r}, W^{r}\right)(x, y) & =V^{r}\left(\eta\left(W^{r}\right)\right)(x, y)-W^{r}\left(\eta\left(V^{r}\right)\right)(x, y)-\eta\left(\left[V^{r}, W^{r}\right]\right)(x, y) \\
& =\left(d \lambda^{r}\right)(\vec{V}, \vec{W})(x y)-\left(d \lambda^{r}\right)(\vec{V}, \vec{W})(x) .
\end{aligned}
$$

(iii) can be proved similarly.

Proposition 5.12 Assume that $\eta+\omega \in Z_{D R}^{3}\left(X_{\bullet}\right)$ is a 3-cocycle.

(i). $\epsilon^{*} \omega=-d \eta_{0}$;

(ii). For any $V, W \in \Gamma(A), \omega(\vec{V}, \overleftarrow{W})=\vec{V} \lambda^{l}(\overleftarrow{W})-\overleftarrow{W} \lambda^{r}(\vec{V})$

(iii). $\omega-d \lambda^{r} \in \Omega^{2}\left(X^{t}\right)$ is a right invariant (leafwise) closed 2-form, and therefore induces a Lie algebroid 2-cocycle $\omega^{r} \in \Gamma\left(\wedge^{2} A^{*}\right)$.

(iv). $\omega-d \lambda^{l} \in \Omega^{2}\left(X^{s}\right)$ is a left invariant (leafwise) closed 2-form, and therefore induces a Lie algebroid 2-cocycle $\omega^{l} \in \Gamma\left(\wedge^{2} A^{*}\right)$.

(v). $\omega^{r}$ and $\omega^{l}$ are related by

$$
\omega^{r}+\omega^{l}+\rho^{*} d \eta_{0}=0
$$

i.e. $\omega^{r}$ and $-\omega^{l}$ are cohomologous Lie algebroid 2-cocycles. Here $\rho: A \rightarrow T X_{0}$ is the anchor of the Lie algebroid A.

Proof. It is not difficult to see that $m_{*} V^{r}(x, y)=\vec{V}(x y)$ and $m_{*} W^{l}(x, y)=\overleftarrow{W}(x y)$. Thus

$$
(\partial \omega)\left(V^{r}, W^{l}\right)(x, y)=-\omega(\vec{V}, \overleftarrow{W})(x y)
$$

On the other hand, according to Lemma 5.11(i), we have

$$
(d \eta)\left(V^{r}, W^{l}\right)(x, y)=\vec{V}\left(\lambda^{l}(\overleftarrow{W})\right)(x y)-\overleftarrow{W}\left(\lambda^{r}(\vec{V})\right)(x y)
$$

Since $\partial \omega+d \eta=0$, it thus follows that

$$
\omega(\vec{V}, \overleftarrow{W})(x y)=\vec{V} \lambda^{l}(\overleftarrow{W})(x y)-\overleftarrow{W} \lambda^{r}(\vec{V})(x y)
$$

(ii) thus follows by letting $y=t(x)$.

For (iii), we note that

$$
(\partial \omega)\left(V^{r}, W^{r}\right)(x, y)=\omega(\vec{V}, \vec{W})(x)-\omega(\vec{V}, \vec{W})(x y) .
$$

The conclusion thus follows from Lemma 5.11. (iv) can be proved similarly.

Finally, consider the map $\kappa: X_{1} \rightarrow X_{2}$ as in Eq. (28). It is clear that

$$
\kappa_{*} \vec{V}(x)=\left(\vec{V}(x),-\overleftarrow{V}\left(x^{-1}\right)\right)
$$

Thus

$$
\begin{aligned}
\left(\kappa^{*} \eta\right)(\vec{V})(x) & =\eta\left(\vec{V}(x),-\overleftarrow{V}\left(x^{-1}\right)\right) \quad(\text { by Lemma } 5.10(\mathrm{iv})) \\
& =-\lambda^{r}(\vec{V})(x)+\lambda^{l}(\overleftarrow{V})\left(x^{-1}\right) \\
& =\left(-\lambda^{r}-\iota^{*} \lambda^{l}\right)(\vec{V})(x)
\end{aligned}
$$


It follows that $\kappa^{*} \eta=-\lambda^{r}-\iota^{*} \lambda^{l}$. Here $\kappa^{*} \eta$ is considered as a fiberwise one-form on $X_{1}^{t}$ by restriction.

For any $V \in \Gamma(A)$, write $\bar{V}\left(x, x^{-1}\right)=\left(\vec{V}(x),-\overleftarrow{V}\left(x^{-1}\right)\right) \in T_{\left(x, x^{-1}\right)} X_{2}$. Eq. (35) means that $\kappa_{*} \vec{V}(x)=\bar{V}\left(x, x^{-1}\right)$. Hence for any $V, W \in \Gamma(A)$,

$$
\begin{aligned}
(d \eta)\left(\bar{V}\left(x, x^{-1}\right), \bar{W}\left(x, x^{-1}\right)\right) & =d\left(\kappa^{*} \eta\right)(\vec{V}(x), \vec{W}(x)) \\
& =-\left(d \lambda^{r}\right)(\vec{V}(x), \vec{W}(x))-\left(d \lambda^{l}\right)\left(\iota_{*} \vec{V}(x), \iota_{*} \vec{W}(x)\right) \\
& =-\left(d \lambda^{r}\right)(\vec{V}(x), \vec{W}(x))-\left(d \lambda^{l}\right)\left(\overleftarrow{V}\left(x^{-1}\right), \overleftarrow{W}\left(x^{-1}\right)\right) .
\end{aligned}
$$

On the other hand, we have $m_{*} \bar{V}\left(x, x^{-1}\right)=\epsilon_{*} s_{*} \vec{V}(x)$ and $m_{*} \bar{W}\left(x, x^{-1}\right)=\epsilon_{*} s_{*} \vec{W}(x)$. To see this, note that $\left(\exp t \vec{V} \cdot x,(\exp t \vec{V} \cdot x)^{-1}\right)$ is the flow generated by $\bar{V}\left(x, x^{-1}\right)$ on $X_{2}$. Thus we have

$$
(\partial \omega)\left(\bar{V}\left(x, x^{-1}\right), \bar{W}\left(x, x^{-1}\right)\right)=\omega(\vec{V}, \vec{W})(x)+\omega(\overleftarrow{V}, \overleftarrow{W})\left(x^{-1}\right)-\omega\left(s_{*} \vec{V}(x), s_{*} \vec{W}(x)\right)
$$

(v) thus follows immediately.

\subsection{Lie algebroid central extensions}

As in the last subsection, let $\eta+\omega \in Z_{D R}^{3}\left(X_{\bullet}\right)$ be a de-Rham 3-cocycle of a Lie groupoid $X_{1} \rightrightarrows X_{0}$ and $\omega$ represents an integer cohomology class in $H_{D R}^{2}\left(X_{1}\right)$. Let $\pi: R_{1} \rightarrow X_{1}$ be an $S^{1}$-bundle and $\theta \in \Omega^{1}\left(R_{1}\right)$ a connection 1 -form of the bundle so that $d \theta=-\pi^{*} \omega$.

Recall that, for a given Lie algebroid $A \rightarrow M$, any Lie algebroid 2-cocycle $\gamma \in \Gamma\left(\wedge^{2} A^{*}\right)$ induces a Lie algebroid central extension $\widetilde{A}=A \oplus(M \times \mathbb{R})$ as follows. The anchor map $\widetilde{\rho}(V+f)=\rho(V)$, $\forall V \in \Gamma(A)$ and $f \in C^{\infty}(M)$, and the bracket is

$$
[V+f, W+g]=[V, W]+(\rho(V)(g)-\rho(W)(f)+\gamma(V, W)),
$$

$\forall V, W \in \Gamma(A)$ and $f, g \in C^{\infty}(M)$. Denote by $\widetilde{A}^{r}$ and $\widetilde{A}^{l}$ the Lie algebroid central extensions of $A$ by the 2 -cocycles $\omega^{r}$ and $-\omega^{l}$, respectively. Then $\widetilde{A}^{r}$ and $\widetilde{A}^{l}$ are isomorphic, while the isomorphism is given by

$$
\left.\widetilde{A}^{r} \longrightarrow \widetilde{A}^{l}, \quad V+f \rightarrow V+(f+\rho(V)\lrcorner \eta_{0}\right), \quad \forall V \in \Gamma(A) .
$$

Let $\theta^{r}=\theta+\pi^{*} \lambda^{r}$ and $\theta^{l}=\theta+\pi^{*} \lambda^{l}$ be the fiberwise one-forms on $R_{1}^{\widetilde{t}}$ and $R_{1}^{\widetilde{s}}$, respectively. Then $\theta^{r}$ is a fiberwise connection one-form on the fiberwise $S^{1}$-principal bundle $R_{1}^{\widetilde{t}} \rightarrow X_{1}^{t}$ with curvature being $-\omega+d \lambda^{r}$, while $\theta^{l}$ is a fiberwise connection one-form on the fiberwise $S^{1}$-principal bundle $R_{1}^{\widetilde{s}} \rightarrow X_{1}^{s}$ with curvature being $-\omega+d \lambda^{l}$. For any $V \in \Gamma(A)$, denote by $\widehat{\vec{V}} \in \mathfrak{X}\left(R_{1}^{\widetilde{\tau}}\right)$ the horizontal lift of $\vec{V}$ with respect to $\theta^{r}$, and $\overleftarrow{V} \in \mathfrak{X}\left(R_{1}^{\widetilde{s}}\right)$ the horizontal lift of $\overleftarrow{V}$ with respect to $\theta^{l}$. I.e.,

$$
\left\{\begin{array}{l}
\widehat{\vec{V}}\lrcorner\left(\theta+\pi^{*} \lambda^{r}\right)=0, \\
\pi_{*} \overrightarrow{\widehat{V}}=\vec{V}
\end{array}\right.
$$

and

$$
\left\{\begin{array}{l}
\widehat{\overleftarrow{V}}\lrcorner\left(\theta+\pi^{*} \lambda^{l}\right)=0 \\
\pi_{*} \overleftarrow{\overleftarrow{V}}=\overleftarrow{V}
\end{array}\right.
$$


Introduce linear maps $\varphi: \Gamma\left(\widetilde{A}^{r}\right) \longrightarrow \mathfrak{X}\left(R_{1}\right)$ and $\psi: \Gamma\left(\widetilde{A}^{l}\right) \longrightarrow \mathfrak{X}\left(R_{1}\right)$, respectively, by

$$
\varphi: V+f \longrightarrow \widehat{\vec{V}}+\left(\pi^{*} s^{*} f\right) \xi
$$

and

$$
\psi: V+f \longrightarrow \widehat{\overleftarrow{V}}+\left(\pi^{*} t^{*} f\right) \xi
$$

$\forall V \in \Gamma(A)$ and $f \in C^{\infty}\left(X_{0}\right)$. Set

$$
\mathcal{D}_{s}=\varphi \Gamma\left(\widetilde{A}^{r}\right), \text { and } \mathcal{D}_{t}=\psi \Gamma\left(\widetilde{A}^{l}\right) \subset \mathfrak{X}\left(R_{1}\right)
$$

Proposition 5.13 (i). Both $\varphi$ and $\psi$ are Lie algebra homomorphisms.

(ii). Vector fields in $\mathcal{D}_{s}$ and $\mathcal{D}_{t}$ mutually commute.

Proof. $\forall V, W \in \Gamma(A)$ and $f, g \in C^{\infty}\left(X_{0}\right)$, we have

$$
\begin{aligned}
& {\left[\widehat{\vec{V}}+\left(\pi^{*} s^{*} f\right) \xi, \widehat{\vec{W}}+\left(\pi^{*} s^{*} g\right) \xi\right] } \\
= & {[\widehat{\vec{V}}, \widehat{\vec{W}}]+\left[\widehat{\vec{V}},\left(\pi^{*} s^{*} g\right) \xi\right]+\left[\left(\pi^{*} s^{*} f\right) \xi, \widehat{\vec{W}}\right] }
\end{aligned}
$$

Since the vector field $\widehat{\vec{V}}$ is $S^{1}$-invariant, we have $[\widehat{\vec{V}}, \xi]=0$. On the other hand, since $s_{*} \pi_{*} \widehat{\vec{V}}=$ $s_{*} \vec{V}=\rho(V)$, it follows that $\widehat{\vec{V}}\left(\pi^{*} s^{*} g\right)=\rho(V) g$. Therefore,

$$
\left[\widehat{\vec{V}},\left(\pi^{*} s^{*} g\right) \xi\right]=\left(\pi^{*} s^{*} g\right)[\widehat{\vec{V}}, \xi]+\left(\widehat{\vec{V}}\left(\pi^{*} s^{*} g\right)\right) \xi=(\rho(V) g) \xi
$$

Similarly, one proves that $\left[\left(\pi^{*} s^{*} f\right) \xi, \widehat{\vec{W}}\right]=-(\rho(W) f) \xi$. Finally note that

$$
\widehat{[\vec{V}, \widehat{\vec{W}}]}=\widehat{[V, W]}+\pi^{*} s^{*} \omega^{r}(V, W) \xi
$$

Hence it follows that $\varphi$ is indeed a Lie algebra homomorphism. Similarly, one proves that $\psi$ is also a Lie algebra homomorphism.

For the second part, for any $V, W \in \Gamma(A)$ and $f, g \in C^{\infty}\left(X_{0}\right)$, we have

$$
\begin{aligned}
{[\varphi(V+f), \psi(W+g)] } & =\left[\widehat{\vec{V}}+\left(\pi^{*} s^{*} f\right) \xi, \widehat{\overleftarrow{W}}+\left(\pi^{*} t^{*} g\right) \xi\right] \\
& =[\widehat{\vec{V}}, \widehat{\overleftarrow{W}}]+\left[\widehat{\vec{V}},\left(\pi^{*} t^{*} g\right) \xi\right]+\left[\left(\pi^{*} s^{*} f\right) \xi, \widehat{\overleftarrow{W}}\right]
\end{aligned}
$$

Now $\left[\widehat{\vec{V}},\left(\pi^{*} t^{*} g\right) \xi\right]=\left[\left(\pi^{*} s^{*} f\right) \xi, \widehat{\overleftarrow{W}}\right]=0$ since $t_{*} \pi_{*} \widehat{\vec{V}}=t_{*} \vec{V}=0$ and $s_{*} \pi_{*} \widehat{\overleftarrow{W}}=s_{*} \overleftarrow{W}=0$

It remains to show that $[\widehat{\vec{V}}, \widehat{\overleftarrow{W}}]=0$. For this, first of all, note that $\pi_{*}[\widehat{\vec{V}}, \widehat{\overleftarrow{W}}]=[\vec{V}, \overleftarrow{W}]=0$ By Eqs. (37), (38), we have $\widehat{\vec{V}}\lrcorner \theta=-\pi^{*}\left(\lambda^{r}(\vec{V})\right)$ and $\left.\overleftarrow{W}\right\lrcorner \theta=-\pi^{*}\left(\lambda^{l}(\overleftarrow{W})\right)$. It thus follows that

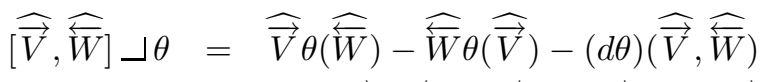

$$
\begin{aligned}
& =\pi^{*}\left[-\vec{V} \lambda^{l}(\overleftarrow{W})+\overleftarrow{W} \lambda^{r}(\vec{V})+\omega(\vec{V}, \overleftarrow{W})\right] \text { (by Proposition } 5.12 \text { (ii)) } \\
& =0 \text {. }
\end{aligned}
$$

This concludes the proof of the proposition. $\square$ 
Introduce distributions $\mathcal{F}_{s}$ and $\mathcal{F}_{t}$ on $\pi^{-1}(\Lambda)$ as follows. For any $(\widetilde{x}, \widetilde{y}, \widetilde{z}) \in \pi^{-1}(\Lambda)$,

$$
\begin{aligned}
\left.\left.\mathcal{F}_{s}\right|_{(\widetilde{x}, \widetilde{y}, \widetilde{z})}=\{(\varphi(V+f)(\widetilde{x}), 0, \varphi(V+f))(\widetilde{z})) \mid \forall V \in \Gamma(A), f \in C^{\infty}\left(X_{0}\right)\right\} \\
\left.\left.\mathcal{F}_{t}\right|_{(\widetilde{x}, \widetilde{y}, \widetilde{z})}=\{(0, \psi(V+f)(\widetilde{y}), \psi(V+f))(\widetilde{z})) \mid \forall V \in \Gamma(A), f \in C^{\infty}\left(X_{0}\right)\right\} .
\end{aligned}
$$

It is clear, from Proposition 5.13, that both $\mathcal{F}_{s}$ and $\mathcal{F}_{t}$ are integrable distributions. By $\mathcal{F}$, we denote the distribution on $\pi^{-1}(\Lambda)$ defined by the equation $\widetilde{\Theta}=0$. According to Eq. $(22), \mathcal{F}$ is an integrable distribution.

Proposition 5.14 We have $\mathcal{F}_{s} \subseteq \mathcal{F}$ and $\mathcal{F}_{t} \subseteq \mathcal{F}$.

Proof. Let $v=(\varphi(V+f)(\widetilde{x}), 0, \varphi(V+f))(\widetilde{z}))$. Then

$$
\begin{aligned}
v\lrcorner(\theta, \theta,-\theta) & =(\widehat{\vec{V}}\lrcorner \theta)(\widetilde{x})+f(s \circ \pi(\widetilde{x}))-(\widehat{\vec{V}}\lrcorner \theta)(\widetilde{z})-f(s \circ \pi(\widetilde{z})) \\
& =-\lambda^{r}(\vec{V})(x)+\lambda^{r}(\vec{V})(z) .
\end{aligned}
$$

On the other hand,

$$
v\lrcorner \pi^{*} \eta=(\vec{V}(x), 0) \downarrow \eta=\eta\left(\vec{V}(x), 0_{y}\right) .
$$

Thus

$$
v\lrcorner \widetilde{\Theta}=v\lrcorner \Theta-v\lrcorner \pi^{*} \eta=0,
$$

according to Lemma 5.10 (i). Hence we have proved that $\mathcal{F}_{s} \subseteq \mathcal{F}$. Similarly, one shows that $\mathcal{F}_{t} \subseteq \mathcal{F}$.

We are now ready to prove the main theorem of this section.

Theorem 5.15 Let $X_{1} \rightrightarrows X_{0}$ be an s-connected Lie groupoid, and $\eta+\omega \in Z_{D R}^{3}\left(X_{\bullet}\right)$ a de-Rham 3-cocycle, where $\eta \in \Omega^{1}\left(X_{2}\right)$ and $\omega \in \Omega^{2}\left(X_{1}\right)$. Assume that $\omega$ represents an integer cohomology class in $H_{D R}^{2}\left(X_{1}\right)$, so that there exists an $S^{1}$-bundle $\pi: R_{1} \rightarrow X_{1}$ with a connection $\theta \in \Omega^{1}\left(R_{1}\right)$, whose curvature is $-\omega$. Assume that $\epsilon^{*} R_{1}$ endowed with the flat connection $\epsilon^{*} \theta-\pi^{*} \epsilon_{2}^{*} \eta$ is holonomy free. (Here $\epsilon: X_{0} \rightarrow X_{1}$ and $\epsilon_{2}: X_{0} \rightarrow X_{2}$ are the respective identity morphisms.) Then $R_{1} \rightrightarrows R_{0}$, where $R_{0}=X_{0}$, admits in a natural way the structure of a Lie groupoid, such that it becomes an $S^{1}$-central extension of $X_{1} \rightrightarrows X_{0}$ and $\eta+\omega$ the pseudo-curvature of $\theta$. In particular, $\eta+\omega$ is of integer class in $H^{3}\left(X_{\bullet}, \mathbb{Z}\right)$.

Proof. Take a horizontal section $\epsilon^{\prime}$ of the bundle $\left.R_{1}\right|_{X_{0}} \rightarrow \epsilon\left(X_{0}\right): \epsilon(u) \rightarrow \widetilde{u}, \forall u \in X_{0}$. Consider the foliation in $R_{1} \times R_{1} \times R_{1}$ defined by $\mathcal{F}_{s}+\mathcal{F}_{t}$. Let

$$
I=\left\{(\widetilde{u}, \widetilde{u}, \widetilde{u}) \mid \forall u \in X_{0}\right\} .
$$

Then $I$ is transversal to the foliation $\mathcal{F}_{s}+\mathcal{F}_{t}$. By the method of characteristics [9], there is a minimal $\mathcal{F}_{s}+\mathcal{F}_{t}$-invariant submanifold $\widetilde{\Lambda}$ containing $I$ which is immersed in $R_{1} \times R_{1} \times R_{1}$. Proposition 5.14 implies that $\widetilde{\Theta}=0$ when being restricted to $\widetilde{\Lambda}$.

It is clear that $\widetilde{\Lambda}$ is $T^{2}$-invariant since the $T^{2}$-generating vector fields $\xi_{1} \in \mathcal{F}_{\alpha}$ and $\xi_{2} \in \mathcal{F}_{\beta}$. Now we need to show that $\widetilde{\Lambda}$ is a graph over $R_{2}$. Let $\operatorname{pr}_{12}: R_{1} \times R_{1} \times R_{1} \rightarrow R_{1} \times R_{1}$ be the natural projection onto the first two coordinates: $\operatorname{pr}_{12}(x, y, z)=(x, y)$. First, we show that $\operatorname{pr}_{12}(\widetilde{\Lambda})=R_{2}$.

Note that $(\widetilde{x}, \widetilde{y}, \widetilde{z}) \in \widetilde{\Lambda}$ if and only if $\widetilde{x}=\varphi^{\alpha} \widetilde{u}, \widetilde{y}=\varphi^{\beta} \widetilde{u}$, and $z=\varphi^{\alpha} \varphi^{\beta} \widetilde{u}$ for some $u \in X_{0}$, where $\varphi^{\alpha}$ is a product of flows in $\mathcal{D}_{s}$ and $\varphi^{\beta}$ is a product of flows in $\mathcal{D}_{t}$. 
Since $\widetilde{t}_{*} \mathcal{D}_{s}=0$ and $\widetilde{s}_{*} \mathcal{D}_{t}=0$, the flow of $\mathcal{D}_{s}$ preserves $\widetilde{t}$-fibres and similarly the flow of $\mathcal{D}_{t}$ preserves $\widetilde{s}$-fibres; thus

$$
\widetilde{t}(\widetilde{x})=\widetilde{t}\left(\varphi^{\alpha} \widetilde{u}\right)=u,
$$

and

$$
\widetilde{s}(\widetilde{y})=\widetilde{s}\left(\varphi^{\beta} \widetilde{u}\right)=u .
$$

I.e., $\widetilde{t}(\widetilde{x})=\widetilde{s}(\widetilde{y})$, namely, $(\widetilde{x}, \widetilde{y}) \in R_{2}$. Therefore,

$$
\operatorname{pr}_{12}(\widetilde{\Lambda}) \subseteq R_{2}
$$

Conversely, for any $(\widetilde{x}, \widetilde{y}) \in R_{2}$, assume that $\widetilde{t}(\widetilde{x})=\widetilde{s}(\widetilde{y})=u \in X_{0}$. Since $X_{1} \rightrightarrows X_{0}$ is $t$ connected and $\left(t_{\circ} \pi\right)(\widetilde{x})=u$, there exists a product $\varphi^{\alpha_{0}}$ of flows generated by vector fields of the form $\vec{V}$ for $V \in \Gamma(A)$, such that $\varphi^{\alpha_{0}}(u)=\pi(\widetilde{x})$. For each $V \in \Gamma(A)$, we denote the flow of the vector field $\widehat{\vec{V}} \in \mathfrak{X}\left(R_{1}\right)$ by $\Phi_{t}^{\alpha}$. Since $\pi_{*} \widehat{\vec{V}}=\vec{V}$, then

$$
\pi \circ \Phi_{t}^{\alpha}=\varphi_{t}^{\alpha_{0}} \circ \pi
$$

As each fibre of the $S^{1}$-bundle $R_{1} \rightarrow X_{1}$ is compact, $\Phi_{t}^{\alpha}$ is defined provided that $\varphi_{t}^{\alpha_{0}}$ is defined. Let $\Phi^{\alpha}$ denote the product of flows corresponding to $\varphi^{\alpha_{0}}$. Then we have

$$
\pi \circ \Phi^{\alpha}=\varphi^{\alpha_{0}} \circ \pi
$$

Hence

$$
\pi\left(\Phi^{\alpha}(\widetilde{u})\right)=\varphi^{\alpha_{0}}(u)=\pi(\widetilde{x}) .
$$

Therefore $\widetilde{x}=\lambda \cdot \Phi^{\alpha}(\widetilde{u})$ for some $\lambda \in S^{1}$. Note that the flow $\psi_{t}(\widetilde{x})=t \cdot \widetilde{x}$ on $R_{1}$ is generated by the standard Euler vector field $\xi$, which is also in $\mathcal{D}_{s}$. Hence we conclude that there exists a product of flows $\hat{\Phi}^{\alpha}$ generated by the vector fields in $\mathcal{D}_{s}$ such that $\widetilde{x}=\hat{\Phi}^{\alpha}(\widetilde{u})$. Similarly, we can find a product of flows $\hat{\Phi}^{\beta}$ generated by the vector fields in $\mathcal{D}_{t}$ such that $\widetilde{y}=\hat{\Phi}^{\beta}(\widetilde{u})$. So $\left(\widetilde{x}, \widetilde{y}, \hat{\Phi}^{\alpha} \hat{\Phi}^{\beta}(\widetilde{u})\right) \in \widetilde{\Lambda}$, i.e. $(\widetilde{x}, \widetilde{y})=\operatorname{pr}_{12}\left(\widetilde{x}, \widetilde{y}, \hat{\Phi}^{\alpha} \hat{\varphi}^{\beta}(\widetilde{u})\right) \in \operatorname{pr}_{12}(\widetilde{\Lambda})$. Thus we have proved that $\operatorname{pr}_{12}(\widetilde{\Lambda})=R_{2}$.

Finally, note that if $\widetilde{x}=\varphi^{\alpha} \widetilde{u}=\varphi_{1}^{\alpha} \widetilde{u}$ and $\widetilde{y}=\varphi^{\beta} \widetilde{u}$, then $\widetilde{z}=\varphi^{\alpha} \varphi^{\beta} \widetilde{u}$ and $z_{1}=\varphi_{1}^{\alpha} \varphi^{\beta} u$. Thus $\widetilde{z}=\varphi^{\beta} \varphi^{\alpha} \widetilde{u}=\varphi^{\beta}(\widetilde{x})=\widetilde{z}_{1}$. Similarly, one shows that $\widetilde{z}$ is also independent of the choice of the flows

$\varphi^{\beta}$. This shows that $\widetilde{\Lambda}$ is indeed a graph over $R_{2}$. Now the conclusion follows from Proposition 5.9 .

Remark It would be interesting to investigate how the integrability condition of CrainicFernandes [11] is related to the theorem above.

\section{References}

[1] Artin, M., Grothendieck, A., and Verdier, J.-L., Théorie des topos et cohomologie étale des schémas, Séminaire de géométrie algébrique du Bois-Marie (SGA4) Lecture Notes in Mathematics 269, 270, 305. Springer-Verlag, Berlin-New York, 1972-1973.

[2] Artin, M., and Mazur, B., Etale homotopy, Lecture Notes in Mathematics 100 Springer-Verlag, Berlin-New York 1969.

[3] Behrend, K., and Xu, P., $S^{1}$-bundles and gerbes over differentiable stacks, C. R. Acad. Sci. Paris, Serie I 336 (2003), 163-168. 
[4] Behrend, K., Xu, P. and Zhang, B., Equivariant gerbes over compact simple Lie groups. C. R. Math. Acad. Sci. Paris, Serie I 336 (2003), 251-256.

[5] Brylinski, J.-L., Loop spaces, characteristic classes and geometric quantization, Progress in Mathematics, 107 Birkhäuser, 1993.

[6] Brylinski, J.-L., Gerbes on complex reductive Lie groups, math.DG/0002158.

[7] Bunke, U., Schick, T., Spitzweck, M., Sheaf theory for stacks in manifolds and twisted cohomology for $S^{1}$-gerbes, Algebraic \& Geometric Topology 7 (2007), 1007-1062.

[8] Chatterjee, D., On the construction of abelian gerbes, $\mathrm{PhD}$ thesis (Cambridge), 1998.

[9] Coste, A., Dazord, P., and Weinstein, A, Groupoïdes symplectiques, Publications du Département Nouvelle Serie. A 2 1-62, Univ. Claude-Bernard, Lyon, (1987) 1-62.

[10] Crainic, M., Differentiable and algebroid cohomology, van Est isomorphisms, and characteristic classes, Comment. Math. Helv. 78 (2003), 681-721

[11] Crainic, M., and Fernandes, R., Integrability of Lie brackets, Ann. of Math. 157 (2003), $575-620$.

[12] Deligne, P., Théorie de Hodge. III, Inst. Hautes Études Sci. Publ. Math. 44 (1974), 5-77.

[13] Dijkgraaf, R., The mathematics of fivebranes, Documenta Math. Extra Volume ICM (1998).

[14] Dupont, J., Curvature and characteristic classes, Lecture Notes in Mathematics 640 SpringerVerlag, Berlin-New York, 1978.

[15] Duskin, J., An outline of nonabelian cohomology in a topos. I. The theory of bouquets and gerbes, Cahiers Topologie Géom. Différentielle23 (1982), 165-191.

[16] Freed, D., Higher algebraic structures and quantization, Commun. Math. Phys. 159 (1994), 343-398.

[17] Gawedzki, K., and Reis, N., WZW branes and gerbes, Rev. Math. Phys. 14 (2002), 1281-1334.

[18] Ginot, G., and Stienon, M., G-gerbes, principal 2-group bundles and characteristic classes, arXiv:0801.1238

[19] Giraud, J., Cohomologie non abélienne, Die Grundlehren der mathematischen Wissenschaften 179, Springer-Verlag 1971.

[20] Grothendieck, A., Revtements étales et groupe fondamental, Séminaire de géométrie algébrique du Bois-Marie 1960-1961 (SGA 1), Lecture Notes in Mathematics 224 SpringerVerlag, Berlin-New York, 1971.

[21] Hitchin, N., Lectures on special Lagrangian submanifolds, Winter School on Mirror Symmetry, Vector Bundles and Lagrangian Submanifolds (Cambridge, MA, 1999), AMS/IP Stud. Adv. Math. 23, 151-182.

[22] Hilsum, M., and Skandalis, G., Morphismes $K$-orientés d'espaces de feuilles et fonctorialité en théorie de Kasparov (d'après une conjecture d'A. Connes). Ann. Sci. École Norm. Sup. 20 (1987), 325-390.

[23] Kalkkinen, J., Gerbes of massive type II configurations, J. High Energy Physics 9907 (1999), $2-22$. 
[24] Kostant, B., Quantization and unitary representations. I. Prequantization. Lectures in modern analysis and applications, III, Lecture Notes in Math. 170 87-208.

[25] Laumon, G., and Moret-Bailly, L., Champs algébrique,, Results in Mathematics and Related Areas. 3rd Series. A Series of Modern Surveys in Mathematics, 39 Springer-Verlag, (2000).

[26] Laurent-Gengoux, C., Tu, J.-L., and Xu, P., Chern-Weil map for principal bundles over groupoids. Math Z. 255 (2007), 451-491.

[27] Lupercio, E., and Uribe, B., Gerbes over orbifolds and twisted K-theory, Comm. Math. Phys. 245 (2004), 449-489.

[28] Mackenzie, K., General theory of Lie groupoids and Lie algebroids, London Mathematical Society Lecture Note Series 213 Cambridge University Press, Cambridge, 2005.

[29] Meinrenken, E., The basic gerbe over a compact simple Lie group, Enseign. Math. (2)49 (2003), 307-333.

[30] Mikami, K., and Weinstein, A., Moments and reduction for symplectic groupoid actions, Publ. RIMS Kyoto Univ. 24 (1988), 121-140.

[31] Muhly, P., Renault, J. Williams, D., Equivalence and isomorphism for groupoid $C^{*}$-algebras, J. Operator Theory 17 (1987), 3-22.

[32] Murray, M. K., Bundle gerbes, J. London Math. Soc. 54 (1996), 403-416.

[33] Murray, M. K., Stevenson, D., Bundle gerbes: stable isomorphism and local theory, J. London Math. Soc. 62 (2000), 925-937.

[34] Metzler, D., Topological and smooth stacks, math.DG/0306176.

[35] Moerdijk, I., Orbifolds as groupoids: an introduction, Orbifolds in mathematics and physics (Madison, WI, 2001), Contemp. Math. 310, 205-222, 2002.

[36] Moerdijk, I. Lie groupoids, gerbes, and non-abelian cohomology, K-Theory 28 (2003), no. 3, $207-258$.

[37] Moerdijk, I., Introduction to the language of stacks and gerbes, math.AT/0212266.

[38] Moerdijk, I., Mrcun, Introduction to Foliations and Lie Groupoids, Cambridge Studies in Advanced Mathematics, 91, Cambridge University Press, Cambridge, 2003.

[39] Noohi, B., Fundamental groups of algebraic stacks, J. Inst. Math. Jussieu 3 (2004), 69-103.

[40] Noohi, B., Foundations of topological stacks I, math.AG/0503247.

[41] Stienon, M., Equivariant Dixmier-Douady classes, arXiv:0709.2720.

[42] Tu, J.-L., Groupoid cohomology and extensions, Trans. AMS 358 (2006), 4721-4747.

[43] Tu, J.-L., Xu, P., and Laurent-Gengoux, C., Twisted $K$-theory of differentiable stacks. Ann. Sci. École Norm. Sup. (4) 37 (2004), no. 6, 841-910.

[44] Tu, J.-L., and Xu, P., Chern character for twisted K-theory of orbifolds, Adv Math 207 (2006), $455-483$. 
[45] Tu, J.-L., and $\mathrm{Xu}, \mathrm{P}$., The ring structure for equivariant twisted K-theory, J. Reine Angew. Math. (to appear); math.KT/0604160.

[46] Sharpe, E., Discrete torsion, Phys. Rev. 68 (2003), 7-

[47] Souriau, J.-M., Structure of dynamical systems, A symplectic view of physics, Translated from the French by C. H. Cushman-de Vries, Progress in Mathematics 149 Birkhuser Boston, Inc., Boston, MA, 1997.

[48] Weil, A., Sur les théorèmes de de Rham, Comment. Math. Helv. 26, (1952). 119-145.

[49] Weinstein, A. and $\mathrm{Xu}, \mathrm{P}$. , Extensions of symplectic groupoids and quantization. J. Reine Angew. Math. 417 (1991), 159-189.

[50] Wirth, J., and Stasheff, J., Homotopy transition cocycles, math.AT/0609220. 